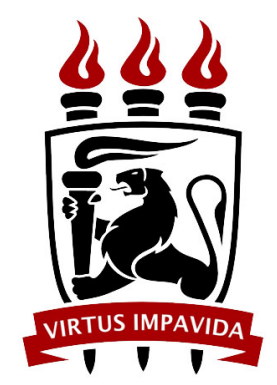

Universidade Federal de Pernambuco

Centro de Ciências Exatas e da Natureza

Programa de Pós-Graduação em Estatística

Inara Francoyse de Souza Pereira

\title{
Testing inference in heteroskedastic linear regressions: a comparison of two alternative approaches
}

Recife 
Inara Francoyse de Souza Pereira

Testing inference in heteroskedastic linear regressions: a comparison of two alternative approaches

Dissertação submetida como requerimento parcial para obtenção do grau de Mestre pelo Programa de Pós-Graduação em Estatística da Universidade Federal de Pernambuco.

Orientador: Francisco Cribari Neto

Recife

2018 


\section{P436t Pereira, Inara Francoyse de Souza}

Testing inference in heteroskedastic linear regressions: a comparison of two alternative approaches / Inara Francoyse de Souza Pereira. - 2018.

68 f.: il., fig., tab.

Orientador: Francisco Cribari Neto.

Dissertação (Mestrado) - Universidade Federal de Pernambuco. CCEN, Estatística, Recife, 2018.

Inclui referências e apêndices.

1. Estatística. 2. Regressão linear. I. Cribari Neto, Francisco (orientador). II. Título.

310

CDD (23. ed.)

UFPE- MEI 2018-058 
INARA FRANCOYSE DE SOUZA PEREIRA

TESTING INFERENCE IN HETEROSKEDASTIC LINEAR REGRESSIONS: A COMPARISON OF TWO ALTERNATIVE APPROACHES

Dissertação apresentada ao Programa de Pós-Graduação em Estatística da Universidade Federal de Pernambuco, como requisito parcial para a obtenção do título de Mestre em Estatística.

Aprovada em: 20 de fevereiro de 2018.

\title{
BANCA EXAMINADORA
}

\section{Prof. Francisco Cribari Neto} UFPE

\author{
Prof. ${ }^{\text {a }}$ Fernanda de Bastiani \\ UFPE
}
Prof. ${ }^{\mathrm{a}}$ Tatiene Correia de Souza UFPB




\section{Acknowledgement}

I would like to thank my family, especially my parents, Inelda and Francinaldo, my grandparents, Valtomiro and Socorro, my brothers, Ikaro and Ryan, my nephews, Isis and Pietro, my godson, Vicente, my uncles, Neto and Rubhia, my cousins, Victor, Vanessa, Sofia and Vinícius and all my friends for their affection, love, encouragement, concern and support in difficult times.

I also express gratitude to my fiancée, Jhonnata, who help me when I was still getting used to living in Recife. Thank you for your love, affection, patience and dedication.

I thank my adviser, Francisco Cribari Neto, for his dedication and patience during the preparation of this dissertation and also for sharing his knowledge with me. I thank him for his wise words, for trusting my ability to overcome difficulties and for his substantial contribution to task of writing this manuscript in English.

I also thank the dissertation committee members for reviewing my work and for their suggestions and constructive criticism.

A big thank you to Valéria Bittencourt, the Graduate Program in Statistics chief secretary, for her competent work, affection and dedication.

Finally, I would like to the financial support I received from CAPES during the past two years. 
A educação tem raízes amargas, mas os seus frutos são doces.

Aristóteles 


\section{Abstract}

We consider the issue of performing testing inferences on the parameters that index the linear regression model under heteroskedasticity of unknown form. Quasi- $t$ test statistics use asymptotically correct standard errors obtained from heteroskedasticity-consistent covariance matrix estimators. An alternative approach involves making an assumption about the functional form of the response variances and jointly modeling mean and dispersion effects. In this dissertation we compare the accuracy of testing inferences made using the two approaches. We consider several different quasi- $t$ tests and also $z$ tests performed after generalized least squares estimation which was carried out using three different estimation strategies. Our numerical evaluations were performed using different models, different sample sizes, and different heteroskedasticity strengths. The numerical evidence shows that some quasi- $t$ tests are considerably less size distorted in small samples than the tests carried out after the jointly modeling mean and dispersion effects. Finally, we present and discuss two empirical applications.

Keywords: Generalized least squares. Heteroskedasticity. Linear regression. Ordinary least squares. Quasi- $t$ test. $z$ test. 


\section{Resumo}

Na presente dissertação nós consideramos a realização de inferências por teste de hipótese sobre os parâmetros que indexam o modelo linear de regressão sob heteroscedasticidade de forma desconhecida. As estatísticas de teste quasi- $t$ empregam erros-padrão assintoticamente corretos oriundos de estimadores consistentes da matriz de covariância do estimador de mínimos quadrados ordinários dos parâmetros de regressão. Um enfoque alternativo envolve a modelagem das variâncias das respostas, ou seja, a modelagem conjunta de efeitos médios e de dispersão. Nós comparamos os dois enfoques através de várias simulações de Monte Carlo. Consideramos vários testes quasi- $t$ e testes $z$ realizados após estimação por mínimos quadrados generalizados realizada através de três enfoques distintos. Nossas avaliações numéricas foram realizadas utilizando diferentes modelos, tamanhos de amostra e graus de heteroscedasticidade. A evidência numérica indica que os testes quasi- $t$ tendem a apresentar distorções de tamanho consideravelmente menores em pequenas amostras do que os testes realizados após a modelagem conjunta dos efeitos médios e de dispersão. Por fim, apresentamos e discutimos duas aplicações empíricas.

Palavras-chave: Mínimos quadrados generalizados. Heteroscedasticidade. Regressão linear. Mínimos quadrados ordinários. Teste quasi- $t$. Teste $z$. 


\section{List of Figures}

5.1 Per capita spending on public schools vs. per capita income $(\times 10,000)$. 


\section{List of Tables}

4.1 Null rejection rates (\%), first simulation experiment; $\lambda \approx 51$.

4.2 Null rejection rates (\%), first simulation experiment; $\lambda \approx 101$. 26

4.3 Null rejection rates $(\%)$, second simulation experiment; $\lambda \approx 6$. 28

4.4 Null rejection rates (\%), second simulation experiment; $\lambda \approx 26$. 29

4.5 Null rejection rates (\%), third simulation experiment; homoskedasticity and heteroskedasticity.

4.6 Nonnull rejection rates (\%), first simulation experiment; $\lambda \approx 51$. 31

4.7 Nonnull rejection rates (\%), first simulation experiment; $\lambda \approx 101$.

4.8 Nonnull rejection rates (\%), second simulation experiment; $\lambda \approx 6$. 33

4.9 Nonnull rejection rates (\%), second simulation experiment; $\lambda \approx 26$. 34

4.10 Nonnull rejection rates $(\%)$, third simulation experiment; homoskedasticity and heteroskedasticity.

4.11 True variances of $\hat{\beta}_{2}$ and $\tilde{\beta}_{2}$. 36

4.12 Mean estimated variances of $\hat{\beta}_{2}$ and $\tilde{\beta}_{2}$. 36

5.1 Descriptive Statistics, complete data; man-hours for manning installations data. 37

5.2 Standard errors, complete data; man-hours for manning installations data. $\quad 38$

5.3 Tests $p$-values; man-hours for manning installations data. 39

5.4 Parameter estimates, incomplete data; man-hours for manning installations data. 39

5.5 Standard errors, incomplete data; man-hours for manning installations data. 39

5.6 Descriptive Statistics, complete data; per capita spending on public education data. $\quad 40$

5.7 Standard errors, complete data; per capita spending on public education data. 41

5.8 Tests $p$-values; per capita spending on public education data. 42

5.9 Parameter estimates, incomplete data; per capita spending on public education data.

5.10 Standard errors, incomplete data; per capita spending on public education data. $\quad 42$

5.11 Tests $p$-values; per capita spending on public education data; alternative skedastic function. 


\section{Contents}

1 Introduction. . . . . . . . . . . . . . . . 11

1.1 Initial considerations. . . . . . . . . . . . . . . . . . . . . 11

1.2 Organization of dissertation . . . . . . . . . . . . . . . . . . . . . . . . . . . . . 12

1.3 Computing platforms . . . . . . . . . . . . . . . . . . . . . . . . . . . 13

2 Model and estimators . . . . . . . . . . . . . . . . . . 14

3 Estimated generalized least squares . . . . . . . . . . . . 19

4 Numerical evaluation . . . . . . . . . . . . . . . . 22

4.1 Simulation setup . . . . . . . . . . . . . . . . . . . . . . . . . . . . . . 22

4.2 Size simulations . . . . . . . . . . . . . . . . . . . . . . . . . . 24

4.3 Power simulations. . . . . . . . . . . . . . . . . . . . . . . . . . . . . . . . 27

4.4 Variability . . . . . . . . . . . . . . . . . . . . . . . . 30

5 Empirical applications . . . . . . . . . . . . . 37

5.1 Man-hours for manning installations data. . . . . . . . . . . . . . . . . 37

5.2 Per capita spending on public education . . . . . . . . . . . . . . . . . . . . . . 39

6 Concluding remarks ................ . . 44

References ................... . . 46

Appendix A - Size simulation. . . . . . . . . . . . . 48

Appendix B - Power simulation. . . . . . . . . . . . 58 


\section{Introduction}

\subsection{Initial considerations}

The linear regression model is commonly used in empirical analyses in a variety of fields. It assumes that the mean of the variable of interest (response, dependent variable) equals a linear predictor that involves regression coefficients and a set of independent variables (covariates, regressors). Alternatively, the response is taken to be the sum of the linear predictor (systematic component) and a random, unobservable error. Parameter estimation is typically carried out by ordinary least squares (which requires no distributional assumptions) from a sample of $n$ observations. It is oftentimes assumed that all $n$ errors (or, equivalently, that all $n$ responses) share the same variance. Such an assumption is known as homoskedasticity. Under constant error variances and normality, exact testing inferences can be carried out using standard $t$ and $F$ tests. The homoskedasticity assumption, nonetheless, is commonly violated when working with cross-sectional datasets. When that happens, the ordinary least square estimator of the vector of regression coefficients remains unbiased, consistent, and asymptotically normally distributed. Its usual covariance matrix estimator, however, becomes biased and is not consistent under unequal error variances. The common practice is to use a heteroskedasticity-consistent covariance matrix estimator, i.e., an estimator for the variance of the vector of ordinary least regression coefficients estimators that is consistent under both homoskedasticity and heteroskedasticity of unknown form. We can then obtain asymptotically correct standard errors and use them to construct quasi- $t$ test statistics that are, under the null hypothesis, asymptotically distributed as standard normal. Hence, without the need to assume that the errors are normally distributed, the practitioner can perform testing inference on the regression parameters.

An alternative approach is to jointly model the response mean and variance, and then perform $z$ tests. Parameter estimation is performed by estimated (feasible) generalized least squares, i.e., using generalized least squares and replacing the unknown variances with the corresponding estimates. This approach entails the extra burden of modeling dispersion effects in addition to mean effects. The most commonly model assumes that heteroskedasticity is multiplicative, as proposed by Harvey (1976). The multiplicative functional form guarantees that all variance estimates are positive.

The two testing strategies listed above use standard errors obtained from different covariance matrix estimators: quasi- $t$ test statistics use standard errors obtained from a heteroskedasticityrobust covariance matrix estimators whereas in the $z$ test the standard error is obtained from an estimate of the estimated generalized least squares covariance matrix. 
Atkinson, Riani, and Torti (2016) have recently compared the covariance matrix estimators obtained using the two approaches. They show that covariance matrix estimation is more accurate when performed via estimated generalized least squares even when the skedastic function is misspecified. Their focus lies in the accuracy of variance and covariance estimates. According to Simonoff (1993), nonetheless, since estimated variances are used primarily for performing inferences, comparisons involving different variance estimators should be related to their intended use, such as the empirical coverages of associated confidence intervals and the true nominal sizes of associated tests. This is the approach we shall pursue in this dissertation. In what follows, we shall address the following question: Are $z$ testing inferences made after estimated generalized least squares estimation more accurate than those based on quasi- $t$ tests? An appealing feature of the latter is that it does not require assumptions about the skedastic function.

In order to motivate the analysis in the remainder of our dissertation, consider the data analyzed by Cribari-Neto (2004). The variable of interest ( $y$ ) is per capita spending on public schools in the U.S. (49 states and Washington, D.C.; sample size: 50 observations). It is expected that such per capita spending grow with per capita income $(x)$, i.e., richer states are expected to spend more on public schools than less developed states on a per capita basis. We postulate two different relationships, namely: quadratic and linear. In order to distinguish between them, we write the model as $y_{i}=\beta_{1}+\beta_{2} x_{i}+\beta_{3} x_{i}^{2}+\epsilon_{i}, i=1, \ldots, 50$, where $\epsilon_{i}$ is a zero mean random error, and test $\mathcal{H}_{0}: \beta_{3}=0$ (linear) against $\mathcal{H}_{1}: \beta_{3} \neq 0$ (quadratic). Inspection of the data indicates that there is a very atypical data point, namely: Alaska. Additionally, there appears to be heteroskedasticity. A practitioner may be interested in knowing whether the best testing strategy involves the use of a quasi- $t$ test, whose test statistic is based on a heteroskedasticityrobust standard error, or the use of a $z$ test carried out after estimating the model parameters by estimated generalized least squares. Notice that the latter approach requires one to model not only mean effects but also dispersion effects. Interestingly, the two approaches (i.e., the $z$ test and the best performing quasi- $t$ tests) may yield different inferences at the $10 \%$ significance level. It will also be seen that $z$ testing inferences are heavily dependent on how the dispersion effects are modeled. We shall return to this application in Chapter 5.

\subsection{Organization of dissertation}

The dissertation unfolds as follows. There are six chapters, including this introduction. In Chapter 2 we briefly present the linear regression model and the heteroskedasticity-consistent covariance matrix estimators that will be used in the following chapters. Chapter 3 is devoted to estimated generalized least squares estimation of the parameters that index the linear regression model. The numerical evidence on the finite sample performances of the different tests are presented in Chapter 4. We report results on the sizes and powers of the tests, i.e., we examine their performances under both the null and alternative hypotheses. The data generating processes we consider include heteroskedastic and homoskedastic model structures, balanced and leveraged data, and also normal and nonnormal random errors. In Chapter 5 we present and discuss two empirical applications. One of them is the application briefly introduced above. Finally, some concluding remarks are offered in Chapter 6. 


\subsection{Computing platforms}

The simulations were carried out using the Ox matrix programming language (version 7.2). The Ox programming language is an object-oriented matrix programming language developed by Jurgen Doornik and it is freely available for academic usage at http://www . doornik . com. The appendix of this dissertation contains two programs written in Ox that were used to obtain some of the numerical results we present. In particular, it contains Ox code for a size simulation and for a power simulation. For more information about the Ox matrix programming language, see Doornik (2009). The figure was produced using the $\mathrm{R}$ statistical computing environment (version 3.2.4). $\mathrm{R}$ is a free software environment for statistical computing and graphics. For details, see R Core Team (2016). The typesetting environment chosen was LATEX (Lamport 1986). $\mathrm{LT}_{\mathrm{E}} \mathrm{X}$ is a document preparation system developed based on $\mathrm{T}_{\mathrm{E}} \mathrm{X}$, which was developed by Donald Knuth. It is freely available. 


\section{Model and estimators}

The linear regression model can be written as

$$
y=X \beta+\epsilon,
$$

where $\boldsymbol{y}$ is an $n \times 1$ vector of responses (observations on the dependent variable), $X$ is an $n \times p$ matrix of observations on $p$ covariates $(\operatorname{rank}(X)=p<n), \boldsymbol{\beta}=\left(\beta_{1}, \ldots, \beta_{p}\right)^{\prime}$ is a $p$-vector of unknown regression parameters and $\epsilon=\left(\epsilon_{1}, \ldots, \epsilon_{n}\right)^{\prime}$ is an $n$-vector of random errors.

The following assumptions are usually made:

A1 Model (2.1) is correctly specified;

A2 $\mathbb{E}\left(\epsilon_{i}\right)=0, i=1, \ldots, n ;$

A3 $\operatorname{var}\left(\epsilon_{i}\right)=\mathbb{E}\left(\epsilon_{i}^{2}\right)=\sigma^{2} \forall i\left(0<\sigma^{2}<\infty\right) ;$

A4 $\mathbb{E}\left(\epsilon_{i} \epsilon_{j}\right)=0 \forall i \neq j$

A5 $\lim _{n \rightarrow \infty} n^{-1} X^{\prime} X=Q$, where $Q$ is a positive definite matrix.

The ordinary least squares estimator (OLSE) of $\boldsymbol{\beta}$ is obtained by minimizing the sum of squared errors:

$$
\boldsymbol{\epsilon}^{\prime} \boldsymbol{\epsilon}=(\boldsymbol{y}-X \boldsymbol{\beta})^{\prime}(\boldsymbol{y}-X \boldsymbol{\beta}) .
$$

It can be expressed in closed-form as

$$
\begin{aligned}
\hat{\boldsymbol{\beta}} & =\left(X^{\prime} X\right)^{-1} X^{\prime} \boldsymbol{y} \\
& =\left(X^{\prime} X\right)^{-1} X^{\prime}(X \boldsymbol{\beta}+\boldsymbol{\epsilon}) \\
& =\left(X^{\prime} X\right)^{-1} X^{\prime} X \boldsymbol{\beta}+\left(X^{\prime} X\right)^{-1} X^{\prime} \boldsymbol{\epsilon} \\
& =\boldsymbol{\beta}+\left(X^{\prime} X\right)^{-1} X^{\prime} \boldsymbol{\epsilon} .
\end{aligned}
$$

Under Assumptions A1 and A2, $\hat{\boldsymbol{\beta}}$ is unbiased for $\boldsymbol{\beta}, \mathbb{E}(\hat{\boldsymbol{\beta}})=\boldsymbol{\beta} \forall \boldsymbol{\beta} \in \mathbb{R}^{k}$ as we can see below:

$$
\begin{aligned}
\mathbb{E}(\hat{\boldsymbol{\beta}}) & =\mathbb{E}\left[\boldsymbol{\beta}+\left(X^{\prime} X\right)^{-1} X^{\prime} \boldsymbol{\epsilon}\right] \\
& =\boldsymbol{\beta}+\left(X^{\prime} X\right)^{-1} X^{\prime} \mathbb{E}(\boldsymbol{\epsilon}) \\
& =\boldsymbol{\beta} .
\end{aligned}
$$


It is important to note that it is not necessary to assume homoskedasticity to establish the unbiasedness of the OLSE.

Under Assumptions A1, A2 and A4, the covariance matrix of $\epsilon$ is $\Phi=\operatorname{diag}\left\{\sigma_{1}^{2}, \ldots, \sigma_{n}^{2}\right\}$. It then follows that under homoskedasticity (Assumption A3), $\Phi=\sigma^{2} I_{n}$, where $I_{n}$ is the $n \times n$ identity matrix. Under Assumptions A1, A 2 and A4, the covariance matrix of $\hat{\boldsymbol{\beta}}$ can be expressed as

$$
\begin{aligned}
\Psi & =\mathbb{E}\left[(\hat{\boldsymbol{\beta}}-\boldsymbol{\beta})(\hat{\boldsymbol{\beta}}-\boldsymbol{\beta})^{\prime}\right] \\
& =\mathbb{E}\left[\left(\boldsymbol{\beta}+\left(X^{\prime} X\right)^{-1} X^{\prime} \boldsymbol{\epsilon}-\boldsymbol{\beta}\right)\left(\boldsymbol{\beta}+\left(X^{\prime} X\right)^{-1} X^{\prime} \boldsymbol{\epsilon}-\boldsymbol{\beta}\right)^{\prime}\right] \\
& =\mathbb{E}\left[\left(X^{\prime} X\right)^{-1} X^{\prime} \boldsymbol{\epsilon} \boldsymbol{\epsilon}^{\prime} X\left(X^{\prime} X\right)^{-1}\right] \\
& =\left(X^{\prime} X\right)^{-1} X^{\prime} \Phi X\left(X^{\prime} X\right)^{-1} .
\end{aligned}
$$

$\Phi$ is the error covariance matrix, which can also be written as $\sigma^{2} \Omega$, where $\sigma^{2}$ is a positive scalar. When Assumption A3 holds (i.e., under homoskedasticity), $\Phi=\sigma^{2} I_{n}$ and then $\Psi=\sigma^{2}\left(X^{\prime} X\right)^{-1}$, which can be easily estimated as $\hat{\sigma}^{2}\left(X^{\prime} X\right)^{-1}$, where $\hat{\sigma}^{2}=\hat{\boldsymbol{\epsilon}}^{\prime} \hat{\boldsymbol{\epsilon}} /(n-p)$ and $\hat{\boldsymbol{\epsilon}}=\boldsymbol{y}-X \hat{\boldsymbol{\beta}}$ are the residuals. Under Assumptions A1 through A4, $\hat{\boldsymbol{\beta}}$ is the best linear unbiased estimator (BLUE) of $\boldsymbol{\beta}$ (Gauss-Markov Theorem). This result implies that under homoskedasticity the OLSE has smaller variability than any other estimator of $\boldsymbol{\beta}$ that is both linear and unbiased. Under A1, $\mathrm{A} 2$ and A5 (thus, without assuming homoskedasticity), $\hat{\boldsymbol{\beta}}$ is consistent for $\boldsymbol{\beta}$, i.e., $\hat{\boldsymbol{\beta}}$ converges in probability to $\boldsymbol{\beta}$, which will be denoted by $\operatorname{pim}(\hat{\boldsymbol{\beta}})=\boldsymbol{\beta}$, where plim denotes probability limit. In order to prove this result, we use the fact that if $g(\cdot)$ is a continuous function and $z_{n}$ is a sequence of random vectors, then

$$
\operatorname{plim}\left(g\left(z_{n}\right)\right)=g\left(\operatorname{plim}\left(z_{n}\right)\right),
$$

provided that $\operatorname{plim}\left(z_{n}\right)$ exists. Under the Assumptions A1 and A5, we have

$$
\begin{aligned}
\operatorname{plim}(\hat{\boldsymbol{\beta}}) & =\operatorname{plim}\left(\boldsymbol{\beta}+\left(X^{\prime} X\right)^{-1} X^{\prime} \boldsymbol{\epsilon}\right) \\
& =\boldsymbol{\beta}+\operatorname{plim}\left(X^{\prime} X\right)^{-1} \operatorname{plim}\left(X^{\prime} \boldsymbol{\epsilon}\right) \\
& =\boldsymbol{\beta}+\operatorname{plim}\left(n^{-1} X^{\prime} X\right)^{-1} \operatorname{plim}\left(n^{-1} X^{\prime} \boldsymbol{\epsilon}\right) \\
& =\boldsymbol{\beta}+\left(\operatorname{plim} n^{-1} X^{\prime} X\right)^{-1} \operatorname{plim}\left(n^{-1} X^{\prime} \boldsymbol{\epsilon}\right) \\
& =\boldsymbol{\beta}+Q^{-1} \operatorname{plim}\left(n^{-1} X^{\prime} \boldsymbol{\epsilon}\right) .
\end{aligned}
$$

Note that $X^{\prime} \boldsymbol{\epsilon}=\sum_{i=1}^{n} \boldsymbol{x}_{i} \epsilon_{i}$, where $\boldsymbol{x}_{i}$ is the $i$ th line of $X$ (as a column vector). It follows from the Law of Large Numbers that $n^{-1} X^{\prime} \epsilon$ converges in probability to $\mathbb{E}\left(\boldsymbol{x}_{i} \epsilon_{i}\right)$, which by Assumption A2, equals 0 . That is, $\operatorname{plim}\left(n^{-1} X^{\prime} \epsilon\right)=0$. Therefore,

$$
\operatorname{plim}(\hat{\boldsymbol{\beta}})=\boldsymbol{\beta} .
$$

It is important to emphasize that this result holds regardless of whether Assumption A3 is satisfied. When Assumption A3 or Assumption A4 is not valid, the OLSE of $\boldsymbol{\beta}$ is no longer efficient.

When the errors are heteroskedastic but $\Phi$ is known (which rarely happens), one can use the generalized least squares estimator (GLSE), which is given by $\hat{\boldsymbol{\beta}}_{G}=\left(X^{\prime} \Phi^{-1} X\right)^{-1} X^{\prime} \Phi^{-1} \boldsymbol{y}$. Its covariance matrix is $\operatorname{cov}\left(\hat{\boldsymbol{\beta}}_{G}\right)=\sigma^{2}\left(X^{\prime} \Omega^{-1} X\right)^{-1}$, as shown below. 
The initial idea is to transform the model given in (2.1) using $P$, a matrix of dimension $n \times n$ which satisfies

$$
P \Omega P^{\prime}=I_{n} .
$$

Since $\Omega$ is positive-definite, $P$ always exists. Notice that

$$
\Omega^{-1}=P^{\prime} P .
$$

We can use $P$ to transform Model (2.1) as follows:

$$
P \boldsymbol{y}=P X \boldsymbol{\beta}+P \epsilon .
$$

That is, we have

$$
\boldsymbol{y}^{*}=X^{*} \boldsymbol{\beta}+\boldsymbol{\epsilon}^{*},
$$

where $\boldsymbol{y}^{*}=P \boldsymbol{y}, X^{*}=P X$ and $\boldsymbol{\epsilon}^{*}=P \boldsymbol{\epsilon}$. The vector of transformed errors $\boldsymbol{\epsilon}^{*}$ has mean zero:

$$
\mathbb{E}\left(\epsilon^{*}\right)=\mathbb{E}(P \boldsymbol{\epsilon})=P \mathbb{E}(\boldsymbol{\epsilon})=0 .
$$

That is, Assumption A2 is satisfied. The covariance matrix of the vector of transformed erros can be easily obtained:

$$
\mathbb{E}\left(\epsilon^{*} \epsilon^{*^{\prime}}\right)=\mathbb{E}\left(P \boldsymbol{\epsilon} \epsilon^{\prime} P^{\prime}\right)=P \mathbb{E}\left(\boldsymbol{\epsilon} \epsilon^{\prime}\right) P^{\prime}=\sigma^{2} P \Omega P^{\prime}=\sigma^{2} I_{n}
$$

Thus, Assumptions A2 through A4 hold for the transformed model. Minimization of the sum of squared errors yields the following estimator of $\boldsymbol{\beta}$ :

$$
\begin{aligned}
\hat{\boldsymbol{\beta}}_{G} & =\left(X^{*^{\prime}} X^{*}\right)^{-1} X^{*^{\prime}} \boldsymbol{y}^{*} \\
& =\left(X^{\prime} P^{\prime} P X\right)^{-1} X^{\prime} P^{\prime} P \boldsymbol{y} \\
& =\left(X^{\prime} \Omega^{-1} X\right)^{-1} X^{\prime} \Omega^{-1} \boldsymbol{y} \\
& =\left(X^{\prime} \sigma^{-2} \Omega^{-1} X\right)^{-1} X^{\prime} \sigma^{-2} \Omega^{-1} \boldsymbol{y} \\
& =\left(X^{\prime} \Phi^{-1} X\right)^{-1} X^{\prime} \Phi^{-1} \boldsymbol{y} .
\end{aligned}
$$

Note that $\hat{\boldsymbol{\beta}}_{G}$ is unbiased for $\boldsymbol{\beta}$ :

$$
\begin{aligned}
\mathbb{E}\left(\hat{\boldsymbol{\beta}}_{G}\right) & =\left(X^{\prime} \Phi^{-1} X\right)^{-1} X^{\prime} \Phi^{-1} \mathbb{E}(\boldsymbol{y}) \\
& =\left(X^{\prime} \Phi^{-1} X\right)^{-1} X^{\prime} \Phi^{-1} X \boldsymbol{\beta} \\
& =\boldsymbol{\beta}
\end{aligned}
$$

The covariance matrix of $\hat{\boldsymbol{\beta}}_{G}$ is

$$
\operatorname{cov}\left(\hat{\boldsymbol{\beta}}_{G}\right)=\sigma^{2}\left(X^{*^{\prime}} X^{*}\right)^{-1}=\left(X^{\prime} \Phi^{-1} X\right)^{-1}=\sigma^{2}\left(X^{\prime} \Omega^{-1} X\right)^{-1} .
$$

The GLSE is efficient in the sense of the Gauss-Markov Theorem. 
Since $\Phi$ is typically unknown, $\hat{\boldsymbol{\beta}}_{G}$ cannot be computed. A possible solution is to assume a functional form for the $n$ variances, and then estimate $\Phi$. The estimated (or feasible) generalized least squares (EGLS) estimator is obtained by replacing $\Phi$ by such an estimate. It is given by

$$
\hat{\hat{\boldsymbol{\beta}}}=\left(X^{\prime} \hat{\Phi}^{-1} X\right)^{-1} X^{\prime} \hat{\Phi}^{-1} \boldsymbol{y} .
$$

Direct estimation of $\Phi$ is problematic, since this matrix contains $n$ unknown variances. A possible solution is to postulate a model for the variances, and then estimate the parameters of such a model. The most commonly used model for the error variances is the multiplicative model proposed by Harvey (1976). See the next chapter for further details.

A more commonly used approach involves basing inferences on $\hat{\boldsymbol{\beta}}$ coupled with a consistent estimator for its covariance matrix, i.e., with a covariance matrix estimator that is consistent under both homoskedasticity and heteroskedasticity of unknown form. The most well known consistent covariance estimator is known as HCO and was introduced by White (1980):

$$
\mathrm{HC} 0=\left(X^{\prime} X\right)^{-1} X^{\prime} \hat{\Phi}_{0} X\left(X^{\prime} X\right)^{-1},
$$

where $\hat{\Phi}_{0}=\operatorname{diag}\left\{\hat{\epsilon}_{1}^{2}, \ldots, \hat{\epsilon}_{n}^{2}\right\}$.

It has been shown that $\mathrm{HCO}$ can be considerably biased in finite samples, especially when the data contain high leverage data points; see, e.g., Chesher and Jewitt (1987). In particular, it tends to 'optimistic', i.e., it tends to underestimate the true variances. As a consequence, quasi- $t$ tests whose statistic use $\mathrm{HCO}$ standard errors tend to be liberal, i.e., such tests tend to overreject the null hypothesis when such a hypothesis is true.

MacKinnon and White (1985) proposed two alternative covariance matrix estimators. They both include finite sample corrections. When all errors share the same variance, it can be shown that

$$
\mathbb{E}\left(\hat{\epsilon}_{i}^{2}\right)=\left(1-h_{i}\right) \sigma^{2},
$$

where $h_{i}$ is the $i$ th diagonal element of $H=X\left(X^{\prime} X\right)^{-1} X^{\prime}$, the 'hat matrix'. It is important to note the $h_{i}$ 's are the leverage measures of the different observations. Building up on previous results by Horn, Horn, and Duncan (1975), MacKinnon and White (1985) introduced the estimator known as HC2. It is given by

$$
\mathrm{HC} 2=\left(X^{\prime} X\right)^{-1} X^{\prime} \hat{\Phi}_{2} X\left(X^{\prime} X\right)^{-1},
$$

where $\hat{\Phi}_{2}=\operatorname{diag}\left\{\hat{\epsilon}_{1}^{2} /\left(1-h_{1}\right), \ldots, \hat{\epsilon}_{n}^{2} /\left(1-h_{n}\right)\right\}$. Using (2.2) it is possible to show that HC2 is unbiased under homoskedasticity.

The authors have also introduced a jackknife covariance matrix estimator. It can be approximated by the following estimator, which is known as HC3:

$$
\mathrm{HC} 3=\left(X^{\prime} X\right)^{-1} X^{\prime} \hat{\Phi}_{3} X\left(X^{\prime} X\right)^{-1},
$$

where $\hat{\Phi}_{3}=\operatorname{diag}\left\{\hat{\epsilon}_{1}^{2} /\left(1-h_{1}\right)^{2}, \ldots, \hat{\epsilon}_{n}^{2} /\left(1-h_{n}\right)^{2}\right\}$.

The HC4 heteroskedasticity-consistent covariance matrix estimator was proposed by CribariNeto (2004). It is given by

$$
\mathrm{HC} 4=\left(X^{\prime} X\right)^{-1} X^{\prime} \hat{\Phi}_{4} X\left(X^{\prime} X\right)^{-1},
$$


where $\hat{\Phi}_{4}=\operatorname{diag}\left\{\hat{\epsilon}_{1}^{2} /\left(1-h_{1}\right)^{\delta_{1}}, \ldots, \hat{\epsilon}_{n}^{2} /\left(1-h_{n}\right)^{\delta_{n}}\right\}, \delta_{i}=\min \left\{4, h_{i} / \bar{h}\right\}$ and $\bar{h}=n^{-1} \sum_{i=1}^{n} h_{i}$, i.e., $\bar{h}$ is the mean leverage. Notice that the exponent of $\left(1-h_{i}\right)$ is not constant; it equals the ratio between the $i$ th leverage measure and the mean leverage up to a truncation constant.

A variant of the HC4 estimator was introduced by Cribari-Neto and Silva (2011). It is known as $\mathrm{HC} 4 \mathrm{~m}$ and is given by

$$
\mathrm{HC} 4 \mathrm{~m}=\left(X^{\prime} X\right)^{-1} X^{\prime} \hat{\Phi}_{4 m} X\left(X^{\prime} X\right)^{-1},
$$

where $\hat{\Phi}_{4 m}=\operatorname{diag}\left\{\hat{\epsilon}_{1}^{2} /\left(1-h_{1}\right)^{\theta_{1}}, \ldots, \hat{\epsilon}_{n}^{2} /\left(1-h_{n}\right)^{\theta_{n}}\right\}$. Here, $\theta_{i}=\min \left\{v_{1}, h_{i} / \bar{h}\right\}+\min \left\{v_{2}, h_{i} / \bar{h}\right\}$, where $v_{1}$ and $v_{2}$ are positive real constants. Based on numerical evidence, the authors proposed using $v_{1}=1.0$ and $v_{2}=1.5$. For details, see Cribari-Neto and Silva (2011).

The above heteroskedasticity-robust covariance matrix estimators can be used for interval estimation and testing inferences. Heteroskedasticity-robust interval estimation was addressed by Cribari-Neto and Lima (2009). Heteroskedasticity-robust testing inferences were considered by several authors; see, e.g., Cribari-Neto and Lima (2010) and Long and Ervin (2000). 


\section{Estimated generalized least squares}

As noted in the previous chapter, an alternative approach involves modeling the error variances. Consider the following linear model:

$$
y_{i}=\boldsymbol{x}_{i}^{\prime} \boldsymbol{\beta}+\sigma_{i} \epsilon_{i}, \quad i=1, \ldots, n,
$$

where $\boldsymbol{x}_{i}$ is a $p \times 1$ vector of observations on the independent variables and the errors are assumed to be standard normally distributed. Following Harvey (1976), the $i$ th error variance is modeled as

$$
\sigma_{i}^{2}=\operatorname{var}\left(y_{i}\right)=\exp \left\{\boldsymbol{z}_{i}^{\prime} \gamma\right\}=\exp \left\{\gamma_{1}+\gamma_{2} z_{i 2}+\cdots+\gamma_{s} z_{i s}\right\}
$$

where $\gamma$ is an $s \times 1$ vector of parameters and $z_{i}^{\prime}$ denotes the $i$ th row of $Z$, an $n \times s$ matrix that contains observations on the dispersion covariates which are usually, though not necessarily, related to the mean regressors. This model is known as the multiplicative heteroskedastic linear regression model.

The above model was recently revisited by Atkinson, Riani, and Torti (2016). For a given $\boldsymbol{\gamma}$, they estimate of $\boldsymbol{\beta}$ by weighted least squares. The weights used in the estimation of $\boldsymbol{\beta}$ are specified as

$$
w_{i}=1 / \sigma_{i}^{2} .
$$

The estimator of $\boldsymbol{\beta}$ can thus be written as

$$
\tilde{\boldsymbol{\beta}}=\left(X^{\prime} W X\right)^{-1} X^{\prime} W \boldsymbol{y},
$$

where $W=\operatorname{diag}\left\{w_{1}, \ldots, w_{n}\right\}$ is the $n \times n$ weight matrix. Alternatively, $\tilde{\boldsymbol{\beta}}$ can be computed by regressing $W^{1 / 2} \boldsymbol{y}$ on $W^{1 / 2} X$ using ordinary least squares. Finally, $\gamma$ can be estimated by maximum likelihood making use of the normality assumption.

Notice that the $i$ th weight is now given by $\tilde{w}_{i}=1 / \tilde{\sigma}_{i}^{2}$, where $\tilde{\sigma}_{i}^{2}=\exp \left(z_{i}^{\prime} \tilde{\gamma}\right)$. Let $\tilde{W}$ be the $n$-dimensional diagonal matrix that contains the estimated weights. The estimated covariance matrix of $\tilde{\boldsymbol{\beta}}$ is $\left(X^{\prime} \tilde{W} X\right)^{-1}$.

For a given $\beta$, the parameter vector $\gamma$ can be estimated by maximizing the following loglikelihood function:

$$
\begin{aligned}
\ell(\gamma) & =\log \left\{\prod_{i=1}^{n}\left[\left(2 \pi \sigma_{i}^{2}\right)^{-1 / 2} \exp \left(-\frac{1}{2} \frac{\left(y_{i}-\boldsymbol{x}_{i}^{\prime} \boldsymbol{\beta}\right)^{2}}{\sigma_{i}^{2}}\right)\right]\right\} \\
& =-\frac{1}{2} \sum_{i=1}^{n}\left\{\log (2 \pi)+\log \left(\sigma_{i}^{2}\right)+\left(y_{i}-\boldsymbol{x}_{i}^{\prime} \boldsymbol{\beta}\right)^{2} / \sigma_{i}^{2}\right\}
\end{aligned}
$$




$$
=-\frac{1}{2} \sum_{i=1}^{n}\left\{\log (2 \pi)+\boldsymbol{z}_{i}^{\prime} \boldsymbol{\gamma}+\left(y_{i}-\boldsymbol{x}_{i}^{\prime} \boldsymbol{\beta}\right)^{2} / \exp \left(\boldsymbol{z}_{i}^{\prime} \gamma\right)\right\} .
$$

The authors present a scoring algorithm that can be used to jointly estimate the model parameters. The score vector with respect to $\gamma$ is

$$
S(\boldsymbol{\gamma})=\frac{\partial \ell(\boldsymbol{\gamma})}{\partial \boldsymbol{\gamma}}=\frac{1}{2} \sum_{i=1}^{n} \boldsymbol{z}_{i}\left[\frac{\left(y_{i}-\boldsymbol{x}_{i}^{\prime} \boldsymbol{\beta}\right)^{2}}{\exp \left(\boldsymbol{z}_{i}^{\prime} \boldsymbol{\gamma}\right)}-1\right]
$$

The observed information for $\gamma$ is

$$
K(\gamma)=-\frac{\partial^{2} \ell(\gamma)}{\partial \gamma \partial \gamma^{\prime}}=\frac{1}{2} \sum_{i=1}^{n} z_{i} z_{i}^{\prime}\left[\frac{\left(y_{i}-\boldsymbol{x}_{i}^{\prime} \boldsymbol{\beta}\right)^{2}}{\exp \left(\boldsymbol{z}_{i}^{\prime} \gamma\right)}\right]
$$

The expected information is thus

$$
I(\gamma)=\mathbb{E}[K(\gamma)]=\frac{1}{2} \sum_{i=1}^{n} z_{i} z_{i}^{\prime}
$$

Parameter estimation can now be performed using an iterative scoring algorithm. The estimate of $\gamma$ in the $(k+1)$ th iteration is given by

$$
\gamma_{k+1}=\gamma_{k}+\delta I\left(\gamma_{k}\right)^{-1} S\left(\gamma_{k}\right)=\gamma_{k}+\delta\left(Z^{\prime} Z\right)^{-1} \sum_{i=1}^{n} z_{i}\left[\frac{\left(y_{i}-\boldsymbol{x}_{i}^{\prime} \boldsymbol{\beta}_{k}\right)^{2}}{\exp \left(\boldsymbol{z}_{i}^{\prime} \boldsymbol{\gamma}_{k}\right)}-1\right],
$$

where $0<\delta<1$ is the step length. Given $\gamma_{k+1}$, the parameter vector $\boldsymbol{\beta}$ is re-estimated by weighted least squares. The algorithm stops when convergence is reached.

We shall also consider two alternative estimation approaches, as outlined by Harvey (1976), namely: (i) a two-step procedure and (ii) maximum likelihood estimation. Notice that, the variance function in (3.1) implies that

$$
\Phi=\left[\begin{array}{ccccc}
\exp \left(z_{1}^{\prime} \gamma\right) & 0 & 0 & \ldots & 0 \\
0 & \exp \left(z_{2}^{\prime} \gamma\right) & 0 & \ldots & 0 \\
\vdots & \vdots & \vdots & \ddots & \vdots \\
0 & 0 & 0 & \ldots & \exp \left(z_{n}^{\prime} \gamma\right)
\end{array}\right]
$$

In the two-step procedure, we estimate $\gamma$ from

$$
\log \left(\hat{\epsilon}_{i}^{2}\right)=z_{i}^{\prime} \gamma+u_{i}
$$

where $u_{i}=\log \left(\hat{\epsilon}_{i}^{2} / \sigma_{i}^{2}\right)$. Let $\boldsymbol{q}=\left(\log \left(\hat{\epsilon}_{1}^{2}\right), \ldots, \log \left(\hat{\epsilon}_{n}^{2}\right)\right)^{\prime}$. The estimator of $\gamma$ is

$$
\hat{\gamma}=\left(Z^{\prime} Z\right)^{-1} Z^{\prime} \boldsymbol{q}
$$

Using $\hat{\gamma}$, we obtain an estimator for $\boldsymbol{\beta}$ as follows:

$$
\hat{\hat{\boldsymbol{\beta}}}=\left(X^{\prime} \hat{\Phi}^{-1} X\right)^{-1} X^{\prime} \hat{\Phi}^{-1} \boldsymbol{y}
$$


where $\hat{\Phi}=\operatorname{diag}\left\{\exp \left(\boldsymbol{z}_{1}^{\prime} \hat{\gamma}\right), \ldots, \exp \left(\boldsymbol{z}_{n}^{\prime} \hat{\gamma}\right)\right\}$.

Harvey (1976) showed that the first component of $\hat{\gamma}, \hat{\gamma}_{1}$, is an inconsistent estimator of $\gamma_{1}$, the first component of $\gamma$; in particular, $\operatorname{plim}\left(\hat{\gamma}_{1}\right)=\gamma_{1}-1.2704$. The remaining $s-1$ elements of $\hat{\gamma}$ are, nonetheless, consistent for the corresponding parameters in $\gamma$. Since $\gamma_{1}$ merely introduces a proportionality factor in (3.1), $\hat{\hat{\boldsymbol{\beta}}}$ is consistent for $\boldsymbol{\beta}$.

The third and final approach uses maximum likelihood estimation. The log-likelihood function

$$
\ell(\boldsymbol{\beta}, \boldsymbol{\gamma})=-\frac{1}{2} \sum_{i=1}^{n}\left\{\log (2 \pi)+\boldsymbol{z}_{i}^{\prime} \boldsymbol{\gamma}+\left(y_{i}-\boldsymbol{x}_{i}^{\prime} \boldsymbol{\beta}\right)^{2} / \exp \left(\boldsymbol{z}_{i}^{\prime} \boldsymbol{\gamma}\right)\right\}
$$

is maximized with respect to $\boldsymbol{\beta}$ and $\boldsymbol{\gamma}$. The estimators asymptotic variance-covariance matrix is given by the inverse of Fisher's information matrix:

$$
\left[\begin{array}{cc}
\left(\sum_{i=1}^{n} \sigma_{i}^{-2} \boldsymbol{x}_{i} \boldsymbol{x}_{i}^{\prime}\right)^{-1} & 0 \\
\cdots \cdots \ldots \ldots \ldots \ldots \ldots \ldots \ldots \ldots \ldots \ldots & \ldots \ldots \ldots \\
0 & 2\left(\sum_{i=1}^{n} \boldsymbol{z}_{i} \boldsymbol{z}_{i}^{\prime}\right)^{-1}
\end{array}\right]
$$

In the tables that follow the acronyms EGLS $1, E_{1}$ ELS $_{2}$ and $\mathrm{EGLS}_{3}$ refer to, respectively, maximum likelihood estimation, the estimation procedure outlined by Atkinson, Riani, and Torti (2016) and the two-step estimation procedure. 


\section{Numerical evaluation}

\subsection{Simulation setup}

In what follows we shall numerically evaluate the finite sample performances of quasi- $t$ and $z$ testing inferences in linear regressions. Recall that $z$ test statistics use estimated generalized least squares standard errors whereas quasi- $t$ test statistics use heteroskedasticity-robust standard errors. All reported results are based on 10,000 Monte Carlo simulations and were obtained using the Ox matrix programming language (Doornik 2009). The sample sizes are $n=50,100,150,200$. The covariate values for $n=50$ were replicated twice, three times and four times when $n=100,150,200$, respectively. This was done so that the strength of heteroskedasticity, which is measured by $\lambda=\max \left(\sigma_{i}^{2}\right) / \min \left(\sigma_{i}^{2}\right)$, remains constant as the sample size increases. The values of the dispersion parameters (i.e., the components of $\gamma$ ) in the three experiments were selected so that $\lambda \approx 51$ and $101, \lambda \approx 6$ and 26 and $\lambda=1$ and $\approx 3$, respectively. The simulations thus cover different heteroskedasticity strengths. Following Atkinson, Riani, and Torti (2016), regardless of the true skedastic function, estimated generalized least squares estimation is carried out using

$$
\sigma_{i}^{2}=\exp \left(\sum_{j=1}^{p} \gamma_{j} x_{i j}\right)
$$

In the models used in our simulations, $x_{i 1}=1 \forall i$.

The interest lies in testing the null hypothesis $\mathcal{H}_{0}: \beta_{j}=\beta_{j}^{(0)}$ against the alternative hypothesis $\mathcal{H}_{1}: \beta_{j} \neq \beta_{j}^{(0)}$, for some $j$ in $\{1, \ldots, p\}$, where $\beta_{j}^{(0)}$ is a given scalar. The quasi- $t$ test statistic is

$$
\tau=\frac{\hat{\beta}_{j}-\beta_{j}^{(0)}}{\sqrt{\widehat{\operatorname{var}}\left(\hat{\beta}_{j}\right)}},
$$

where $\widehat{\operatorname{var}}\left(\hat{\beta}_{j}\right)$ denotes the estimated variance of $\hat{\beta}_{j}$ obtained from a heteroskedasticity-consistent covariance matrix estimator. Under the null hypothesis, $\tau$ is asymptotically distributed as $\mathcal{N}(0,1)$. The null hypothesis is rejected if $|\tau|>z_{1-\alpha / 2}$, where $\alpha$ is the test significance level and $z_{1-\alpha / 2}$ denotes the $1-\alpha / 2$ standard normal upper quantile. We consider quasi- $t$ tests based on statistics that use standard errors obtained from $\mathrm{HC} 0, \mathrm{HC} 2, \mathrm{HC} 3, \mathrm{HC} 4$ and $\mathrm{HC} 4 \mathrm{~m}$. We also 
consider testing inferences based on the following test statistic:

$$
\tau_{g}=\frac{\tilde{\beta}_{j g}-\beta_{j}^{(0)}}{\sqrt{\widehat{\operatorname{var}}\left(\tilde{\beta}_{j g}\right)}},
$$

where $\widehat{\operatorname{var}}\left(\tilde{\beta}_{j g}\right)$ is obtained from the estimated covariance matrix of $\tilde{\boldsymbol{\beta}}_{g}, g=1,2,3$ for the three different estimation approaches discussed in Chapter 3. The rejection rule is the same as in the quasi- $t$ tests, i.e., the null hypothesis is rejected if $\left|\tau_{g}\right|>z_{1-\alpha / 2}$. All tests are performed at the $10 \%$ and $5 \%$ significance levels.

The first numerical evaluation uses the following data generating process:

$$
y_{i}=\beta_{1}+\beta_{2} x_{i 2}+\beta_{3} x_{i 3}+\beta_{4} x_{i 4}+\sigma_{i} \epsilon_{i}, \quad i=1, \ldots, n .
$$

All covariate values are absolute values of $\mathcal{N}(0,1)$ random draws. The same covariates are used in the skedastic function. The errors $\epsilon_{i}$ 's are generated from $\mathcal{N}(0,1)$ and from $\chi_{(m)}^{2}, m=2,5,10$; the latter were normalized to have zero mean and unit variance. Data generation is carried out using $\beta_{1}=\beta_{2}=\beta_{3}=\beta_{4}=3$. There is no leverage point. The error variances are

$$
\sigma_{i}^{2}=\exp \left(\gamma+\gamma x_{i 2}+\gamma x_{i 3}+\gamma x_{i 4}\right)
$$

Notice that the skedastic function is equal from that used when performing estimated generalized least squares estimation. We used the following values for $\gamma: 0.935(\lambda \approx 51)$ and $1.101(\lambda \approx$ 101).

The second set of Monte Carlo simulations were based on the model

$$
y_{i}=\beta_{1}+\beta_{2} x_{i}+\sigma_{i} \epsilon_{i}, \quad i=1, \ldots, n .
$$

The covariate values are selected as equally spaced points in $[0,1]$. The largest covariate value (1.0) is then replaced by $a=1.0,2.5,3.5,5.0$. When $a=1.0$, there is no leverage point. When $a>1$, the data are leveraged. The errors $\epsilon_{i}$ 's are randomly generated from the standard normal distribution. Data generation is carried out using $\beta_{1}=\beta_{2}=3$. The skedastic function is

$$
\sigma_{i}^{2}=\exp \left(\gamma+\gamma z_{i}\right)
$$

The values of $z_{1}, \ldots, z_{n}$ are selected as squared $t_{5}$ random draws. Notice that the skedastic function used for performing estimated generalized least squares estimation differs from the true skedastic function. We use the following values of $\gamma: 0.200(\lambda \approx 6)$ and $0.393(\lambda \approx 26)$.

The third Monte Carlo experiment uses

$$
y_{i}=\beta_{1}+\beta_{2} x_{i}+\beta_{3} x_{i}^{2}+\sigma_{i} \epsilon_{i}, \quad i=1, \ldots, n .
$$

The values of $x_{i}$ are those in the empirical application presented in Section 5.2. The data contain three leverage points $(n=50)$. The errors $\epsilon_{i}$ 's are obtained as standard normal random draws. Data generation is performed using $\beta_{1}=-150.868, \beta_{2}=688.806$ and $\beta_{3}=0$. We carried out simulations under homoskedasticity $(\lambda=1)$ and heteroskedasticity $(\lambda>1)$. The skedastic function is

$$
\sigma_{i}^{2}=3700
$$


when the error variances are equal and

$$
\sigma_{i}^{2}=4 \eta_{i}
$$

where $\eta_{i}=\beta_{1}+\beta_{2} x_{i}+\beta_{3} x_{i}^{2}$, under unequal error variances.

\subsection{Size simulations}

Tables 4.1 through 4.5 present the tests null rejection rates (entries are percentages). We consider quasi- $t$ tests (Chapter 2$)$ and also three estimated generalized least squares-based tests (Chapter 3$)$. Consider, at the outset, Tables $4.1(\lambda \approx 51)$ and $4.2(\lambda \approx 101)$ which contain results from the first simulation experiment. We test $\mathcal{H}_{0}: \beta_{3}=3$ against $\mathcal{H}_{1}: \beta_{3} \neq 3$, and present results for normal and nonnormal (chi-squared distributed) errors. The results in Tables 4.1 and 4.2 lead to important conclusions. First, overall, the EGLS tests are the worst performing tests, especially when the sample size is not large $(n=50$ and $n=100)$. Note that the null rejection rates of the three estimated generalized least squares-based tests are very similar. For instance, when $n=50$ and under normal errors, such rejection rates at the $10 \%$ significance level are close to $19 \%$ (in both tables). Under highly asymmetrical errors ( $\chi_{2}^{2}$ distributed errors) the EGLS tests are even more liberal, their null rejection rates becoming approximately equal to $24 \%$ for $E_{\text {EGS }}$ and $21 \%$ for EGLS 2 and EGLS 3 . Second, the performances of the HC0, HC2, HC3, $\mathrm{HC} 4$ and $\mathrm{HC} 4 \mathrm{~m}$ quasi- $t$ tests show some deterioration as the error distribution becomes more asymmetric; such a deterioration is not, however, as pronounced as that of the EGLS tests. For instance, in Table 4.1, when $n=50$ and at the 5\% significance level, the HC0 (HC2) [HC4] null rejection rates for normal and $\chi_{2}^{2}$ errors are $10.67 \%$ and $12.22 \%$ (8.33\% and $10.18 \%$ ) [6.38\% and $8.34 \%$, respectively. Third, overall, all tests become more accurate as the sample size increases.

We now move to the second simulation experiment. The interest lies in testing $\mathcal{H}_{0}: \beta_{2}=3$ against $\mathcal{H}_{1}: \beta_{2} \neq 3$. As before, all tests are carried out at the $10 \%$ and $5 \%$ significance levels. Recall that the regression model contains a single covariate, that for the smallest sample $(n=50)$ its values are selected as an equally spaced sequence of points from 0 to 1 (i.e., in $[0,1]$ ), and that the largest covariate value (1.0) is then replaced by $a=1.0,2.5,3.5,5.0$. We thus aim to evaluate the impact of increased maximal data leverage on the tests finite sample performances. The tests null rejection rates can be found in Tables $4.3(\lambda \approx 6)$ and $4.4(\lambda \approx 26)$. The results we report lead to interesting conclusions. First, the HC0 test becomes much more liberal as the value of $a$ increases, i.e., under progressively more intense maximal leverage, especially when the sample size is not large $(n=50$ and $n=100)$. For instance, when $n=50$ and at the $10 \%$ nominal level, we see in Table 4.3 that the HC0 null rejection rate goes from $12.12 \%$ to $31.02 \%$ when the maximal covariate value goes from 1.0 to 5.0. Second, the EGLS ${ }_{1}$ and EGLS $_{2}$ tests also become progressively more liberal in small samples as the data become more leveraged. Their null rejection rate $(n=50, \alpha=0.10$, Table 4.4) jump from approximately $18 \%$ to approximately $23 \%$ when the value of $a$ is increased from 1.0 to 5.0. Even with larger samples the tests size distortions are substantial. For instance, when $n=200$ and $a=2.5$, the tests null rejection rates are approximately equal to $15 \%$. Third, the $\mathrm{HC} 3, \mathrm{HC} 4$ and $\mathrm{HC} 4 \mathrm{~m}$ tests tend to become more conservative under leveraged data, especially when the sample size 
Table 4.1 Null rejection rates $(\%)$, first simulation experiment; $\lambda \approx 51$.

\begin{tabular}{|c|c|c|c|c|c|c|c|c|c|}
\hline & $n$ & \multicolumn{2}{|c|}{50} & \multicolumn{2}{|c|}{100} & \multicolumn{2}{|c|}{150} & \multicolumn{2}{|c|}{200} \\
\hline Errors & & $5 \%$ & $10 \%$ & $5 \%$ & $10 \%$ & $5 \%$ & $10 \%$ & $5 \%$ & $10 \%$ \\
\hline \multirow{8}{*}{$\mathcal{N}(0,1)$} & $\mathrm{EGLS}_{1}$ & 12.34 & 19.09 & 7.82 & 13.73 & 6.92 & 12.56 & 6.58 & 11.89 \\
\hline & $\mathrm{EGLS}_{2}$ & 12.19 & 18.85 & 7.59 & 13.35 & 6.82 & 12.37 & 6.52 & 11.78 \\
\hline & $\mathrm{EGLS}_{3}$ & 12.03 & 18.38 & 8.29 & 14.11 & 7.32 & 12.82 & 6.73 & 12.05 \\
\hline & $\mathrm{HCO}$ & 10.67 & 16.99 & 8.09 & 14.03 & 7.15 & 12.75 & 6.70 & 12.33 \\
\hline & $\mathrm{HC} 2$ & 8.33 & 13.99 & 7.19 & 12.53 & 6.53 & 11.85 & 6.20 & 11.47 \\
\hline & $\mathrm{HC} 3$ & 6.24 & 11.44 & 6.11 & 11.21 & 5.97 & 11.14 & 5.67 & 10.73 \\
\hline & $\mathrm{HC} 4$ & 6.38 & 11.47 & 6.09 & 11.10 & 5.96 & 11.08 & 5.67 & 10.67 \\
\hline & $\mathrm{HC} 4 \mathrm{~m}$ & 5.66 & 10.50 & 5.65 & 10.68 & 5.68 & 10.75 & 5.55 & 10.41 \\
\hline \multirow{8}{*}{$\chi_{(10)}^{2}$} & $\mathrm{EGLS}_{1}$ & 13.32 & 19.99 & 8.21 & 14.62 & 7.52 & 13.12 & 7.20 & 12.83 \\
\hline & $\mathrm{EGLS}_{2}$ & 12.90 & 19.37 & 7.76 & 13.87 & 7.28 & 12.69 & 6.96 & 12.48 \\
\hline & $\mathrm{EGLS}_{3}$ & 12.60 & 18.85 & 8.89 & 14.25 & 8.03 & 13.52 & 7.61 & 13.11 \\
\hline & $\mathrm{HC} 0$ & 10.98 & 17.07 & 8.47 & 14.01 & 7.26 & 13.10 & 6.84 & 11.96 \\
\hline & $\mathrm{HC} 2$ & 8.75 & 14.24 & 7.57 & 12.68 & 6.74 & 12.18 & 6.46 & 11.25 \\
\hline & $\mathrm{HC} 3$ & 6.82 & 11.70 & 6.58 & 11.33 & 6.19 & 11.30 & 5.98 & 10.67 \\
\hline & $\mathrm{HC} 4$ & 6.90 & 11.77 & 6.58 & 11.32 & 6.16 & 11.28 & 6.00 & 10.61 \\
\hline & $\mathrm{HC} 4 \mathrm{~m}$ & 6.21 & 10.79 & 6.28 & 10.95 & 5.92 & 10.95 & 5.82 & 10.38 \\
\hline \multirow{8}{*}{$\chi_{(5)}^{2}$} & $\mathrm{EGLS}_{1}$ & 14.18 & 20.87 & 9.85 & 15.88 & 8.01 & 13.89 & 6.88 & 12.43 \\
\hline & $\mathrm{EGLS}_{2}$ & 13.63 & 20.00 & 9.09 & 14.65 & 7.49 & 13.07 & 6.63 & 11.98 \\
\hline & $\mathrm{EGLS}_{3}$ & 12.88 & 19.06 & 10.14 & 16.19 & 8.45 & 14.55 & 7.64 & 13.23 \\
\hline & $\mathrm{HC} 0$ & 11.20 & 17.50 & 8.36 & 14.16 & 7.65 & 13.23 & 7.02 & 12.42 \\
\hline & $\mathrm{HC} 2$ & 9.02 & 14.72 & 7.43 & 12.94 & 7.02 & 12.33 & 6.62 & 11.71 \\
\hline & $\mathrm{HC} 3$ & 7.26 & 12.15 & 6.61 & 11.64 & 6.48 & 11.50 & 6.13 & 11.00 \\
\hline & $\mathrm{HC} 4$ & 7.20 & 12.32 & 6.70 & 11.66 & 6.40 & 11.41 & 6.13 & 10.96 \\
\hline & $\mathrm{HC} 4 \mathrm{~m}$ & 6.58 & 11.28 & 6.35 & 11.21 & 6.19 & 11.02 & 5.88 & 10.73 \\
\hline \multirow{8}{*}{$\chi_{(2)}^{2}$} & $\mathrm{EGLS}_{1}$ & 17.31 & 23.54 & 11.05 & 17.28 & 9.59 & 14.97 & 8.28 & 14.10 \\
\hline & $\mathrm{EGLS}_{2}$ & 15.93 & 21.81 & 9.72 & 15.29 & 8.71 & 13.58 & 7.57 & 13.03 \\
\hline & $\mathrm{EGLS}_{3}$ & 14.52 & 21.02 & 11.70 & 17.61 & 10.60 & 16.16 & 9.53 & 15.23 \\
\hline & $\mathrm{HC} 0$ & 12.22 & 18.47 & 9.37 & 15.04 & 8.11 & 13.86 & 7.42 & 12.21 \\
\hline & $\mathrm{HC} 2$ & 10.18 & 15.62 & 8.46 & 13.84 & 7.45 & 12.88 & 6.90 & 11.61 \\
\hline & $\mathrm{HC} 3$ & 8.16 & 13.19 & 7.52 & 12.57 & 6.75 & 12.04 & 6.53 & 11.05 \\
\hline & $\mathrm{HC} 4$ & 8.34 & 13.37 & 7.61 & 12.54 & 6.82 & 11.95 & 6.55 & 11.03 \\
\hline & $\mathrm{HC} 4 \mathrm{~m}$ & 7.45 & 12.37 & 7.21 & 12.15 & 6.49 & 11.71 & 6.41 & 10.80 \\
\hline
\end{tabular}


Table 4.2 Null rejection rates (\%), first simulation experiment; $\lambda \approx 101$.

\begin{tabular}{|c|c|c|c|c|c|c|c|c|c|}
\hline & $n$ & \multicolumn{2}{|c|}{50} & \multicolumn{2}{|c|}{100} & \multicolumn{2}{|c|}{150} & \multicolumn{2}{|c|}{200} \\
\hline Errors & & $5 \%$ & $10 \%$ & $5 \%$ & $10 \%$ & $5 \%$ & $10 \%$ & $5 \%$ & $10 \%$ \\
\hline \multirow{8}{*}{$\mathcal{N}(0,1)$} & $\mathrm{EGLS}_{1}$ & 12.17 & 19.22 & 7.82 & 13.66 & 6.91 & 12.61 & 6.50 & 11.72 \\
\hline & $\mathrm{EGLS}_{2}$ & 11.81 & 18.64 & 7.27 & 12.66 & 6.65 & 12.04 & 6.37 & 11.42 \\
\hline & $\mathrm{EGLS}_{3}$ & 11.61 & 17.99 & 8.33 & 13.68 & 7.02 & 12.59 & 6.61 & 11.84 \\
\hline & $\mathrm{HCO}$ & 10.80 & 17.13 & 8.09 & 14.07 & 7.30 & 12.90 & 6.71 & 12.20 \\
\hline & $\mathrm{HC} 2$ & 8.42 & 14.07 & 7.11 & 12.59 & 6.61 & 12.18 & 6.20 & 11.50 \\
\hline & HC3 & 6.34 & 11.43 & 6.20 & 11.23 & 6.00 & 11.32 & 5.70 & 10.76 \\
\hline & $\mathrm{HC} 4$ & 6.32 & 11.26 & 6.13 & 11.20 & 6.00 & 11.23 & 5.70 & 10.67 \\
\hline & $\mathrm{HC} 4 \mathrm{~m}$ & 5.72 & 10.41 & 5.77 & 10.60 & 5.84 & 10.86 & 5.53 & 10.45 \\
\hline \multirow{8}{*}{$\chi_{(10)}^{2}$} & EGLS $_{1}$ & 13.35 & 19.80 & 8.24 & 14.51 & 7.45 & 13.02 & 7.22 & 12.92 \\
\hline & $\mathrm{EGLS}_{2}$ & 12.62 & 18.73 & 7.38 & 13.01 & 6.96 & 12.18 & 6.70 & 12.08 \\
\hline & $\mathrm{EGLS}_{3}$ & 12.35 & 18.32 & 8.73 & 14.20 & 7.80 & 13.46 & 7.38 & 12.99 \\
\hline & $\mathrm{HCO}$ & 11.12 & 17.46 & 8.67 & 14.33 & 7.46 & 12.94 & 6.86 & 12.01 \\
\hline & $\mathrm{HC} 2$ & 8.81 & 14.46 & 7.59 & 12.74 & 6.78 & 12.08 & 6.35 & 11.34 \\
\hline & $\mathrm{HC} 3$ & 6.85 & 11.80 & 6.75 & 11.38 & 6.28 & 11.25 & 5.88 & 10.68 \\
\hline & $\mathrm{HC} 4$ & 6.88 & 11.85 & 6.63 & 11.34 & 6.22 & 11.17 & 5.87 & 10.60 \\
\hline & $\mathrm{HC} 4 \mathrm{~m}$ & 6.21 & 10.80 & 6.31 & 10.93 & 6.09 & 10.89 & 5.78 & 10.39 \\
\hline \multirow{8}{*}{$\chi_{(5)}^{2}$} & $\mathrm{EGLS}_{1}$ & 14.30 & 20.97 & 9.93 & 15.78 & 7.88 & 13.80 & 6.95 & 12.18 \\
\hline & $\mathrm{EGLS}_{2}$ & 13.26 & 19.47 & 8.73 & 13.84 & 7.11 & 12.48 & 6.49 & 11.41 \\
\hline & $\mathrm{EGLS}_{3}$ & 12.76 & 18.83 & 10.02 & 15.89 & 8.36 & 14.61 & 7.50 & 13.01 \\
\hline & $\mathrm{HCO}$ & 11.53 & 17.95 & 8.47 & 14.40 & 7.71 & 13.36 & 7.19 & 12.56 \\
\hline & $\mathrm{HC} 2$ & 9.37 & 15.08 & 7.57 & 13.07 & 7.15 & 12.43 & 6.78 & 11.77 \\
\hline & $\mathrm{HC} 3$ & 7.31 & 12.34 & 6.66 & 11.76 & 6.57 & 11.51 & 6.30 & 11.08 \\
\hline & $\mathrm{HC} 4$ & 7.31 & 12.31 & 6.68 & 11.69 & 6.51 & 11.40 & 6.21 & 11.02 \\
\hline & $\mathrm{HC} 4 \mathrm{~m}$ & 6.68 & 11.45 & 6.44 & 11.26 & 6.27 & 11.06 & 5.99 & 10.75 \\
\hline \multirow{8}{*}{$\chi_{(2)}^{2}$} & $\mathrm{EGLS}_{1}$ & 17.04 & 23.40 & 11.06 & 17.34 & 9.61 & 14.93 & 8.42 & 14.10 \\
\hline & $\mathrm{EGLS}_{2}$ & 15.09 & 20.79 & 9.05 & 14.36 & 8.21 & 12.80 & 7.25 & 12.33 \\
\hline & $\mathrm{EGLS}_{3}$ & 14.69 & 20.99 & 11.51 & 17.37 & 10.32 & 16.27 & 9.81 & 15.51 \\
\hline & $\mathrm{HCO}$ & 12.64 & 19.13 & 9.76 & 15.22 & 8.41 & 14.04 & 7.60 & 12.57 \\
\hline & $\mathrm{HC} 2$ & 10.66 & 16.11 & 8.74 & 14.16 & 7.78 & 13.17 & 7.21 & 12.00 \\
\hline & HC3 & 8.67 & 13.59 & 7.87 & 13.00 & 7.12 & 12.28 & 6.79 & 11.38 \\
\hline & $\mathrm{HC} 4$ & 8.79 & 13.60 & 7.88 & 12.92 & 7.13 & 12.30 & 6.78 & 11.37 \\
\hline & $\mathrm{HC} 4 \mathrm{~m}$ & 7.79 & 12.69 & 7.49 & 12.52 & 6.88 & 11.90 & 6.67 & 11.15 \\
\hline
\end{tabular}


is not large. To illustrate that, consider $n=50$ and $\alpha=0.10$ in Table 4.4. For $a=1.0$, the three tests null rejection rates are $9.81 \%, 10.34 \%$ and $9.43 \%$, respectively; when $a=5.0$, the corresponding figures are $3.16 \%, 0.47 \%$ and $2.05 \%$. The HC4 is particularly affected by the extreme leverage. Fourth, the tests size distortions tend to decrease as $n$ increases.

The tests null rejection rates obtained in the third simulation scenario are presented in Table 4.5 (entries are percentages). The interest lies in testing $\mathcal{H}_{0}: \beta_{3}=0$ against $\mathcal{H}_{1}: \beta_{3} \neq 0$. Recall that we are testing a linear versus a quadratic functional form. We present results obtained under homoskedasticity $\left(\lambda=1.0, \sigma^{2}=3700\right)$ and mild heteroskedasticity $(\lambda \approx 2.5$, $\left.\sigma_{i}^{2}=4 \eta_{i}\right)$. The first conclusion we draw from the figures in Table 4.5 is that the EGLS tests are again considerably liberal (especially EGLS ${ }_{1}$ and EGLS $S_{2}$ in small samples), even when the sample size is large $(n=200)$. For instance, under homoskedasticity (heteroskedasticity) and at the $10 \%$ significance level the null rejection rates of the of the EGLS ${ }_{1}$ test when for $n=50$ and $n=200$ are, respectively, $36.53 \%$ and $14.42 \%$ (37.78\% and 14.89\%). Second, the HC0 and HC2 tests are again liberal, even when $n=200$. Third, the HC4 test is once again conservative. Fourth, overall, the HC3 and $\mathrm{HC} 4 \mathrm{~m}$ tests display good control of the type I error frequency. For example, under heteroskedasticity and when $n=100$, the HC3 and HC4m null rejection rates at the $10 \%$ significance level are, respectively, $11.15 \%$ and $9.91 \%$.

\subsection{Power simulations}

We shall now focus on the tests power, i.e., on the tests ability to detect that the null hypothesis is false when it is indeed false. We performed Monte Carlo simulations using the same scenarios as in the size simulations. Data generation, however, is now carried out under the alternative hypothesis. Since some tests are considerably liberal, we compare the powers of size-corrected tests. At the outset, we consider the setup used in the first set of size simulations. We test $\mathcal{H}_{0}$ : $\beta_{3}=3$ but the true parameter value is taken to be 6.0, i.e., data generation is performed using $\beta_{3}=6.0$. Tables 4.6 and 4.7 contain the tests nonnull rejection rates (entries are percentages). The EGLS tests are more powerful than the quasi- $t$ tests regardless of the error distribution and sample size. We also note that the powers of all test increase with the sample size, as expected.

Simulation results for the second scenario are presented in Tables 4.8 and 4.9 (entries are percentages). Recall that the maximal leverage increases with $a$. We test $\mathcal{H}_{0}: \beta_{2}=3$ against a two-sided alternative hypothesis, and data generation is carried out using $\beta_{2}=4.1$. The numerical results reported in the two tables show that the quasi- $t$ tests are slightly less powerful than the EGLS $S_{1}$ and EGLS 2 tests when $a=1.0$, i.e., when the data do not contain a leverage point. For instance, when $n=150$ and at the $10 \%$ significance level the powers of the EGLS and EGLS $S_{2}$ tests are approximately $39 \%$ whereas the powers of the quasi- $t$ tests are around $37 \%$ (Table 4.9). As the data become more leveraged (i.e., as the value of $a$ increases), however, some quasi- $t$ tests become progressively more powerful than the EGLS tests. Take, for instance, $n=100, a=5.0$ and $\alpha=0.10$ (Table 4.9). The EGLS $1, \mathrm{EGLS}_{2}$ and EGLS 3 tests nonnull rejection rates are $45.08 \%, 45.14 \%$ and $80.77 \%$, respectively; the corresponding figure for the $\mathrm{HCO}(\mathrm{HC} 3)$ [HC4m] test is $99.30 \%(97.50 \%)$ [96.50\%]. It is also noteworthy that the HC4 test is typically the least powerful quasi- $t$ test when the sample size is small $(n=50)$. Additionally, in small samples and under strongly leveraged data, the EGLS 3 test is more powerful than the $E^{E G S} S_{1}$ and EGLS $S_{2}$ tests. When $n \geq 100$, all quasi- $t$ tests are nearly equally powerful. 
Table 4.3 Null rejection rates (\%), second simulation experiment; $\lambda \approx 6$.

\begin{tabular}{|c|c|c|c|c|c|c|c|c|c|}
\hline & $n$ & \multicolumn{2}{|c|}{50} & \multicolumn{2}{|c|}{100} & \multicolumn{2}{|c|}{150} & \multicolumn{2}{|c|}{200} \\
\hline$a$ & & $5 \%$ & $10 \%$ & $5 \%$ & $10 \%$ & $5 \%$ & $10 \%$ & $5 \%$ & $10 \%$ \\
\hline \multirow{8}{*}{1.0} & $\mathrm{EGLS}_{1}$ & 8.42 & 14.42 & 7.40 & 13.46 & 7.68 & 13.60 & 7.28 & 13.22 \\
\hline & $\mathrm{EGLS}_{2}$ & 8.42 & 14.42 & 7.40 & 13.46 & 7.68 & 13.60 & 7.28 & 13.22 \\
\hline & $\mathrm{EGLS}_{3}$ & 9.93 & 16.26 & 8.81 & 15.11 & 9.04 & 15.53 & 8.71 & 15.07 \\
\hline & $\mathrm{HCO}$ & 6.64 & 12.12 & 5.76 & 11.37 & 5.98 & 11.26 & 5.77 & 10.73 \\
\hline & $\mathrm{HC} 2$ & 5.98 & 11.07 & 5.46 & 10.81 & 5.73 & 10.91 & 5.62 & 10.53 \\
\hline & HC3 & 5.45 & 10.17 & 5.11 & 10.19 & 5.52 & 10.60 & 5.42 & 10.27 \\
\hline & $\mathrm{HC} 4$ & 5.70 & 10.58 & 5.30 & 10.47 & 5.59 & 10.74 & 5.52 & 10.41 \\
\hline & $\mathrm{HC} 4 \mathrm{~m}$ & 5.22 & 9.80 & 4.98 & 10.00 & 5.38 & 10.49 & 5.31 & 10.20 \\
\hline \multirow{8}{*}{2.5} & $\mathrm{EGLS}_{1}$ & 11.11 & 16.93 & 6.51 & 11.63 & 5.73 & 11.08 & 5.10 & 10.28 \\
\hline & $\mathrm{EGLS}_{2}$ & 11.10 & 16.92 & 6.51 & 11.63 & 5.73 & 11.08 & 5.10 & 10.28 \\
\hline & $\mathrm{EGLS}_{3}$ & 13.75 & 20.03 & 9.50 & 15.27 & 8.19 & 14.01 & 7.31 & 12.94 \\
\hline & $\mathrm{HC} 0$ & 8.87 & 15.26 & 6.96 & 12.58 & 6.82 & 12.01 & 6.34 & 11.85 \\
\hline & $\mathrm{HC} 2$ & 6.87 & 11.64 & 5.79 & 11.01 & 6.00 & 10.95 & 5.72 & 11.10 \\
\hline & $\mathrm{HC} 3$ & 4.95 & 8.66 & 4.75 & 9.38 & 5.08 & 9.79 & 5.17 & 10.22 \\
\hline & $\mathrm{HC} 4$ & 2.64 & 4.89 & 3.36 & 6.73 & 3.94 & 8.30 & 4.41 & 8.70 \\
\hline & $\mathrm{HC} 4 \mathrm{~m}$ & 4.16 & 7.62 & 4.39 & 8.68 & 4.73 & 9.32 & 4.90 & 9.90 \\
\hline \multirow{8}{*}{3.5} & $\mathrm{EGLS}_{1}$ & 19.21 & 25.15 & 8.36 & 13.44 & 5.95 & 10.48 & 4.65 & 9.54 \\
\hline & $\mathrm{EGLS}_{2}$ & 19.26 & 25.20 & 8.36 & 13.43 & 5.95 & 10.46 & 4.65 & 9.55 \\
\hline & $\mathrm{EGLS}_{3}$ & 17.13 & 24.14 & 11.33 & 16.72 & 8.60 & 14.00 & 7.64 & 13.23 \\
\hline & $\mathrm{HCO}$ & 13.84 & 20.84 & 9.68 & 16.22 & 8.45 & 14.53 & 8.01 & 13.79 \\
\hline & $\mathrm{HC} 2$ & 8.74 & 13.89 & 7.45 & 12.95 & 7.04 & 12.29 & 6.97 & 12.00 \\
\hline & $\mathrm{HC} 3$ & 4.95 & 8.09 & 5.62 & 9.90 & 5.65 & 10.25 & 5.91 & 10.53 \\
\hline & $\mathrm{HC} 4$ & 1.79 & 2.88 & 3.07 & 5.43 & 3.48 & 6.80 & 4.01 & 7.89 \\
\hline & $\mathrm{HC} 4 \mathrm{~m}$ & 3.84 & 6.29 & 4.91 & 8.37 & 5.07 & 9.27 & 5.49 & 9.69 \\
\hline \multirow{8}{*}{5.0} & $\mathrm{EGLS}_{1}$ & 34.82 & 41.11 & 13.47 & 18.87 & 7.94 & 12.30 & 6.00 & 10.09 \\
\hline & $\mathrm{EGLS}_{2}$ & 35.28 & 41.52 & 13.49 & 18.89 & 7.94 & 12.30 & 5.99 & 10.10 \\
\hline & $\mathrm{EGLS}_{3}$ & 22.31 & 28.98 & 13.04 & 17.89 & 9.88 & 14.37 & 8.91 & 13.72 \\
\hline & $\mathrm{HCO}$ & 23.49 & 31.02 & 15.16 & 21.86 & 11.99 & 18.12 & 10.78 & 16.62 \\
\hline & $\mathrm{HC} 2$ & 11.87 & 16.91 & 11.00 & 16.81 & 9.19 & 14.73 & 8.84 & 14.09 \\
\hline & $\mathrm{HC} 3$ & 5.06 & 7.35 & 7.89 & 11.99 & 6.96 & 11.56 & 6.91 & 11.62 \\
\hline & $\mathrm{HC} 4$ & 0.81 & 1.27 & 3.40 & 5.59 & 3.60 & 6.43 & 4.12 & 7.35 \\
\hline & $\mathrm{HC} 4 \mathrm{~m}$ & 3.24 & 4.55 & 6.32 & 10.07 & 6.00 & 10.08 & 6.17 & 10.57 \\
\hline
\end{tabular}


Table 4.4 Null rejection rates (\%), second simulation experiment; $\lambda \approx 26$.

\begin{tabular}{|c|c|c|c|c|c|c|c|c|c|}
\hline & $n$ & \multicolumn{2}{|c|}{50} & \multicolumn{2}{|c|}{100} & \multicolumn{2}{|c|}{150} & \multicolumn{2}{|c|}{200} \\
\hline$a$ & & $5 \%$ & $10 \%$ & $5 \%$ & $10 \%$ & $5 \%$ & $10 \%$ & $5 \%$ & $10 \%$ \\
\hline \multirow{8}{*}{1.0} & $\mathrm{EGLS}_{1}$ & 10.72 & 17.81 & 10.30 & 17.05 & 9.90 & 16.91 & 9.56 & 16.69 \\
\hline & $\mathrm{EGLS}_{2}$ & 10.72 & 17.81 & 10.30 & 17.05 & 9.90 & 16.91 & 9.56 & 16.69 \\
\hline & $\mathrm{EGLS}_{3}$ & 15.94 & 24.01 & 16.43 & 24.37 & 16.91 & 25.02 & 16.36 & 24.51 \\
\hline & $\mathrm{HCO}$ & 6.60 & 11.89 & 5.73 & 11.53 & 5.88 & 11.61 & 5.45 & 11.23 \\
\hline & $\mathrm{HC} 2$ & 5.73 & 10.76 & 5.40 & 11.15 & 5.63 & 11.20 & 5.29 & 11.06 \\
\hline & HC3 & 5.06 & 9.81 & 4.98 & 10.56 & 5.44 & 10.85 & 5.17 & 10.81 \\
\hline & $\mathrm{HC} 4$ & 5.35 & 10.34 & 5.24 & 10.84 & 5.53 & 10.99 & 5.25 & 10.92 \\
\hline & $\mathrm{HC} 4 \mathrm{~m}$ & 4.83 & 9.43 & 4.83 & 10.42 & 5.35 & 10.71 & 5.09 & 10.69 \\
\hline \multirow{8}{*}{2.5} & $\mathrm{EGLS}_{1}$ & 9.97 & 16.58 & 8.78 & 15.15 & 8.63 & 15.38 & 8.48 & 15.03 \\
\hline & $\mathrm{EGLS}_{2}$ & 9.97 & 16.58 & 8.78 & 15.15 & 8.63 & 15.38 & 8.48 & 15.03 \\
\hline & $\mathrm{EGLS}_{3}$ & 16.20 & 24.18 & 14.39 & 22.00 & 13.66 & 21.14 & 13.21 & 20.87 \\
\hline & $\mathrm{HCO}$ & 4.68 & 9.74 & 4.19 & 9.34 & 4.67 & 9.95 & 4.83 & 10.22 \\
\hline & $\mathrm{HC} 2$ & 3.10 & 6.51 & 3.20 & 7.97 & 3.98 & 8.91 & 4.25 & 9.50 \\
\hline & $\mathrm{HC} 3$ & 1.97 & 4.30 & 2.53 & 6.32 & 3.39 & 7.94 & 3.84 & 8.90 \\
\hline & $\mathrm{HC} 4$ & 1.00 & 2.14 & 1.51 & 4.19 & 2.39 & 6.41 & 3.27 & 7.58 \\
\hline & $\mathrm{HC} 4 \mathrm{~m}$ & 1.69 & 3.61 & 2.17 & 5.74 & 3.12 & 7.54 & 3.76 & 8.64 \\
\hline \multirow{8}{*}{3.5} & $\mathrm{EGLS}_{1}$ & 11.78 & 17.88 & 8.74 & 14.74 & 8.41 & 14.92 & 8.17 & 14.32 \\
\hline & $\mathrm{EGLS}_{2}$ & 11.78 & 17.89 & 8.74 & 14.74 & 8.40 & 14.92 & 8.17 & 14.32 \\
\hline & $\mathrm{EGLS}_{3}$ & 17.55 & 25.52 & 13.70 & 20.55 & 11.44 & 18.07 & 10.63 & 17.35 \\
\hline & $\mathrm{HCO}$ & 6.47 & 11.99 & 5.33 & 10.54 & 5.22 & 10.45 & 5.27 & 10.72 \\
\hline & $\mathrm{HC} 2$ & 3.27 & 6.61 & 3.60 & 8.09 & 4.07 & 8.75 & 4.41 & 9.45 \\
\hline & $\mathrm{HC} 3$ & 1.47 & 3.22 & 2.38 & 5.65 & 3.21 & 7.11 & 3.65 & 8.08 \\
\hline & $\mathrm{HC} 4$ & 0.44 & 1.00 & 1.23 & 2.68 & 1.64 & 4.33 & 2.32 & 5.90 \\
\hline & $\mathrm{HC} 4 \mathrm{~m}$ & 1.04 & 2.38 & 2.06 & 4.65 & 2.82 & 6.42 & 3.32 & 7.56 \\
\hline \multirow{8}{*}{5.0} & $\mathrm{EGLS}_{1}$ & 16.91 & 23.11 & 9.22 & 15.09 & 8.27 & 14.01 & 7.74 & 13.16 \\
\hline & $\mathrm{EGLS}_{2}$ & 16.99 & 23.17 & 9.22 & 15.07 & 8.27 & 14.00 & 7.73 & 13.16 \\
\hline & $\mathrm{EGLS}_{3}$ & 18.34 & 25.39 & 12.00 & 17.86 & 8.86 & 14.41 & 7.81 & 12.97 \\
\hline & $\mathrm{HCO}$ & 12.36 & 19.10 & 8.82 & 15.20 & 7.83 & 13.42 & 7.34 & 12.92 \\
\hline & $\mathrm{HC} 2$ & 5.09 & 8.33 & 6.03 & 10.80 & 5.78 & 10.66 & 5.89 & 10.95 \\
\hline & $\mathrm{HC} 3$ & 1.96 & 3.16 & 3.89 & 7.14 & 4.11 & 8.06 & 4.64 & 8.93 \\
\hline & $\mathrm{HC} 4$ & 0.24 & 0.47 & 1.43 & 2.93 & 2.03 & 4.02 & 2.55 & 5.46 \\
\hline & $\mathrm{HC} 4 \mathrm{~m}$ & 1.26 & 2.05 & 3.18 & 5.80 & 3.30 & 7.05 & 3.97 & 8.08 \\
\hline
\end{tabular}


Table 4.5 Null rejection rates (\%), third simulation experiment; homoskedasticity and heteroskedasticity.

\begin{tabular}{|c|c|c|c|c|c|c|c|c|c|}
\hline & $n$ & \multicolumn{2}{|c|}{50} & \multicolumn{2}{c|}{100} & \multicolumn{2}{c|}{150} & \multicolumn{2}{|c|}{200} \\
\hline$\lambda$ & & $5 \%$ & $10 \%$ & $5 \%$ & $10 \%$ & $5 \%$ & $10 \%$ & $5 \%$ & $10 \%$ \\
\hline \multirow{6}{*}{1.00} & EGLS $_{1}$ & 29.36 & 36.53 & 12.58 & 19.21 & 10.00 & 15.95 & 8.40 & 14.42 \\
& EGLS $_{2}$ & 29.33 & 36.55 & 12.58 & 19.21 & 9.99 & 15.95 & 8.40 & 14.42 \\
& EGLS $_{3}$ & 15.11 & 21.49 & 10.24 & 15.91 & 9.18 & 14.48 & 8.33 & 13.25 \\
& HC0 & 13.90 & 20.62 & 9.16 & 15.12 & 8.51 & 14.13 & 7.35 & 12.80 \\
& HC2 & 9.51 & 15.09 & 7.11 & 12.43 & 7.17 & 12.33 & 6.33 & 11.53 \\
& HC3 & 5.79 & 9.46 & 5.34 & 9.83 & 6.01 & 10.79 & 5.56 & 10.14 \\
& HC4 & 1.98 & 3.53 & 2.94 & 5.83 & 4.33 & 7.91 & 4.06 & 8.02 \\
& HC4m & 4.48 & 7.49 & 4.54 & 8.72 & 5.49 & 9.94 & 5.19 & 9.58 \\
\hline \multirow{6}{*}{2.44} & EGLS & 30.90 & 37.78 & 13.32 & 20.33 & 10.64 & 16.28 & 8.76 & 14.89 \\
& EGLS & 30.82 & 37.77 & 13.32 & 20.33 & 10.64 & 16.28 & 8.76 & 14.89 \\
& EGLS & 16.63 & 23.44 & 11.25 & 16.85 & 10.13 & 15.24 & 8.66 & 14.16 \\
& HC0 & 17.55 & 24.63 & 11.00 & 17.63 & 9.80 & 15.59 & 8.37 & 14.35 \\
& HC2 & 11.83 & 18.26 & 8.63 & 14.26 & 8.25 & 13.55 & 7.07 & 12.43 \\
& HC3 & 7.35 & 11.45 & 6.40 & 11.15 & 6.82 & 11.51 & 6.10 & 11.01 \\
& HC4 & 2.38 & 4.15 & 3.48 & 6.55 & 4.70 & 8.11 & 4.19 & 8.17 \\
& HC4m & 5.69 & 9.07 & 5.59 & 9.91 & 6.29 & 10.55 & 5.73 & 10.15 \\
\hline
\end{tabular}

The tests nonnonull rejection rates for the third simulation scenario are presented in Table 4.10 (entries are percentages). We test $\mathcal{H}_{0}: \beta_{3}=0$ against a two-sided alternative hypothesis and the true parameter value is taken to be 1100 . (Recall that the values of the other two regression parameters are $\beta_{1}=-150.868$ and $\beta_{2}=688.806$.) Again, HC4 is the least powerful test when the sample size is small. When $n \geq 100$, all tests are nearly equally powerful.

\subsection{Variability}

In the previous sections we focused on testing inferences. It should be noted, however, that the variances of the ordinary and estimated generalized least squares estimators of the parameters that index the linear regression models can be considerably distinct. The estimators of such variances can also behave quite differently in finite samples. In order to show that, we consider the second simulation experiment used in the size and power simulations and present in Table 4.11 the true variances of the two estimators of $\beta_{2}$ for $a=1.0,5.0$; recall that when $a=5.0$ the data are substantially leveraged. The sample sizes are $n=50,200$. It is noteworthy that the EGLS $_{2}$ estimator of the regression slope parameter is more accurate than the OLS estimator. The difference between the two variances is considerably large when $\lambda \approx 25$ and $a=1.0$ (no leverage point in the data): for $n=50, \operatorname{var}\left(\hat{\beta}_{2}\right)=1.4085$ and $\operatorname{var}\left(\tilde{\beta}_{2}\right)=0.5842$, a difference of almost $140 \%$. Nonetheless, such a difference nearly vanishes as the data become substantially leveraged: when $\lambda \approx 25, n=50$ and $a=5.0, \operatorname{var}\left(\hat{\beta}_{2}\right)=0.0884$ and $\operatorname{var}\left(\tilde{\beta}_{2}\right)=0.0665$. Notice that under strong leverage the true variances are considerably smaller than under well balanced data, i.e., the OLS and EGLS estimators of $\beta_{2}$ fluctuate considerably less. Additionally, the two estimators become approximately equally accurate. 
Table 4.6 Nonnull rejection rates $(\%)$, first simulation experiment; $\lambda \approx 51$.

\begin{tabular}{|c|c|c|c|c|c|c|c|c|c|}
\hline & $n$ & \multicolumn{2}{|c|}{50} & \multicolumn{2}{|c|}{100} & \multicolumn{2}{|c|}{150} & \multicolumn{2}{|c|}{200} \\
\hline Errors & & $5 \%$ & $10 \%$ & $5 \%$ & $10 \%$ & $5 \%$ & $10 \%$ & $5 \%$ & $10 \%$ \\
\hline \multirow{8}{*}{$\mathcal{N}(0,1)$} & EGLS $_{1}$ & 45.89 & 59.65 & 86.77 & 92.33 & 97.40 & 98.79 & 99.58 & 99.80 \\
\hline & $\mathrm{EGLS}_{2}$ & 45.05 & 58.97 & 84.62 & 90.03 & 96.23 & 97.63 & 98.99 & 99.21 \\
\hline & $\mathrm{EGLS}_{3}$ & 34.53 & 50.65 & 80.22 & 88.67 & 95.77 & 97.85 & 99.17 & 99.68 \\
\hline & $\mathrm{HCO}$ & 32.47 & 45.41 & 64.86 & 73.61 & 81.54 & 88.95 & 93.61 & 96.15 \\
\hline & $\mathrm{HC} 2$ & 31.97 & 45.08 & 64.62 & 73.45 & 81.48 & 88.86 & 93.51 & 96.14 \\
\hline & HC3 & 31.34 & 44.62 & 64.41 & 73.31 & 81.30 & 88.84 & 93.47 & 96.13 \\
\hline & $\mathrm{HC} 4$ & 30.92 & 43.90 & 64.08 & 73.08 & 81.19 & 88.82 & 93.44 & 96.09 \\
\hline & $\mathrm{HC} 4 \mathrm{~m}$ & 30.83 & 44.35 & 64.29 & 73.17 & 81.16 & 88.87 & 93.44 & 96.13 \\
\hline \multirow{8}{*}{$\chi_{(10)}^{2}$} & $\mathrm{EGLS}_{1}$ & 38.56 & 53.94 & 86.24 & 92.80 & 97.71 & 98.95 & 99.76 & 99.91 \\
\hline & $\mathrm{EGLS}_{2}$ & 37.55 & 52.54 & 82.25 & 88.60 & 94.83 & 96.01 & 97.89 & 98.02 \\
\hline & $\mathrm{EGLS}_{3}$ & 27.95 & 43.90 & 79.29 & 88.74 & 95.68 & 98.28 & 99.48 & 99.81 \\
\hline & $\mathrm{HC} 0$ & 26.95 & 39.54 & 62.21 & 74.72 & 85.03 & 91.85 & 95.16 & 97.85 \\
\hline & $\mathrm{HC} 2$ & 26.41 & 38.69 & 61.82 & 74.50 & 84.97 & 91.83 & 95.14 & 97.84 \\
\hline & $\mathrm{HC} 3$ & 25.88 & 37.98 & 61.41 & 74.46 & 84.90 & 91.78 & 95.12 & 97.83 \\
\hline & $\mathrm{HC} 4$ & 25.03 & 37.17 & 60.57 & 74.39 & 84.73 & 91.72 & 95.03 & 97.80 \\
\hline & $\mathrm{HC} 4 \mathrm{~m}$ & 25.22 & 37.51 & 60.85 & 74.43 & 84.85 & 91.76 & 95.03 & 97.81 \\
\hline \multirow{8}{*}{$\chi_{(5)}^{2}$} & $\mathrm{EGLS}_{1}$ & 33.37 & 52.28 & 85.73 & 92.54 & 98.22 & 99.38 & 99.81 & 99.95 \\
\hline & $\mathrm{EGLS}_{2}$ & 31.61 & 49.54 & 80.11 & 86.36 & 93.46 & 94.53 & 96.21 & 96.35 \\
\hline & $\mathrm{EGLS}_{3}$ & 26.70 & 45.22 & 79.70 & 89.19 & 96.66 & 98.65 & 99.57 & 99.89 \\
\hline & $\mathrm{HCO}$ & 23.83 & 38.71 & 59.84 & 75.08 & 84.45 & 93.21 & 96.12 & 98.57 \\
\hline & $\mathrm{HC} 2$ & 23.34 & 38.22 & 58.93 & 74.81 & 84.40 & 93.16 & 96.09 & 98.58 \\
\hline & $\mathrm{HC} 3$ & 22.87 & 37.39 & 58.77 & 74.66 & 84.14 & 93.12 & 96.03 & 98.55 \\
\hline & $\mathrm{HC} 4$ & 22.24 & 36.11 & 58.62 & 74.56 & 84.07 & 93.09 & 96.07 & 98.58 \\
\hline & $\mathrm{HC} 4 \mathrm{~m}$ & 22.49 & 36.55 & 58.52 & 74.64 & 84.01 & 93.12 & 96.02 & 98.55 \\
\hline \multirow{8}{*}{$\chi_{(2)}^{2}$} & $\mathrm{EGLS}_{1}$ & 20.00 & 42.93 & 79.62 & 92.18 & 97.98 & 99.48 & 99.80 & 99.95 \\
\hline & $\mathrm{EGLS}_{2}$ & 19.11 & 41.47 & 72.74 & 82.86 & 89.72 & 90.94 & 92.86 & 92.97 \\
\hline & $\mathrm{EGLS}_{3}$ & 19.06 & 38.51 & 74.19 & 88.29 & 96.32 & 98.96 & 99.65 & 99.88 \\
\hline & $\mathrm{HCO}$ & 21.81 & 36.60 & 58.44 & 78.84 & 88.24 & 95.61 & 96.61 & 99.17 \\
\hline & $\mathrm{HC} 2$ & 20.86 & 35.18 & 57.78 & 78.35 & 88.01 & 95.47 & 96.61 & 99.16 \\
\hline & $\mathrm{HC} 3$ & 19.90 & 34.40 & 56.87 & 78.13 & 87.87 & 95.40 & 96.54 & 99.16 \\
\hline & $\mathrm{HC} 4$ & 18.78 & 33.23 & 56.18 & 77.55 & 87.71 & 95.36 & 96.50 & 99.14 \\
\hline & $\mathrm{HC} 4 \mathrm{~m}$ & 19.25 & 33.87 & 56.37 & 77.84 & 87.76 & 95.38 & 96.50 & 99.16 \\
\hline
\end{tabular}


Table 4.7 Nonnull rejection rates (\%), first simulation experiment; $\lambda \approx 101$.

\begin{tabular}{|c|c|c|c|c|c|c|c|c|c|}
\hline & $n$ & \multicolumn{2}{|c|}{50} & \multicolumn{2}{|c|}{100} & \multicolumn{2}{|c|}{150} & \multicolumn{2}{|c|}{200} \\
\hline Errors & & $5 \%$ & $10 \%$ & $5 \%$ & $10 \%$ & $5 \%$ & $10 \%$ & $5 \%$ & $10 \%$ \\
\hline \multirow{8}{*}{$\mathcal{N}(0,1)$} & $\mathrm{EGLS}_{1}$ & 26.81 & 38.69 & 62.38 & 73.43 & 83.08 & 89.51 & 92.79 & 96.12 \\
\hline & $\mathrm{EGLS}_{2}$ & 25.95 & 37.24 & 58.39 & 68.97 & 80.32 & 86.45 & 90.89 & 94.25 \\
\hline & $\mathrm{EGLS}_{3}$ & 18.97 & 30.58 & 54.33 & 66.85 & 77.72 & 86.25 & 90.77 & 94.90 \\
\hline & $\mathrm{HC} 0$ & 16.22 & 25.51 & 34.86 & 45.01 & 48.24 & 60.18 & 64.03 & 73.24 \\
\hline & $\mathrm{HC} 2$ & 16.05 & 25.13 & 34.83 & 45.00 & 48.09 & 60.19 & 64.00 & 73.24 \\
\hline & HC3 & 15.82 & 24.79 & 34.36 & 44.76 & 48.06 & 60.23 & 63.93 & 73.27 \\
\hline & HC4 & 15.65 & 24.71 & 34.24 & 44.53 & 48.03 & 60.07 & 63.89 & 73.23 \\
\hline & $\mathrm{HC} 4 \mathrm{~m}$ & 15.76 & 24.55 & 34.17 & 44.67 & 48.00 & 60.15 & 63.88 & 73.27 \\
\hline \multirow{8}{*}{$\chi_{(10)}^{2}$} & $\mathrm{EGLS}_{1}$ & 19.11 & 31.23 & 56.49 & 71.10 & 81.15 & 88.95 & 93.28 & 96.19 \\
\hline & $\mathrm{EGLS}_{2}$ & 18.63 & 29.91 & 53.17 & 65.95 & 76.44 & 83.74 & 89.53 & 92.17 \\
\hline & $\mathrm{EGLS}_{3}$ & 12.93 & 23.80 & 47.36 & 62.67 & 76.02 & 85.82 & 90.32 & 94.51 \\
\hline & $\mathrm{HC} 0$ & 11.60 & 19.13 & 26.04 & 38.98 & 45.25 & 59.10 & 62.22 & 74.32 \\
\hline & $\mathrm{HC} 2$ & 11.40 & 18.66 & 25.77 & 38.89 & 45.22 & 58.91 & 62.08 & 74.34 \\
\hline & $\mathrm{HC} 3$ & 10.95 & 18.29 & 25.56 & 38.86 & 45.07 & 58.90 & 61.80 & 74.32 \\
\hline & HC4 & 10.63 & 18.02 & 25.10 & 38.78 & 44.81 & 58.80 & 61.64 & 74.22 \\
\hline & $\mathrm{HC} 4 \mathrm{~m}$ & 10.81 & 18.13 & 25.49 & 38.95 & 44.91 & 58.77 & 61.88 & 74.28 \\
\hline \multirow{8}{*}{$\chi_{(5)}^{2}$} & $\mathrm{EGLS}_{1}$ & 14.08 & 28.12 & 55.53 & 68.84 & 82.36 & 89.93 & 93.69 & 97.00 \\
\hline & $\mathrm{EGLS}_{2}$ & 13.07 & 26.36 & 49.20 & 60.96 & 75.21 & 82.39 & 87.29 & 90.45 \\
\hline & $\mathrm{EGLS}_{3}$ & 10.57 & 21.91 & 47.13 & 62.91 & 75.60 & 85.43 & 90.31 & 95.66 \\
\hline & $\mathrm{HC} 0$ & 9.16 & 16.18 & 22.20 & 36.94 & 41.37 & 59.34 & 62.19 & 75.68 \\
\hline & $\mathrm{HC} 2$ & 8.95 & 15.74 & 21.71 & 36.44 & 41.06 & 59.19 & 62.09 & 75.71 \\
\hline & $\mathrm{HC} 3$ & 8.85 & 15.32 & 21.47 & 36.20 & 40.37 & 58.90 & 61.96 & 75.68 \\
\hline & $\mathrm{HC} 4$ & 8.23 & 14.93 & 21.14 & 35.86 & 39.91 & 58.78 & 62.03 & 75.64 \\
\hline & $\mathrm{HC} 4 \mathrm{~m}$ & 8.39 & 15.03 & 21.47 & 35.82 & 40.05 & 58.87 & 62.07 & 75.66 \\
\hline \multirow{8}{*}{$\chi_{(2)}^{2}$} & $\mathrm{EGLS}_{1}$ & 6.34 & 19.10 & 41.88 & 65.11 & 77.61 & 88.60 & 92.66 & 96.53 \\
\hline & $\mathrm{EGLS}_{2}$ & 6.04 & 18.06 & 37.82 & 56.39 & 68.02 & 77.38 & 83.09 & 86.02 \\
\hline & $\mathrm{EGLS}_{3}$ & 5.73 & 15.74 & 32.92 & 55.54 & 68.99 & 83.42 & 88.37 & 94.98 \\
\hline & $\mathrm{HC} 0$ & 5.80 & 12.34 & 16.82 & 34.64 & 39.82 & 59.45 & 56.42 & 77.04 \\
\hline & $\mathrm{HC} 2$ & 5.43 & 11.85 & 16.45 & 34.32 & 39.34 & 59.17 & 56.19 & 76.84 \\
\hline & $\mathrm{HC} 3$ & 5.18 & 11.43 & 16.42 & 33.94 & 38.94 & 58.83 & 55.78 & 76.62 \\
\hline & $\mathrm{HC} 4$ & 4.98 & 11.09 & 15.63 & 33.84 & 38.69 & 58.50 & 55.40 & 76.50 \\
\hline & $\mathrm{HC} 4 \mathrm{~m}$ & 4.93 & 11.04 & 15.92 & 33.90 & 38.67 & 58.50 & 55.47 & 76.52 \\
\hline
\end{tabular}


Table 4.8 Nonnull rejection rates $(\%)$, second simulation experiment; $\lambda \approx 6$.

\begin{tabular}{|c|c|c|c|c|c|c|c|c|c|}
\hline & $n$ & \multicolumn{2}{|c|}{50} & \multicolumn{2}{|c|}{100} & \multicolumn{2}{|c|}{150} & \multicolumn{2}{|c|}{200} \\
\hline$a$ & & $5 \%$ & $10 \%$ & $5 \%$ & $10 \%$ & $5 \%$ & $10 \%$ & $5 \%$ & $10 \%$ \\
\hline \multirow{8}{*}{1.0} & $\mathrm{EGLS}_{1}$ & 22.62 & 34.23 & 47.75 & 59.59 & 67.13 & 76.52 & 78.93 & 87.26 \\
\hline & $\mathrm{EGLS}_{2}$ & 22.62 & 34.23 & 47.75 & 59.58 & 67.13 & 76.52 & 78.93 & 87.26 \\
\hline & $\mathrm{EGLS}_{3}$ & 20.86 & 31.57 & 44.44 & 56.92 & 64.96 & 74.41 & 78.74 & 86.23 \\
\hline & $\mathrm{HCO}$ & 22.31 & 33.21 & 46.94 & 59.10 & 66.60 & 75.62 & 79.95 & 86.25 \\
\hline & $\mathrm{HC} 2$ & 22.38 & 33.15 & 46.94 & 59.18 & 66.61 & 75.62 & 79.95 & 86.26 \\
\hline & HC3 & 22.41 & 33.15 & 46.93 & 59.18 & 66.60 & 75.62 & 79.94 & 86.25 \\
\hline & $\mathrm{HC} 4$ & 22.39 & 33.14 & 46.84 & 59.21 & 66.60 & 75.64 & 79.94 & 86.24 \\
\hline & $\mathrm{HC} 4 \mathrm{~m}$ & 22.40 & 33.16 & 46.90 & 59.23 & 66.60 & 75.62 & 79.93 & 86.25 \\
\hline \multirow{8}{*}{2.5} & $\mathrm{EGLS}_{1}$ & 25.38 & 42.28 & 68.49 & 78.44 & 87.46 & 91.86 & 95.30 & 97.39 \\
\hline & $\mathrm{EGLS}_{2}$ & 25.38 & 42.28 & 68.49 & 78.44 & 87.46 & 91.86 & 95.30 & 97.39 \\
\hline & $\mathrm{EGLS}_{3}$ & 27.73 & 42.23 & 65.97 & 77.50 & 87.15 & 92.46 & 95.79 & 97.79 \\
\hline & $\mathrm{HCO}$ & 51.01 & 62.13 & 84.03 & 90.23 & 95.64 & 97.66 & 98.85 & 99.53 \\
\hline & $\mathrm{HC} 2$ & 47.79 & 59.07 & 82.81 & 89.47 & 95.31 & 97.51 & 98.84 & 99.50 \\
\hline & $\mathrm{HC} 3$ & 43.06 & 54.45 & 80.76 & 88.66 & 94.95 & 97.32 & 98.74 & 99.47 \\
\hline & $\mathrm{HC} 4$ & 32.17 & 42.53 & 76.20 & 85.28 & 93.55 & 96.73 & 98.57 & 99.34 \\
\hline & $\mathrm{HC} 4 \mathrm{~m}$ & 41.00 & 51.61 & 80.01 & 88.02 & 94.64 & 97.22 & 98.70 & 99.43 \\
\hline \multirow{8}{*}{3.5} & $\mathrm{EGLS}_{1}$ & 21.07 & 30.78 & 74.12 & 83.79 & 92.48 & 95.67 & 97.91 & 98.81 \\
\hline & $\mathrm{EGLS}_{2}$ & 21.02 & 30.75 & 74.16 & 83.81 & 92.49 & 95.68 & 97.91 & 98.81 \\
\hline & $\mathrm{EGLS}_{3}$ & 26.90 & 47.07 & 74.06 & 85.72 & 94.24 & 97.23 & 98.60 & 99.38 \\
\hline & $\mathrm{HC} 0$ & 73.61 & 83.11 & 96.05 & 98.27 & 99.57 & 99.83 & 99.99 & 100.00 \\
\hline & $\mathrm{HC} 2$ & 62.24 & 73.76 & 94.07 & 97.17 & 99.40 & 99.80 & 99.97 & 100.00 \\
\hline & $\mathrm{HC} 3$ & 47.73 & 59.95 & 91.19 & 95.81 & 98.93 & 99.74 & 99.96 & 100.00 \\
\hline & $\mathrm{HC} 4$ & 24.94 & 34.80 & 81.64 & 90.48 & 98.06 & 99.40 & 99.92 & 99.98 \\
\hline & $\mathrm{HC} 4 \mathrm{~m}$ & 41.28 & 51.96 & 88.93 & 94.80 & 98.77 & 99.66 & 99.96 & 100.00 \\
\hline \multirow{8}{*}{5.0} & $\mathrm{EGLS}_{1}$ & 41.01 & 45.25 & 66.76 & 83.94 & 94.59 & 97.09 & 98.71 & 99.27 \\
\hline & $\mathrm{EGLS}_{2}$ & 41.96 & 46.44 & 66.82 & 83.98 & 94.67 & 97.11 & 98.72 & 99.27 \\
\hline & $\mathrm{EGLS}_{3}$ & 43.89 & 61.13 & 76.91 & 91.51 & 97.12 & 99.18 & 99.62 & 99.90 \\
\hline & $\mathrm{HC} 0$ & 93.47 & 96.71 & 99.30 & 99.84 & 99.99 & 100.00 & 100.00 & 100.00 \\
\hline & $\mathrm{HC} 2$ & 74.15 & 82.47 & 97.94 & 99.44 & 99.97 & 100.00 & 100.00 & 100.00 \\
\hline & $\mathrm{HC} 3$ & 47.59 & 59.37 & 95.83 & 98.49 & 99.94 & 99.99 & 100.00 & 100.00 \\
\hline & $\mathrm{HC} 4$ & 20.31 & 33.75 & 86.83 & 94.42 & 99.76 & 99.97 & 100.00 & 100.00 \\
\hline & $\mathrm{HC} 4 \mathrm{~m}$ & 36.97 & 49.60 & 93.75 & 97.64 & 99.91 & 99.99 & 100.00 & 100.00 \\
\hline
\end{tabular}


Table 4.9 Nonnull rejection rates (\%), second simulation experiment; $\lambda \approx 26$.

\begin{tabular}{|c|c|c|c|c|c|c|c|c|c|}
\hline & $n$ & \multicolumn{2}{|c|}{50} & \multicolumn{2}{|c|}{100} & \multicolumn{2}{|c|}{150} & \multicolumn{2}{|c|}{200} \\
\hline$a$ & & $5 \%$ & $10 \%$ & $5 \%$ & $10 \%$ & $5 \%$ & $10 \%$ & $5 \%$ & $10 \%$ \\
\hline \multirow{8}{*}{1.0} & $\mathrm{EGLS}_{1}$ & 10.52 & 17.15 & 19.61 & 29.15 & 29.52 & 39.01 & 37.91 & 48.87 \\
\hline & $\mathrm{EGLS}_{2}$ & 10.52 & 17.15 & 19.61 & 29.15 & 29.52 & 39.01 & 37.90 & 48.87 \\
\hline & $\mathrm{EGLS}_{3}$ & 9.64 & 16.10 & 18.93 & 27.61 & 26.91 & 38.02 & 36.96 & 48.73 \\
\hline & $\mathrm{HC} 0$ & 10.25 & 16.82 & 19.04 & 27.89 & 27.11 & 36.59 & 35.40 & 46.71 \\
\hline & $\mathrm{HC} 2$ & 10.23 & 16.92 & 19.08 & 27.90 & 27.08 & 36.57 & 35.40 & 46.68 \\
\hline & $\mathrm{HC} 3$ & 10.19 & 16.85 & 19.12 & 27.90 & 27.07 & 36.55 & 35.40 & 46.67 \\
\hline & $\mathrm{HC} 4$ & 10.27 & 16.78 & 19.14 & 27.86 & 27.05 & 36.54 & 35.42 & 46.66 \\
\hline & $\mathrm{HC} 4 \mathrm{~m}$ & 10.30 & 16.81 & 19.12 & 27.86 & 27.04 & 36.53 & 35.39 & 46.64 \\
\hline \multirow{8}{*}{2.5} & $\mathrm{EGLS}_{1}$ & 12.79 & 21.50 & 25.01 & 34.58 & 33.35 & 45.09 & 44.58 & 55.22 \\
\hline & $\mathrm{EGLS}_{2}$ & 12.79 & 21.50 & 25.01 & 34.58 & 33.34 & 45.09 & 44.58 & 55.22 \\
\hline & $\mathrm{EGLS}_{3}$ & 15.39 & 24.77 & 33.59 & 44.04 & 47.51 & 59.18 & 62.71 & 71.72 \\
\hline & $\mathrm{HCO}$ & 32.63 & 42.59 & 57.70 & 66.40 & 73.36 & 79.90 & 83.74 & 88.90 \\
\hline & $\mathrm{HC} 2$ & 31.12 & 42.02 & 57.66 & 66.83 & 73.86 & 80.03 & 83.83 & 88.81 \\
\hline & $\mathrm{HC} 3$ & 29.46 & 40.23 & 57.84 & 66.67 & 73.77 & 80.01 & 83.81 & 88.86 \\
\hline & $\mathrm{HC} 4$ & 22.81 & 32.14 & 57.64 & 65.91 & 73.99 & 80.23 & 83.61 & 88.93 \\
\hline & $\mathrm{HC} 4 \mathrm{~m}$ & 28.18 & 38.63 & 58.19 & 66.72 & 73.63 & 80.10 & 83.88 & 88.84 \\
\hline \multirow{8}{*}{3.5} & $\mathrm{EGLS}_{1}$ & 14.07 & 23.14 & 29.06 & 38.31 & 37.56 & 49.12 & 48.40 & 58.29 \\
\hline & $\mathrm{EGLS}_{2}$ & 13.99 & 23.12 & 29.08 & 38.31 & 37.56 & 49.13 & 48.42 & 58.30 \\
\hline & $\mathrm{EGLS}_{3}$ & 21.76 & 34.18 & 50.32 & 61.75 & 68.55 & 77.91 & 83.48 & 88.63 \\
\hline & $\mathrm{HC} 0$ & 54.57 & 66.24 & 84.99 & 89.99 & 95.13 & 97.20 & 98.32 & 99.27 \\
\hline & $\mathrm{HC} 2$ & 48.96 & 60.04 & 83.92 & 89.12 & 94.74 & 96.90 & 98.25 & 99.16 \\
\hline & $\mathrm{HC} 3$ & 39.46 & 49.46 & 81.66 & 87.70 & 94.35 & 96.50 & 98.21 & 99.09 \\
\hline & $\mathrm{HC} 4$ & 21.28 & 30.32 & 74.19 & 82.51 & 92.70 & 95.64 & 97.91 & 98.90 \\
\hline & $\mathrm{HC} 4 \mathrm{~m}$ & 34.25 & 44.32 & 80.30 & 86.61 & 94.10 & 96.36 & 98.17 & 99.06 \\
\hline \multirow{8}{*}{5.0} & $\mathrm{EGLS}_{1}$ & 16.36 & 22.13 & 35.22 & 45.08 & 45.25 & 55.74 & 55.72 & 65.21 \\
\hline & $\mathrm{EGLS}_{2}$ & 16.78 & 22.23 & 35.20 & 45.14 & 45.33 & 55.79 & 55.77 & 65.26 \\
\hline & $\mathrm{EGLS}_{3}$ & 32.27 & 48.61 & 68.55 & 80.77 & 90.30 & 94.25 & 96.91 & 98.25 \\
\hline & $\mathrm{HCO}$ & 83.10 & 89.31 & 98.19 & 99.30 & 99.88 & 99.99 & 100.00 & 100.00 \\
\hline & $\mathrm{HC} 2$ & 63.53 & 73.10 & 96.81 & 98.56 & 99.86 & 99.94 & 100.00 & 100.00 \\
\hline & $\mathrm{HC} 3$ & 39.93 & 51.41 & 94.49 & 97.50 & 99.73 & 99.91 & 100.00 & 100.00 \\
\hline & $\mathrm{HC} 4$ & 17.27 & 28.42 & 84.89 & 93.28 & 99.24 & 99.82 & 99.99 & 100.00 \\
\hline & $\mathrm{HC} 4 \mathrm{~m}$ & 31.60 & 42.36 & 92.81 & 96.50 & 99.67 & 99.90 & 99.99 & 100.00 \\
\hline
\end{tabular}


Table 4.10 Nonnull rejection rates (\%), third simulation experiment; homoskedasticity and heteroskedasticity.

\begin{tabular}{|c|c|c|c|c|c|c|c|c|c|}
\hline & $n$ & \multicolumn{2}{|c|}{50} & \multicolumn{2}{c|}{100} & \multicolumn{2}{c|}{150} & \multicolumn{2}{c|}{200} \\
\hline$\lambda$ & & $5 \%$ & $10 \%$ & $5 \%$ & $10 \%$ & $5 \%$ & $10 \%$ & $5 \%$ & $10 \%$ \\
\hline \multirow{6}{*}{1.00} & EGLS $_{1}$ & 11.77 & 23.75 & 55.29 & 68.80 & 78.17 & 86.82 & 91.59 & 95.83 \\
& EGLS $_{2}$ & 11.83 & 23.75 & 55.29 & 68.76 & 78.20 & 86.83 & 91.59 & 95.83 \\
& EGLS $_{3}$ & 23.07 & 33.98 & 49.81 & 64.15 & 70.90 & 81.51 & 87.37 & 93.40 \\
& HC0 & 34.39 & 46.31 & 65.83 & 76.44 & 81.85 & 89.21 & 93.41 & 96.61 \\
& HC2 & 30.53 & 43.25 & 64.53 & 75.02 & 80.69 & 88.62 & 93.11 & 96.39 \\
& HC3 & 26.86 & 38.03 & 62.19 & 72.90 & 79.24 & 87.76 & 92.64 & 96.19 \\
& HC4 & 18.09 & 26.04 & 54.73 & 66.98 & 75.09 & 85.48 & 91.64 & 95.39 \\
& HC4m $_{1}$ & 24.72 & 34.40 & 60.09 & 71.76 & 78.43 & 87.20 & 92.40 & 96.07 \\
\hline \multirow{6}{*}{2.44} & EGLS & 23.84 & 40.59 & 84.30 & 92.65 & 97.74 & 99.16 & 99.81 & 99.93 \\
& EGLS & 23.99 & 40.83 & 84.31 & 92.65 & 97.74 & 99.16 & 99.81 & 99.93 \\
& EGLS & 41.48 & 56.27 & 79.63 & 89.52 & 95.38 & 98.30 & 99.45 & 99.88 \\
& HC0 & 60.56 & 72.30 & 90.71 & 94.95 & 97.95 & 99.16 & 99.80 & 99.93 \\
& HC2 & 54.47 & 66.00 & 88.25 & 93.38 & 97.37 & 98.96 & 99.74 & 99.90 \\
& HC3 & 45.38 & 57.37 & 85.23 & 91.39 & 96.43 & 98.68 & 99.71 & 99.89 \\
& HC4 & 28.07 & 37.05 & 76.10 & 85.98 & 93.65 & 97.68 & 99.53 & 99.83 \\
& HC4m & 40.44 & 52.17 & 83.38 & 90.38 & 95.90 & 98.44 & 99.66 & 99.87 \\
\hline
\end{tabular}

Table 4.12 contains the mean values of the different variance estimators. First, note that the $\mathrm{HC} 0$ estimator is very optimistic when the data are leveraged, i.e., it substantially underestimates the true variance. Second, HC2 is the least biased heteroskedasticity-consistent estimator. Third, $\mathrm{HC} 4$ and $\mathrm{HC} 4 \mathrm{~m}$ can be quite positively biased, especially $\mathrm{HC} 4$; that is, they tend to overestimate the true variance, more so when the data are leveraged and the sample size is small $(n=50)$.

A valid question that is commonly posed is: To what extent is it worthwhile to base inferences on the OLSE coupled with heteroskedasticity-robust standard errors under unequal error variances instead of incorporating a dispersion submodel to the regression model and then carrying out estimation and performing inferences using EGLS? The results in Table 4.11 show that the EGLS estimator of the regression parameters can be considerably more accurate than the OLS estimator, especially when the data are not leveraged. The results in Table 4.12 show that the standard, baseline heteroskedasticity-robust standard error ( $\mathrm{HC} 0)$ tends to be largely negatively biased when the data are leveraged, thus resulting in liberal quasi- $t$ testing inferences. Better control of the type I error frequency is achieved by using a positively biased heteroskedasticity-robust standard error, such as $\mathrm{HC} 3$ and $\mathrm{HC} 4 \mathrm{~m}$.

We then arrive at the following relevant question: How should estimated variances be evaluated? Simonoff (1993) argues that since they are primarily used for performing inferences, a comparison of different variance estimators should involve their intended use. In this dissertation, we focus on the accuracy of testing inferences based on statistics that employ different standard errors. 
Table 4.11 True variances of $\hat{\beta}_{2}$ and $\tilde{\beta}_{2}$.

\begin{tabular}{c|c|c|cc}
\hline$\lambda$ & $a$ & $n$ & $\operatorname{var}\left(\hat{\beta}_{2}\right)$ & $\operatorname{var}\left(\tilde{\beta}_{2}\right)$ \\
\hline \multirow{4}{*}{5.16} & 1.0 & 50 & 0.4930 & 0.3938 \\
& & 200 & 0.1233 & 0.0985 \\
& 5.0 & 50 & 0.0566 & 0.0534 \\
& & 200 & 0.0141 & 0.0133 \\
\hline \multirow{3}{*}{25.18} & 1.0 & 50 & 1.4085 & 0.5842 \\
& & 200 & 0.3521 & 0.1461 \\
& 5.0 & 50 & 0.0884 & 0.0665 \\
& & 200 & 0.0221 & 0.0166 \\
\hline
\end{tabular}

Table 4.12 Mean estimated variances of $\hat{\beta}_{2}$ and $\tilde{\beta}_{2}$.

\begin{tabular}{c|c|c|cccccc}
\hline$\lambda$ & $a$ & $n$ & $\mathrm{HC} 0$ & $\mathrm{HC} 2$ & $\mathrm{HC} 3$ & $\mathrm{HC} 4$ & $\mathrm{HC} 4 \mathrm{~m}$ & EGLS $_{2}$ \\
\hline \multirow{3}{*}{5.16} & 1.0 & 50 & 0.4638 & 0.4917 & 0.5215 & 0.5071 & 0.5329 & 0.4003 \\
& & 200 & 0.1213 & 0.1231 & 0.1248 & 0.1240 & 0.1255 & 0.1052 \\
& 5.0 & 50 & 0.0265 & 0.0860 & 0.4411 & 15.3550 & 1.0580 & 0.1294 \\
& & 200 & 0.0121 & 0.0144 & 0.0172 & 0.0252 & 0.0188 & 0.0299 \\
\hline \multirow{3}{*}{25.18} & 1.0 & 50 & 1.3171 & 1.3977 & 1.4835 & 1.4414 & 1.5178 & 0.8616 \\
& & 200 & 0.3461 & 0.3511 & 0.3563 & 0.3537 & 0.3582 & 0.2311 \\
& 5.0 & 50 & 0.0705 & 0.2396 & 1.2501 & 43.7030 & 3.0062 & 0.6396 \\
& & 200 & 0.0209 & 0.0239 & 0.0278 & 0.0385 & 0.0299 & 0.1889 \\
\hline
\end{tabular}




\section{Empirical applications}

In what follows we shall present and discuss two empirical applications. Testing inferences shall be performed using $z$ and quasi- $t$ tests. Unless stated otherwise, the variables used in the skedastic function for EGLS estimation are the same as those used to model the mean response.

\subsection{Man-hours for manning installations data}

The dependent variable $(y)$ is the number of monthly man-hours for manning installations in the U.S. Navy in Bachelor Office Quarters, and the independent variables are average daily occupancy $\left(x_{2}\right)$ and number of building wings $\left(x_{3}\right)$. The source of the data is Myers (1990, Table 5.2, p. 218). The data contain 25 observations on the response and on the covariates. Observations 22 and 23 appear to be leverage data points: $h_{22}=0.720$ and $h_{23}=0.877$, whereas $3 p / n=0.360$. The regression model we use is

$$
y_{i}=\beta_{1}+\beta_{2} x_{i 2}+\beta_{3} x_{i 3}+\epsilon_{i}
$$

$i=1, \ldots, 25$.

Table 5.1 contains some descriptive statistics on the response variable $(y)$ and on the independents variables. We note that the distribution of $y$ seems to be positively asymmetrically distributed. The response minimal and maximal values are 164.38 and 8266.77 , respectively, and its average value equals 2109.39.

Table 5.1 Descriptive Statistics, complete data; man-hours for manning installations data.

\begin{tabular}{c|c|c|c}
\hline Statistics & $y$ & $x_{2}$ & $x_{3}$ \\
\hline Minimum & 164.38 & 2.00 & 1.00 \\
Maximum & 8266.77 & 811.08 & 58.00 \\
Mean & 2109.39 & 117.16 & 11.12 \\
Median & 1845.89 & 95.00 & 9.00 \\
Variance & 3787883.00 & 28203.08 & 145.03 \\
Standard deviation & 1946.25 & 167.94 & 12.04 \\
Skewness & 1.46 & 3.06 & 2.49 \\
Kurtosis & 2.21 & 9.92 & 7.26 \\
\hline
\end{tabular}

We tested for the presence of heteroskedasticity using the test proposed by Koenker (1981). The null hypothesis of equal error variances was rejected at the 5\% significance level. Hence, there is evidence that the data are heteroskedastic. 
Next, the parameters of Model (5.1) were estimated by ordinary and estimated generalized least squares. The OLS point estimates are $\hat{\beta}_{1}=618.551, \hat{\beta}_{2}=4.045$ and $\hat{\beta}_{3}=91.455$; the EGLS ${ }_{1}$ point estimates are $\tilde{\tilde{\beta}}_{1}=125.598, \tilde{\tilde{\beta}}_{2}=22.570$ and $\tilde{\tilde{\beta}}_{3}=-6.954$; the EGLS point estimates are $\tilde{\beta}_{1}=125.580, \tilde{\beta}_{2}=22.570$ and $\tilde{\beta}_{3}=-6.951$; the EGLS 3 point estimates are $\hat{\beta}_{1}=132.358, \hat{\beta}_{2}=18.778$ and $\hat{\beta}_{3}=22.207$. It is noteworthy that the OLS parameter estimates are considerably different than those obtained with the three EGLS strategies. We also note that the EGLS ${ }_{1}$ and EGLS $S_{2}$ estimates of $\beta_{3}$ are quite different from the corresponding EGLS $_{3}$ estimate; the first two are negative wehereas the latter is postive. Table 5.2 presents the standard errors of the three parameter estimates $\left(\mathrm{se}_{i}, i=1, \ldots, 3\right)$ obtained using the different approaches. The largest standard errors are those obtained using the HC4 covariance matrix estimator followed by $\mathrm{HC} 4 \mathrm{~m}$. Overall, the smallest standard errors are EGLS ${ }_{1}$ and EGLS 2 .

Table 5.2 Standard errors, complete data; man-hours for manning installations data.

\begin{tabular}{c|c|c|c}
\hline & $\mathrm{se}_{1}$ & $\mathrm{se}_{2}$ & $\mathrm{se}_{3}$ \\
\hline EGLS $_{1}$ & 81.47 & 1.87 & 12.01 \\
EGLS $_{2}$ & 81.47 & 1.87 & 12.01 \\
EGLS $_{3}$ & 119.86 & 2.73 & 27.12 \\
$\mathrm{HC} 0$ & 236.44 & 2.76 & 28.92 \\
$\mathrm{HC} 2$ & 372.38 & 7.11 & 59.12 \\
$\mathrm{HC} 3$ & 741.83 & 19.90 & 136.66 \\
$\mathrm{HC} 4$ & 4448.37 & 161.34 & 926.48 \\
$\mathrm{HC} 4 \mathrm{~m}$ & 1115.32 & 33.53 & 215.30 \\
OLS & 345.48 & 1.71 & 23.85 \\
\hline
\end{tabular}

Consider the test of $\mathcal{H}_{0}: \beta_{3}=0$ against $\mathcal{H}_{1}: \beta_{3} \neq 0$. The interest lies in determining whether $x_{3}$ should be removed from the regression model given that $x_{2}$ is already in the model. Table 5.3 contains the tests $p$-values for the complete data and for the data without the two leverage points. When all observations are used, the only tests that yield rejection of the null hypothesis at the usual significance levels are the test whose statistic uses OLS standard error and the $\mathrm{HCO}$ test. It is interesting to note that when the two leverage data points are removed from the data all tests yield $p$-values in excess of 0.5 , thus indicating that the evidence against the null hypothesis is quite small. Table 5.4 contains the parameter estimates we obtained after the withdrawal of the identified leverage points from the data. Note that now all estimates of $\beta_{3}$ are positive. Table 5.5 contains the standard errors obtained after the withdrawal of the identified leverage points. Note that there is now less discrepancy in these quantities among the estimators used. The HC4 and HC4m standard errors are still the largest ones and the EGLS standard errors are typically the smallest ones.

We note that rejection of the null hypothesis is driven by only two atypical data points. When such observations are not in the data, the EGLS $1, \mathrm{EGLS}_{2}, \mathrm{EGLS}_{3}, \mathrm{HC} 2, \mathrm{HC} 3, \mathrm{HC} 4$ and $\mathrm{HC} 4 \mathrm{~m}$ tests lead to the same conclusion, namely: the covariate $x_{3}$ can be safely removed from the regression model. Indeed, the coefficient of determination for the regression with the incomplete data equals 0.959477 ; when $x_{3}$ is removed from the model $R^{2}$ remains nearly unchanged: 0.959463 . This is evidence that, as long as the two leverage points are not in the 
Table 5.3 Tests $p$-values; man-hours for manning installations data.

\begin{tabular}{lrlr}
\hline \multicolumn{2}{l}{ complete data, $n=25$} & \multicolumn{2}{l}{ incomplete data, $n=23$} \\
\hline Test & $p$-value & Test & $p$-value \\
\hline EGLS $_{1}$ & 0.56266 & EGLS $_{1}$ & 0.81127 \\
EGLS $_{2}$ & 0.56288 & EGLS $_{2}$ & 0.81218 \\
EGLS $_{3}$ & 0.41285 & EGLS $_{3}$ & 0.53989 \\
HC0 & 0.00157 & HC0 & 0.92456 \\
HC2 & 0.12185 & HC2 & 0.93266 \\
HC3 & 0.50337 & HC3 & 0.94074 \\
HC4 & 0.92137 & HC4 & 0.94626 \\
HC4m & 0.67100 & HC4m & 0.94358 \\
OLS & 0.00013 & OLS & 0.93363 \\
\hline
\end{tabular}

Table 5.4 Parameter estimates, incomplete data; man-hours for manning installations data.

\begin{tabular}{c|c|c|c}
\hline & $\beta_{1}$ & $\beta_{2}$ & $\beta_{3}$ \\
\hline OLS & 56.538 & 22.802 & 1.666 \\
EGLS $_{1}$ & 85.837 & 22.348 & 1.851 \\
EGLS $_{2}$ & 85.860 & 22.354 & 1.824 \\
EGLS $_{3}$ & 41.784 & 22.770 & 4.601 \\
\hline
\end{tabular}

Table 5.5 Standard errors, incomplete data; man-hours for manning installations data.

\begin{tabular}{c|c|c|c}
\hline & $\mathrm{se}_{1}$ & $\mathrm{se}_{2}$ & $\mathrm{se}_{3}$ \\
\hline EGLS $_{1}$ & 63.16 & 1.65 & 7.75 \\
EGLS $_{2}$ & 62.86 & 1.65 & 7.67 \\
EGLS $_{3}$ & 74.47 & 1.16 & 7.51 \\
$\mathrm{HC} 0$ & 88.84 & 1.39 & 17.59 \\
$\mathrm{HC} 2$ & 97.08 & 1.53 & 19.71 \\
$\mathrm{HC} 3$ & 107.61 & 1.74 & 22.40 \\
$\mathrm{HC} 4$ & 119.23 & 2.55 & 24.71 \\
$\mathrm{HC} 4 m$ & 110.91 & 1.85 & 23.53 \\
OLS & 131.74 & 1.80 & 20.00 \\
\hline
\end{tabular}

dataset, the second covariate does not significantly add to the fit. The HC2 through HC4m quasi$t$ tests and the EGLS tests deliver the correct inference in both scenarios. Such inference is not driven by only two (atypical) data points. The HCO and OLS testing inferences, in contrast, are driven by only two observations: when the two atypical points are in the data the HCO and OLS tests lead the practitioner to incorrectly conclude that $x_{3}$ must be in the model.

\subsection{Per capita spending on public education}

The data we shall now use were previously analyzed by Cribari-Neto (2004). The source of the data is the U.S. Department of Commerce. The response (y) is per capita spending on public 
schools and the covariates, $x$ and $x^{2}$, are per capita income by state in 1979 in the United States and its square; income is scaled by $10^{-4}$. The observation relative to Wisconsin is missing, and that for Washington, D.C. is included. Thus, $n=50$. The regression model is

$$
y_{i}=\beta_{1}+\beta_{2} x_{i}+\beta_{3} x_{i}^{2}+\epsilon_{i}
$$

$i=1, \ldots, 50$.

Table 5.6 contains some descriptive statistics on the response variable $(y)$ and on the independent variable $(x)$. We note that there is positive asymmetry in the dependent variable $(y)$. Additionally, the its minimal and maximal values are 259 and 821, respectively.

Table 5.6 Descriptive Statistics, complete data; per capita spending on public education data.

\begin{tabular}{c|c|c}
\hline Statistics & $y$ & $x$ \\
\hline Minimum & 259.00 & 0.57 \\
Maximum & 821.00 & 1.08 \\
Mean & 373.26 & 0.76 \\
Median & 354.00 & 0.76 \\
Variance & 8940.32 & 0.01 \\
Standard deviation & 94.55 & 0.10 \\
Skewness & 2.16 & 0.66 \\
Kurtosis & 8.08 & 0.46 \\
\hline
\end{tabular}

The Koenker (1981) test suggests that there is heteroskedasticity. The OLS parameter estimates are $\hat{\beta}_{1}=834.254, \hat{\beta}_{2}=-1837.172$ and $\hat{\beta}_{3}=1588.499$; the EGLS 1 estimates are

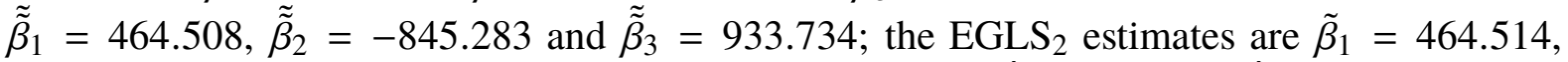

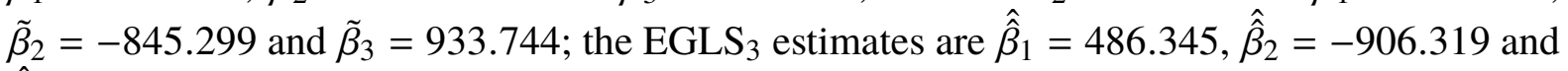
$\hat{\hat{\beta}}_{3}=975.892$. Table 5.7 contains the standard errors of the three parameter estimates obtained using the different approaches. Again, the largest standard errors are those obtained using the $\mathrm{HC} 4$ covariance matrix estimator followed by $\mathrm{HC} 4 \mathrm{~m}$. The smallest standard errors are obtained by OLS followed by the EGLS ones.

The interest lies in testing a linear versus a quadratic functional form, i.e., in testing $\mathcal{H}_{0}$ : $\beta_{3}=0$ against $\mathcal{H}_{1}: \beta_{3} \neq 0$. Table 5.8 contains the tests $p$-values. Notice that the OLS and $\mathrm{HC} 0$ tests reject the null hypothesis (in favor of a quadratic model specification) at the $10 \%$ significance level. The data are plotted in Figure 5.1. It is clear that Alaska is a leverage and atypical data point. Indeed, its leverage value $\left(h_{\text {Alaska }}\right)$ equals 0.651 which considerably exceeds three times the mean leverage $(3 p / n=0.180)$. Considering this reference value $(0.180)$, two other observations are singled out as leverage points, namely: Washington, D.C. $\left(h_{\text {D.C. }}=0.208\right)$ and Mississippi $\left(h_{\text {Mississippi }}=0.200\right)$. We shall, however, follow Cribari-Neto (2004) and focus on the impact of the most atypical observation (Alaska) on the resulting inferences. We removed Alaska from the data, estimated the parameters and performed the OLS, EGLS and quasi- $t$ tests again. We report the tests $p$-values in Table 5.8 ('incomplete data'). It is noteworthy that when Alaska is not in the data, except for EGLS ${ }_{1}$ and EGLS $S_{2}$, the tests $p$-values are large, and hence the null hypothesis is not rejected. The null hypothesis is marginally rejected at the $10 \%$ nominal level, however, by the EGLS ${ }_{1}$ and EGLS 2 tests. Hence, rejection of the null hypothesis by the 
OLS and $\mathrm{HC} 0$ tests with the complete data seems to be driven by a single atypical observation. In contrast, one does not reject the null hypothesis at the usual significance levels when using the $\mathrm{HC} 2, \mathrm{HC} 3, \mathrm{HC} 4$ and $\mathrm{HC} 4 \mathrm{~m}$ tests even when the data contain a strong leverage point, i.e., such testing inference is not substantially affected by an atypical observation. We note that the coefficient of determination $\left(R^{2}\right)$ for the regression with the incomplete data equals 0.501079 ; when $x^{2}$ is removed from the model it remains nearly unchanged: 0.498841 . This can be viewed as evidence that, as long as Alaska is not in the data, squared per capita income $\left(x^{2}\right)$ does not significantly add to the fit. The $\mathrm{EGLS}_{3} z$ test and the HC2 through HC4m quasi- $t$ tests deliver the correct inference in both scenarios. For instance, the HC3 (HC4) [HC4m] $p$-values computed using the complete and incomplete datasets are 0.4262 and $0.7747(0.7722$ and 0.8914) [0.5340 and 0.8096], respectively. The corresponding EGLS (EGLS $\left._{2}\right)$ [EGLS 3 ] $p$-values are 0.2082 and 0.0968 (0.2082 and 0.0971) [0.2336 and 0.2269]; notice the reduction in $p$-values when Alaska is removed from the data which contrasts to the sizable increased in the quasi- $t$ tests $p$-values. Table 5.9 contains the parameter estimates obtained after the withdrawal of Alaska from the data. Table 5.10 contains the standard errors obtained after the withdrawal of the outlying observation. Note that even though such figure become smaller, there is still considerable discrepancy among them. The $\mathrm{HC} 4$ and $\mathrm{HC} 4 \mathrm{~m}$ standard errors are still the largest ones and the EGLS standard errors are the smallest ones.

Table 5.7 Standard errors, complete data; per capita spending on public education data.

\begin{tabular}{c|c|c|c}
\hline & $\mathrm{se}_{1}$ & $\mathrm{se}_{2}$ & $\mathrm{se}_{3}$ \\
\hline EGLS $_{1}$ & 421.78 & 1123.43 & 741.90 \\
EGLS $_{2}$ & 421.77 & 1123.41 & 741.89 \\
EGLS $_{3}$ & 455.78 & 1226.69 & 819.24 \\
$\mathrm{HC} 0$ & 461.57 & 1244.67 & 830.90 \\
$\mathrm{HC} 2$ & 689.37 & 1868.52 & 1251.33 \\
$\mathrm{HC} 3$ & 1096.00 & 2977.71 & 1996.45 \\
$\mathrm{HC} 4$ & 3007.83 & 8181.65 & 5487.08 \\
$\mathrm{HC} 4 m$ & 1401.03 & 3808.81 & 2554.33 \\
OLS & 327.46 & 829.43 & 519.34 \\
\hline
\end{tabular}

It is interesting to note that the EGLS tests yielded conflicting inferences when the incomplete data were used: the EGLS 1 and EGLS 2 z tests rejected the null hypothesis (in favor of the quadratic specification) at the $10 \%$ significance level whereas the null hypothesis was not rejected by the EGLS $3 z$ test. Since the atypical observation (Alaska) is not in the data, one would expect the tests to not reject the null hypothesis under evaluation. The rejection of $\mathcal{H}_{0}$ by two EGLS tests when the outlier-free data were used was not expected. In order to further investigate that, we redid the analysis using a different skedastic function, namely:

$$
\sigma_{i}^{2}=\exp \left(\gamma_{1}+\gamma_{2} x_{i}\right)
$$

i.e., income squared, which is used as a mean regressor, is no longer used a dispersion regressor. The EGLS tests $p$-values computed using both complete and incomplete data using the new 
Table 5.8 Tests $p$-values; per capita spending on public education data.

\begin{tabular}{lrlr}
\hline \multicolumn{2}{l}{ complete data, $n=50$} & \multicolumn{2}{l}{ incomplete data, $n=49$} \\
\hline Test & $p$-value & Test & $p$-value \\
\hline EGLS $_{1}$ & 0.2082 & EGLS $_{1}$ & 0.0968 \\
EGLS $_{2}$ & 0.2082 & EGLS $_{2}$ & 0.0971 \\
EGLS $_{3}$ & 0.2336 & EGLS $_{3}$ & 0.2269 \\
HC0 & 0.0559 & HC0 & 0.6157 \\
HC2 & 0.2043 & HC2 & 0.6954 \\
HC3 & 0.4262 & HC3 & 0.7747 \\
HC4 & 0.7722 & HC4 & 0.8914 \\
HC4m & 0.5340 & HC4m & 0.8096 \\
OLS & 0.0022 & OLS & 0.6496 \\
\hline
\end{tabular}

Table 5.9 Parameter estimates, incomplete data; per capita spending on public education data.

\begin{tabular}{c|c|c|c}
\hline & $\beta_{1}$ & $\beta_{2}$ & $\beta_{3}$ \\
\hline OLS & -208.724 & 1000.016 & -314.051 \\
EGLS $_{1}$ & -426.827 & 1625.700 & -752.356 \\
EGLS $_{2}$ & -426.675 & 1625.271 & -752.061 \\
EGLS $_{3}$ & -362.640 & 1450.299 & -635.319 \\
\hline
\end{tabular}

Table 5.10 Standard errors, incomplete data; per capita spending on public education data.

\begin{tabular}{c|c|c|c}
\hline & $\mathrm{se}_{1}$ & $\mathrm{se}_{2}$ & $\mathrm{se}_{3}$ \\
\hline EGLS $_{1}$ & 268.82 & 704.97 & 453.13 \\
EGLS $_{2}$ & 268.89 & 705.18 & 453.27 \\
EGLS $_{3}$ & 305.18 & 808.19 & 525.71 \\
$\mathrm{HC} 0$ & 345.36 & 935.68 & 625.66 \\
$\mathrm{HC} 2$ & 437.03 & 1191.57 & 802.07 \\
$\mathrm{HC} 3$ & 591.91 & 1621.86 & 1097.14 \\
$\mathrm{HC} 4$ & 1228.88 & 3383.72 & 2299.70 \\
$\mathrm{HC} 4 m$ & 700.56 & 1923.43 & 1303.74 \\
OLS & 405.27 & 1064.06 & 691.30 \\
\hline
\end{tabular}

skedastic function are presented in Table 5.11. At the outset, note that the EGLS tests now deliver the same inferences in both scenarios; there is no longer contradiction between them. It is also noteworthy that all three $z$ tests now seem to deliver the correct inference when the incomplete data are used: the null hypothesis is not rejected (the three $p$-values are quite large), i.e., there is no evidence against the linear specification. However, when the complete data are used (Alaska is included in the data), the three EGLS tests yield rejection of the null hypothesis at the $10 \%$ significance level. Such inference (rejection of the linear specification in favor of the quadratic specification) seems to be driven by a single, atypical data point.

This empirical application shows that testing inferences reached after EGLS parameter estimation can be highly dependent on how the response variances are modeled. It also shows 


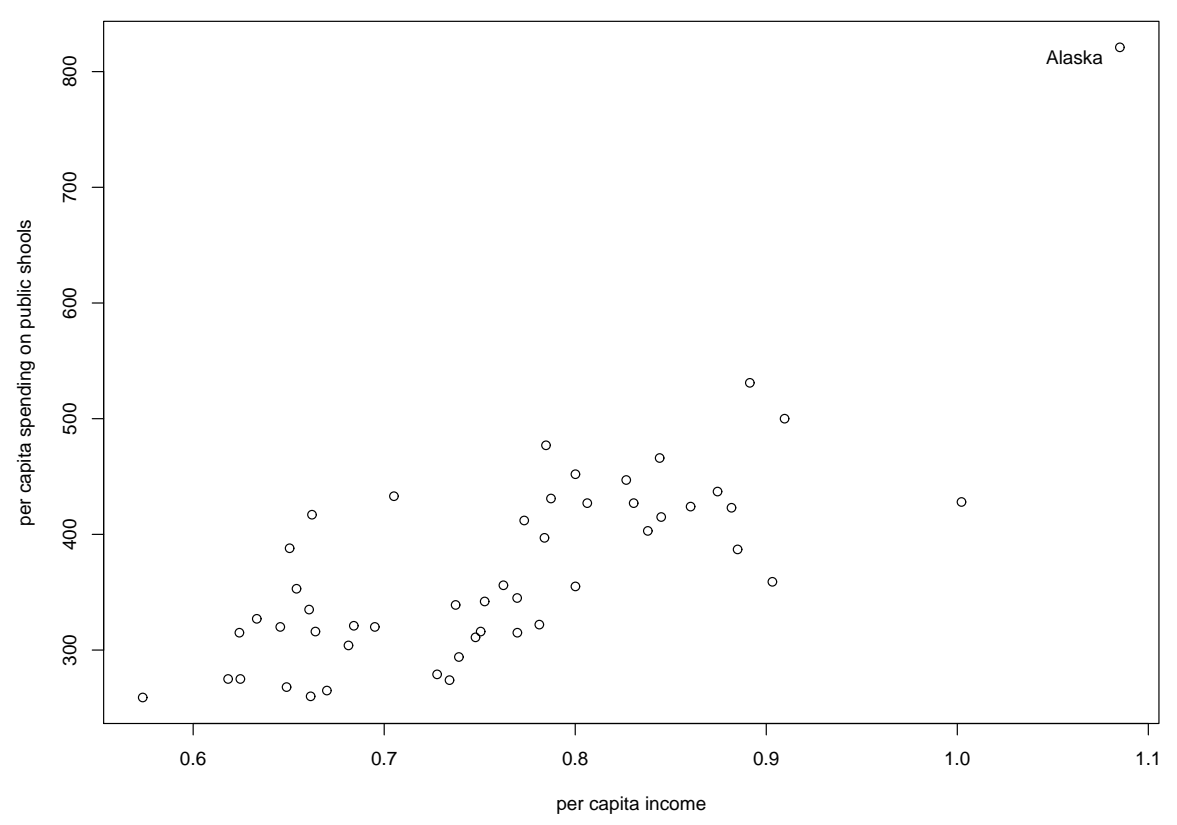

Figure 5.1 Per capita spending on public schools vs. per capita income $(\times 10,000)$.

Table 5.11 Tests $p$-values; per capita spending on public education data; alternative skedastic function.

\begin{tabular}{lrlr}
\hline \multicolumn{2}{l}{ complete data, $n=50$} & \multicolumn{3}{l}{ incomplete data, $n=49$} \\
\hline Test & $p$-value & Test & $p$-value \\
\hline EGLS $_{1}$ & 0.0738 & EGLS $_{1}$ & 0.8733 \\
EGLS $_{2}$ & 0.0738 & EGLS $_{2}$ & 0.8733 \\
EGLS $_{3}$ & 0.0706 & EGLS $_{3}$ & 0.9954 \\
\hline
\end{tabular}

that EGLS testing inferences can be quite affected by a single, atypical observation. The same happened, we saw earlier, with the $\mathrm{HCO}$ quasi- $t$ test. The other quasi- $t$ tests, however, proved reliable even under highly leveraged data. 


\section{Concluding remarks}

The linear regression model is commonly used in empirical analyses in many fields. Oftentimes such analyses employ cross sectional data and there is heteroskedasticity, i.e., the error variances are not the same for all observations in the sample. Practitioners frequently want to perform testing inferences on the model parameters, e.g., to decide which covariates should be included in the model and which covariates should be removed from it. Broadly speaking, two different testing strategies can be employed. The first involves using quasi- $t$ test statistics that are based on standard errors obtained from a heteroskedasticity-consistent covariance matrix estimator. These standard errors are asymptotically correct under both homoskedasticity and heteroskedasticity of unknown form. The second strategy requires expanding the model to model not only the mean effects but also the dispersion effects. In other words, it is necessary to specify a submodel for the response mean and a separate submodel for the response variances. The likelihood of model misspecification is thus increased. Which testing strategy should be preferred? We seeked to provide this question with an answer. Some authors in the literature focused on the accuracy of estimated variances and covariances of alternative parameter estimators, namely: ordinary least squares and estimated generalized least squares. According to Simonoff (1993), however, since estimated variances are mainly used for performing inferences, a comparison of different variance estimators should involve their intended use, such as the empirical coverages of associated confidence intervals and the sizes of associated tests. This is the approach we pursued. We compared the accuracy of testing inferences made using quasi- $t$ (ordinary least squares estimation coupled with asymptotically correct standard errors) and $z$ (estimated generalized least squares) tests.

Our numerical evaluations were performed using different models, different sample sizes, and different heteroskedasticity strengths. We evaluated the finite sample performances of several quasi- $t$ tests (i.e., of tests based on different heteroskedasticity-robust standard errors) and also those of standard $z$ tests based on estimated generalized least squares estimation. The main difference between the two approaches lies in the fact that the former only requires modeling of mean effects whereas the latter requires the practitioner to model both mean and dispersion effects. We also numerically evaluated the accuracy of point estimates obtained using the two estimation approaches. Our numerical results showed that point estimates obtained by estimated generalized least squares can be considerably more accurate than ordinary least squares point estimates when the data do not include leverage points, but the difference in accuracy becomes much smaller under leveraged data. As noted earlier, nonetheless, our chief interest lies in testing inferences. The numerical evidence we reported showed that overall the best performing quasi- $t$ tests are those based on $\mathrm{HC} 3$ and $\mathrm{HC} 4 \mathrm{~m}$ heteroskedasticity-robust standard errors. It 
was also shown that such tests typically display better control of the type I error frequency than $z$ tests carried out after joint mean and dispersion modeling, especially under leveraged data. For instance, in an extreme case of data containing a very atypical data point in one of our simulations, when the sample contained only 50 observations and the tests were carried out at the 5\% significance level, the EGLS 2 test null rejection rate exceeded $35 \%$ (i.e., it was more than seven times larger than the test significance level); the $\mathrm{HC} 3$ and $\mathrm{HC} 4 \mathrm{~m}$ null rejection rates were $5.06 \%$ and $3.24 \%$, respectively.

We have also presented and discussed two empirical applications. In one them (the empirical applications mentioned in the dissertation introduction), the interest lied in distinguishing between a linear and a quadratic model specification. In this application, the most reliable quasi- $t$ tests delivered what seems to be the correct inference, regardless of whether the data contain a leverage point which is also an outlier. In contrast, EGLS testing inferences proved to be highly dependent on how the responses variances are modeled and were also quite sensitive to a single, atypical observation (Alaska). This highlights an advantage of quasi- $t$ tests: they do not require the practitioner to model dispersion effects.

In future work, we shall address the following issues:

- We shall consider alternative covariance matrix estimators that are consistent under both homoskedasticity and heteroskedasticity, such as HC5 (Cribari-Neto, Souza, and Vasconcellos 2007) and HC5m (Li et al. 2016).

- We shall consider testing inferences on a vector of parameters rather than on a single model parameter. 


\section{References}

Atkinson, A. C., M. Riani, and F. Torti (2016). Robust methods for heteroskedastic regression. Computational Statistics and Data Analysis 104, 209-222.

Chesher, A. and I. Jewitt (1987). The bias of a heteroskedasticity consistent covariance matrix estimator. Econometrica 55, 1217-1222.

Cribari-Neto, F. (2004). Asymptotic inference under heteroskedasticity of unknown form. Computational Statistics and Data Analysis 45, 215-233.

Cribari-Neto, F. and M. G. A. Lima (2009). Heteroskedasticity-consistent interval estimators. Journal of Statistical Computation and Simulation 79, 787-803.

Cribari-Neto, F. and M. G. A. Lima (2010). Approximate inference in heteroskedastic regressions: A numerical evaluation. Journal of Applied Statistics 37(4), 591-615.

Cribari-Neto, F. and W. B. Silva (2011). A new heteroskedasticity-consistent covariance matrix estimator for the linear regression model. Advances in Statistical Analysis 95, 129-146.

Cribari-Neto, F., T. C. Souza, and K. L. P. Vasconcellos (2007). Inference under heteroskedasticity and leveraged data. Communications in Statistics - Theory and Methods 36. Errata: 37, 2008, 3329-3330., 1877-1888. Errata: 37, 2008, 3329-3330.

Doornik, J. A. (2009). An Object-Oriented Matrix Language Ox 6. London: Timberlake Consultants Press.

Harvey, A. C. (1976). Estimating regression models with multiplicative heteroscedasticity. Econometrica 44(3), 461-465.

Horn, S. D., R. A. Horn, and D. B. Duncan (1975). Estimating heteroskedastic variances in linear models. Journal of the American Statistical Association 70, 380-385.

Koenker, R. (1981). A note on Studentizing a test for heteroscedasticity. Journal of Econometrics 17, 107-112.

Lamport, L. (1986). ETEX: A Document Preparation System. Addison-Wesley Publishing Company.

Li, Shunyong et al. (2016). A new heteroskedasticity-consistent covariance matrix estimator and inference under heteroskedasticity. Journal of Statistical Computation and Simulation 87, $198-210$.

Long, J. S. and L. H. Ervin (2000). Using heteroskedasticity-consistent standard errors in the linear regression model. American Statistician 54(3), 217-224.

MacKinnon, J. G. and H. White (1985). Some heteroskedasticity-consistent covariance matrix estimators with improved finite-sample properties. Journal of Econometrics 29, 305-325.

Myers, R. H. (1990). Classical and Modern Regression with Applications. Belmont: Duxbury Press. 
R Core Team (2016). R: A Language and Environment for Statistical Computing. R Foundation for Statistical Computing. Vienna, Austria. URL: https://www.R-project .org/.

Simonoff, J. S. (1993). The relative importance of bias and variability in the estimation of the variance of a statistic. Journal of the Royal Statistical Society D 42(1), 3-7.

White, H. (1980). A heteroskedasticity-consistent covariance matrix estimator and a direct test for heteroskedasticity. Econometrica 48, 817-838. 


\title{
Appendix A - Size simulation
}

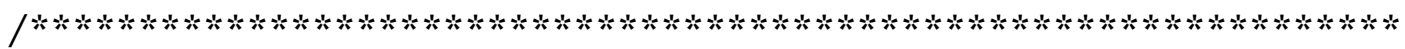 \\ DESCRIPTION: Monte Carlo simulation of quasi-t and $z$ tests under \\ heteroskedasticity. The choice of sample sizes are: 50, 100, 150, \\ 200. Multiple regression. Covariates generated from $|N(\theta, 1)|$ and \\ errors: normal and chi-square with $2,5,10$ degrees of freedom. \\ AUTHOR: Inara Francoyse de Souza Pereira
}

LAST REVISION: October 01,2017

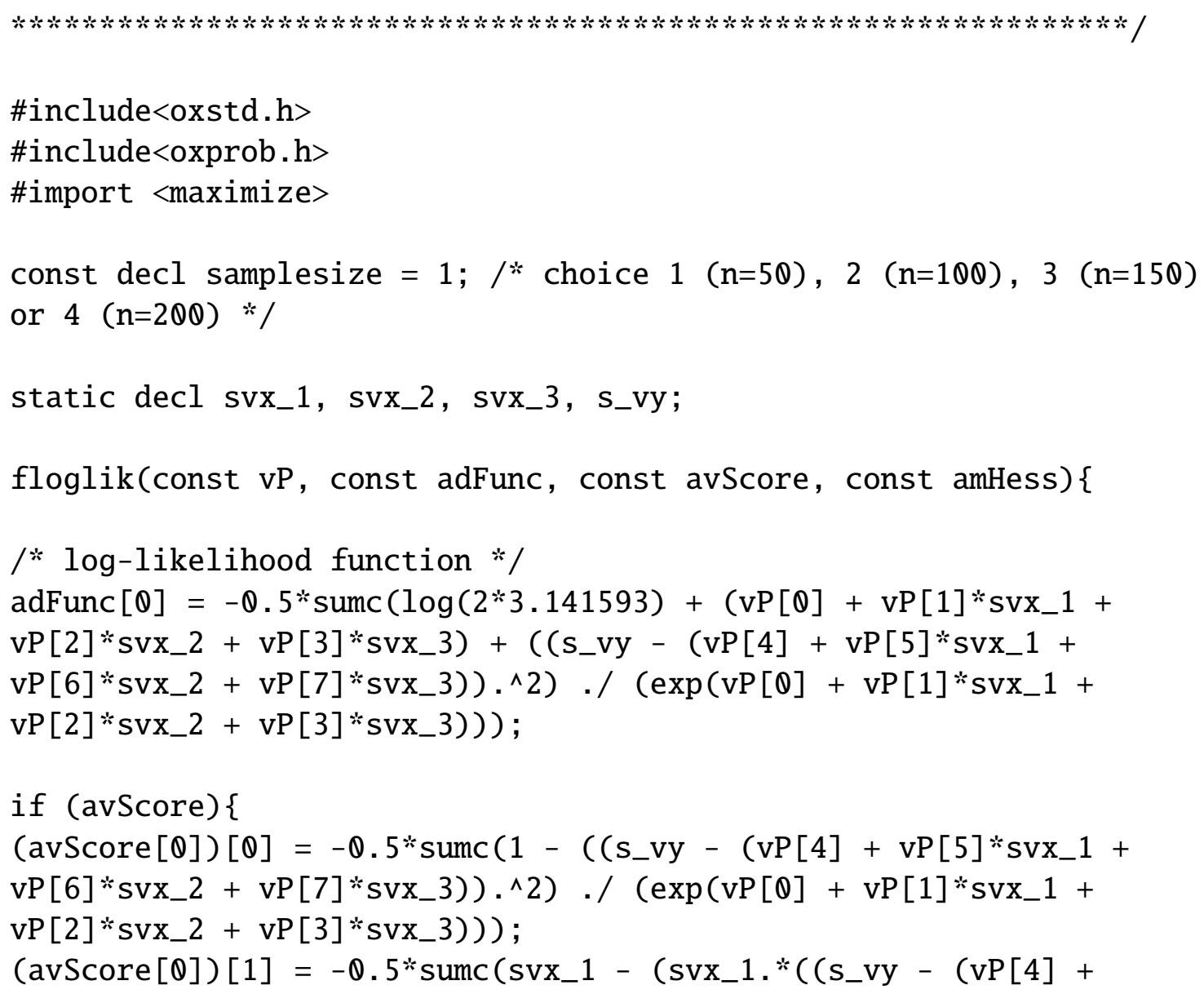




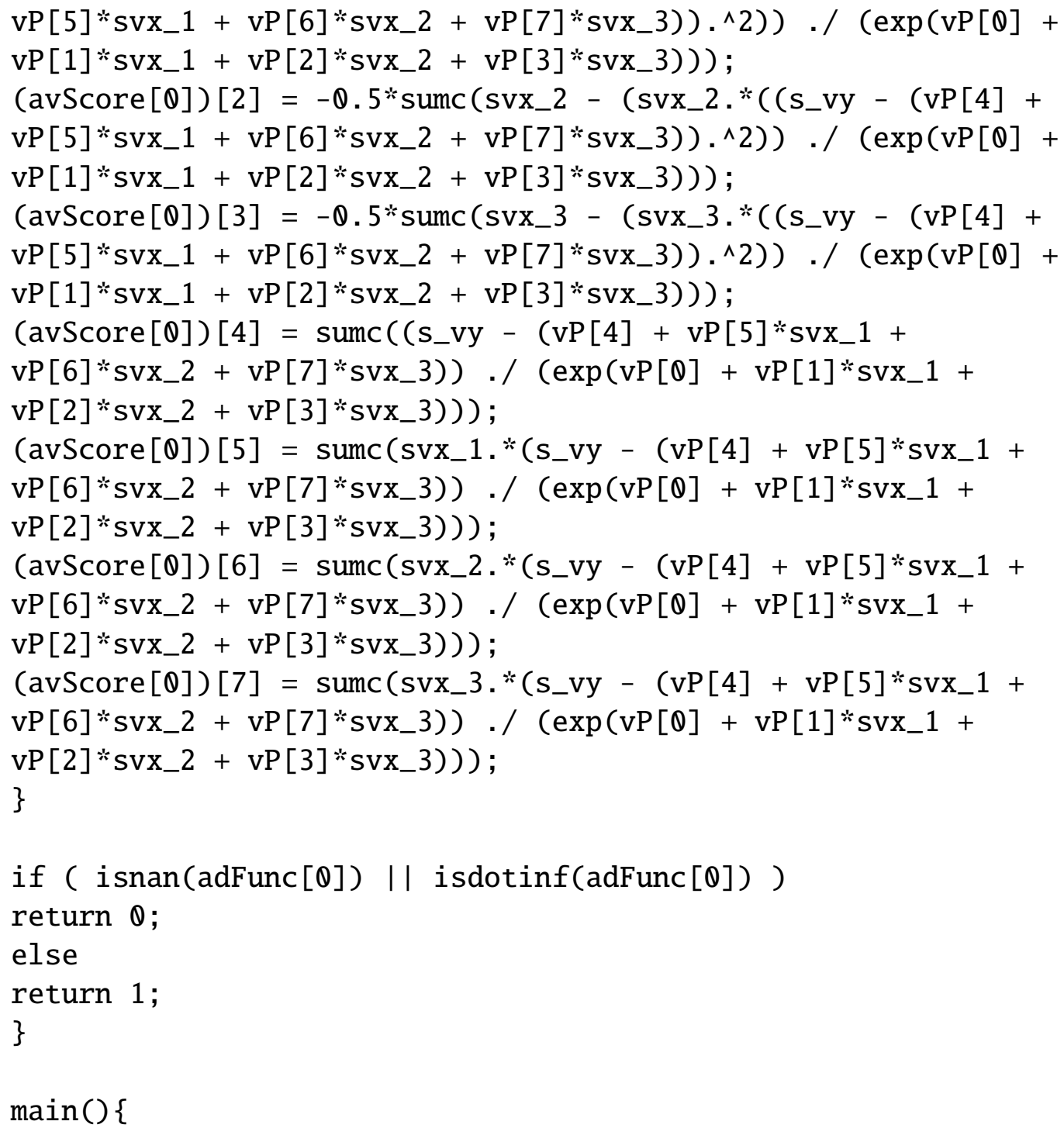
matrixW, vp, dfunc, ir, nrep, temp, eta, exectime, cfailure, ztest, cvz1, cvz5, cvz10, alphachapeu, phi, covm3, covm2, What, betahat2, ztest2, result, count, $\mathrm{HCO}, \mathrm{HC} 2, \mathrm{HC} 3, \mathrm{HC} 4$, ztest3, ztest4, ztest5, teste, ztest7, betahathat,si2, weights, Ww, yw, Xw, olsnumerator, Pt, matrixtemp, hmean, g4m1, g4m2, g4m, weight4m, HC4m, ztest6, I, I2, S, temp4, Psi, temp2, gammatil, temp3, ztest1, cfailure2, betahat3, betaw, counter;

decl nobs $=50 ; / *$ number of base observations (replicate if greater than 50$) * /$

nrep=10000; /* number of Monte Carlo simulations */ 


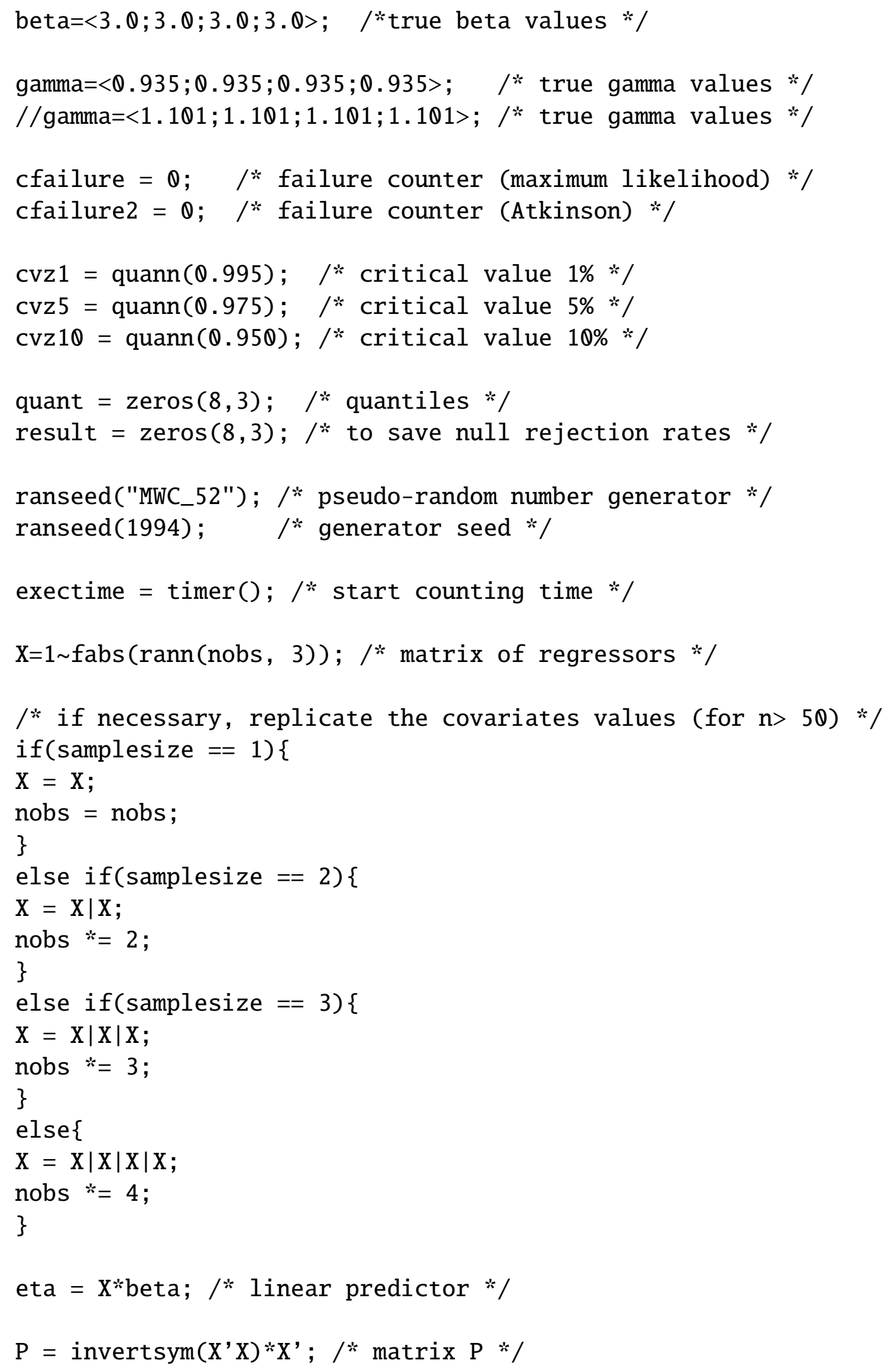




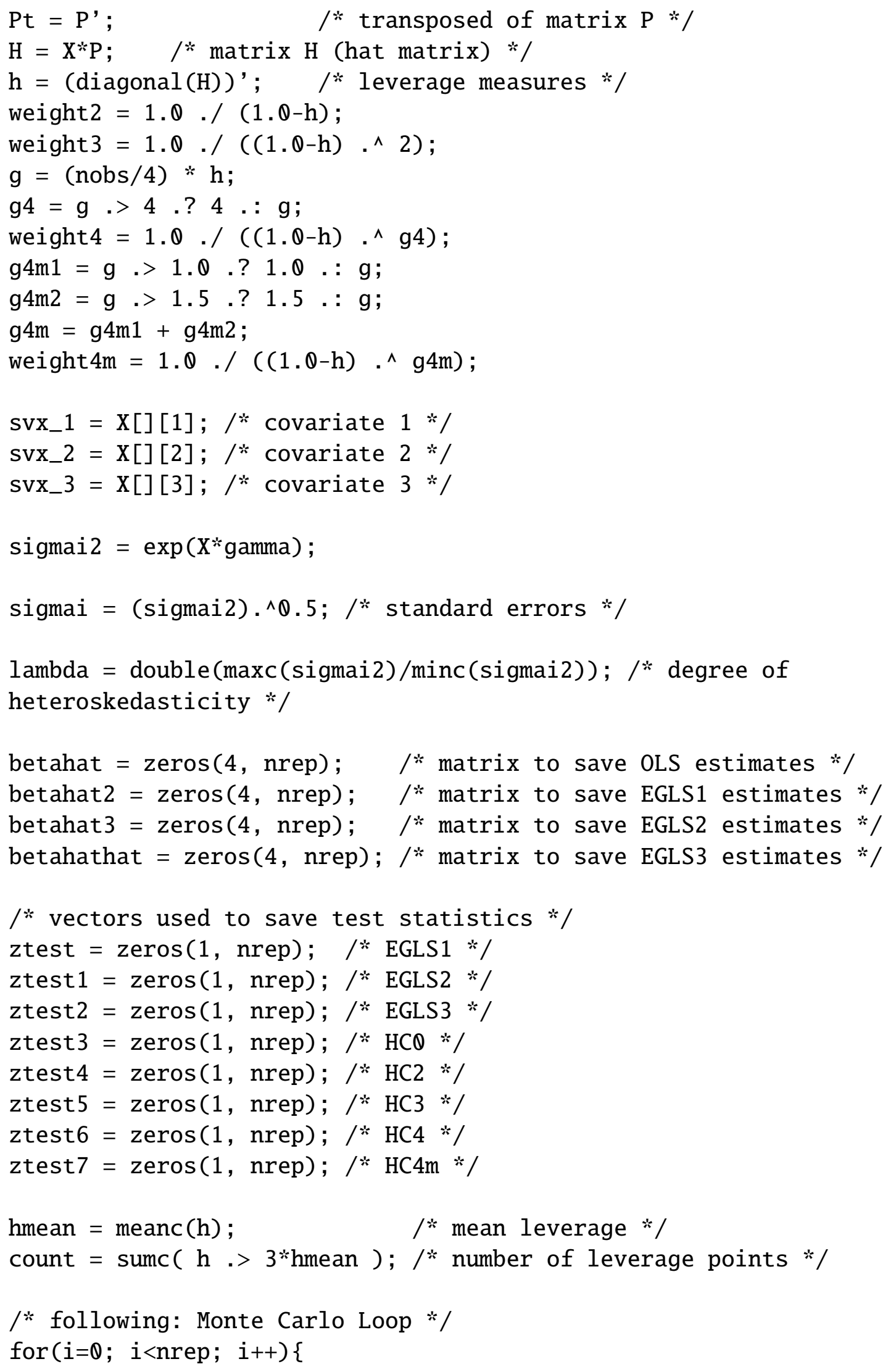




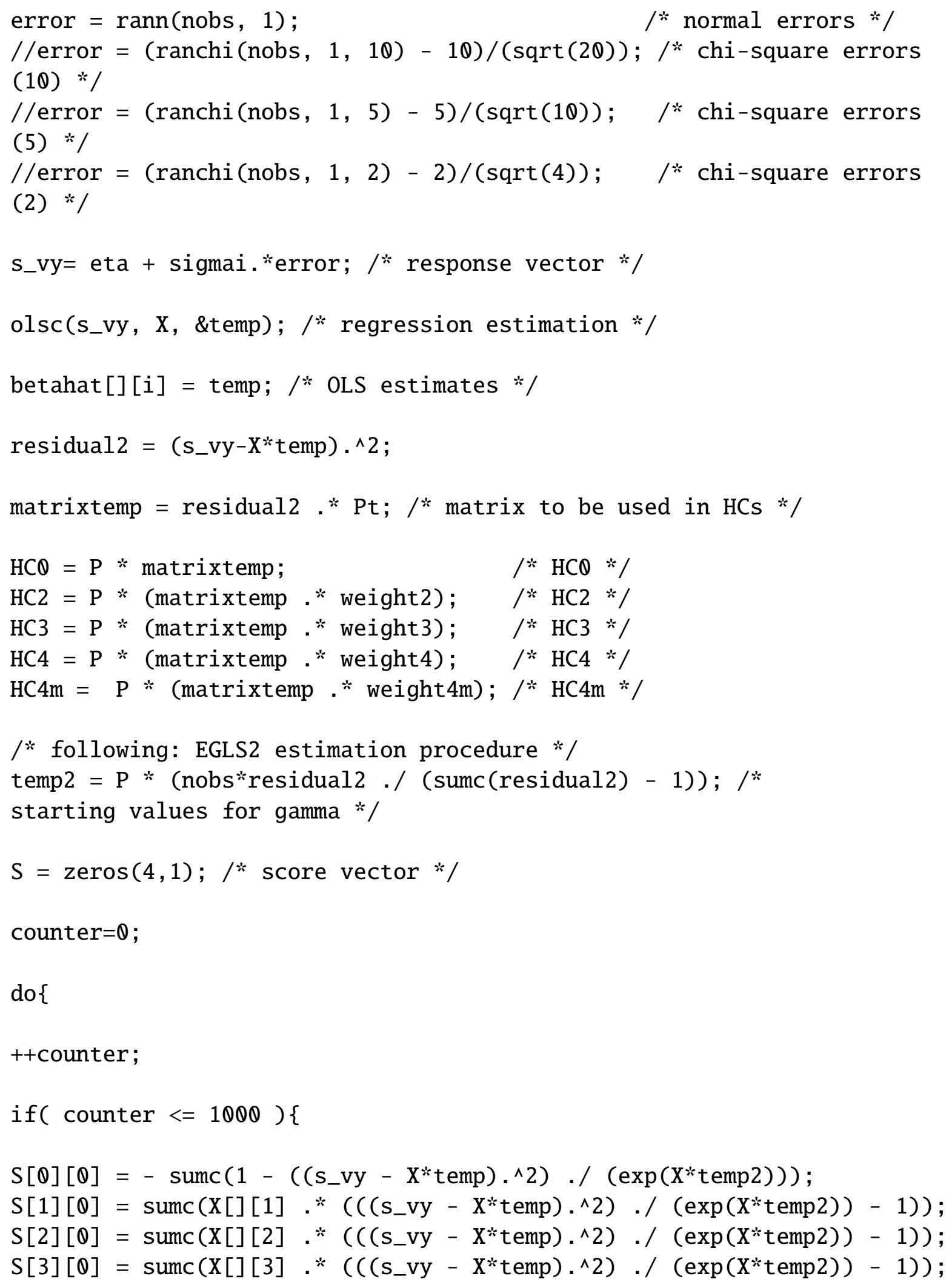




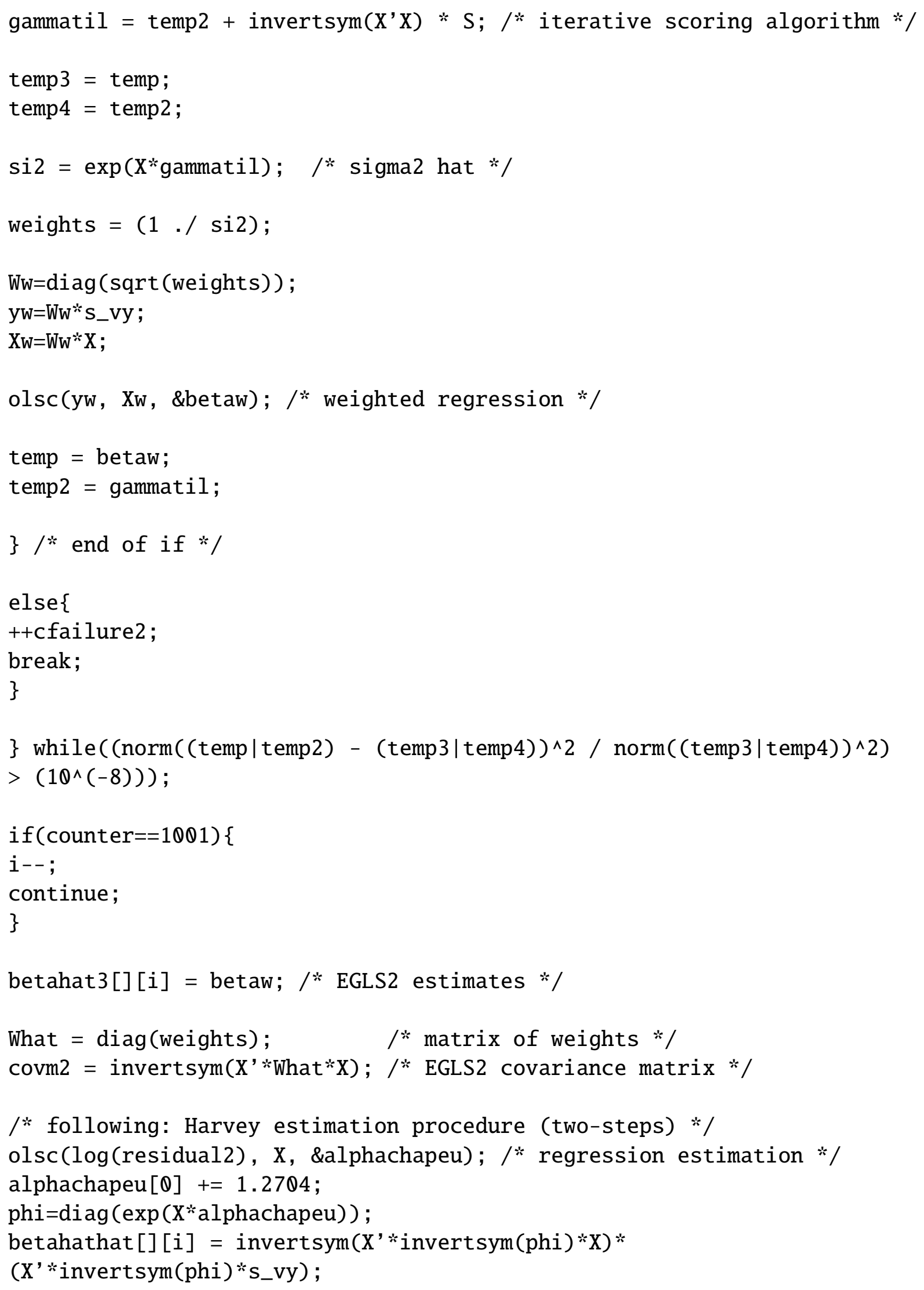


covm3 $=$ invertsym $(\mathrm{X} *$ invertsym $(\mathrm{phi}) * \mathrm{X}) ; / *$ EGLS3 covariance matrix $* /$

$I=\operatorname{zeros}(8,8) ; / *$ inverse of Fisher's information matrix */

/* following: Harvey estimation procedure (maximum likelihood) */

vp $=\langle 5.0 ; 5.0 ; 5.0 ; 5.0 ; 5.0 ; 5.0 ; 5.0 ; 5.0\rangle ; / *$ starting values $* /$

ir $=\operatorname{MaxBFGS}(f \log l i k, \& v p$, dfunc, $\mathbb{0}$, FALSE); /* BFGS with analytical gradient */

$/ *$ convergence check */

if $($ ir $==$ MAX_CONV || ir $==$ MAX_WEAK_CONV ) \{

$I[\theta][\theta]=\operatorname{sumc}\left(1 . / \exp \left(\operatorname{vp}[\theta]+\operatorname{vp}[1] * \operatorname{svx} \_1+\operatorname{vp}[2] * \operatorname{svx} \_2+\right.\right.$ $\left.\left.\operatorname{vp}[3] * \operatorname{svx} \_3\right)\right)$;

$I[1][\theta]=I[\theta][1]=\operatorname{sumc}\left(\operatorname{svx} \_1 . / \exp \left(\operatorname{vp}[\theta]+v p[1] * \operatorname{svx}_{-} 1+\right.\right.$ $\left.\left.\operatorname{vp}[2] * \operatorname{svx} \_2+\operatorname{vp}[3] * \operatorname{svx}_{-} 3\right)\right)$;

$\mathrm{I}[2][\theta]=\mathrm{I}[\theta][2]=\operatorname{sumc}\left(\operatorname{svx} \_2 . / \exp \left(\operatorname{vp}[\theta]+\operatorname{vp}[1] * \operatorname{svx} \_1+\right.\right.$ $\left.\left.\operatorname{vp}[2] * s v x \_2+\operatorname{vp}[3] * \operatorname{svx} \_3\right)\right)$;

$\mathrm{I}[3][\theta]=\mathrm{I}[\mathrm{\theta}][3]=\operatorname{sumc}\left(\operatorname{svx} \_3 . / \exp \left(\operatorname{vp}[\theta]+\mathrm{vp}[1] * \operatorname{svx}_{-} 1+\right.\right.$ $\left.\left.\operatorname{vp}[2] * s v x \_2+\operatorname{vp}[3] * \operatorname{svx} \_3\right)\right)$;

$\mathrm{I}[4][0]=\mathrm{I}[\mathrm{\theta}][4]=\mathrm{I}[5][0]=\mathrm{I}[\mathrm{\theta}][5]=\mathrm{I}[6][0]=\mathrm{I}[\mathrm{\theta}][6]=$

$\mathrm{I}[7][0]=\mathrm{I}[\mathrm{\theta}][7]=\mathrm{I}[4][1]=\mathrm{I}[1][4]=\mathrm{I}[5][1]=\mathrm{I}[1][5]=$

$\mathrm{I}[6][1]=\mathrm{I}[1][6]=\mathrm{I}[7][1]=\mathrm{I}[1][7]=\mathrm{I}[4][2]=\mathrm{I}[2][4]=$

$\mathrm{I}[5][2]=\mathrm{I}[2][5]=\mathrm{I}[6][2]=\mathrm{I}[2][6]=\mathrm{I}[7][2]=\mathrm{I}[2][7]=$

$I[4][3]=I[3][4]=I[5][3]=I[3][5]=I[6][3]=I[3][6]=$

$\mathrm{I}[7][3]=\mathrm{I}[3][7]=0$;

$I[1][1]=\operatorname{sumc}\left(\left(\operatorname{svx} \_1 \cdot^{\wedge} 2\right) . / \exp \left(\operatorname{vp}[\theta]+\operatorname{vp}[1] * \operatorname{svx} \_1+\right.\right.$ $\left.\left.\operatorname{vp}[2] * s v x \_2+\operatorname{vp}[3] * \operatorname{svx} \_3\right)\right)$;

$\mathrm{I}[2][1]=\mathrm{I}[1][2]=\operatorname{sumc}\left(\left(\operatorname{svx} \_1 . * \operatorname{svx} \_2\right) . / \exp (\operatorname{vp}[\theta]+\right.$ $\left.\left.\operatorname{vp}[1] * \operatorname{svx} \_1+\operatorname{vp}[2] * \operatorname{svx} \_2+\operatorname{vp}[3] * \operatorname{svx} \_3\right)\right) ;$

$\mathrm{I}[3][1]=\mathrm{I}[1][3]=\operatorname{sumc}\left(\left(\operatorname{svx}{ }_{-} 1 * \operatorname{svx}_{-} 3\right) . / \exp (\operatorname{vp}[0]+\right.$ $\left.\left.\operatorname{vp}[1] * \operatorname{svx} \_1+\operatorname{vp}[2] * \operatorname{svx} \_2+\operatorname{vp}[3] * \operatorname{svx} \_3\right)\right) ;$

$\mathrm{I}[2][2]=\operatorname{sumc}\left(\left(\operatorname{svx} \_2 \cdot{ }^{\wedge} 2\right) . / \exp \left(\operatorname{vp}[\theta]+\operatorname{vp}[1]^{*} \operatorname{svx} \_1+\right.\right.$ $\left.\left.\operatorname{vp}[2] * \operatorname{svx} \_2+\operatorname{vp}[3] * \operatorname{svx} \_3\right)\right)$;

$\mathrm{I}[3][3]=\operatorname{sumc}\left(\left(\operatorname{svx}{ }^{3} 3 \cdot{ }^{\wedge} 2\right) \cdot / \exp \left(\operatorname{vp}[\theta]+\operatorname{vp}[1] * \operatorname{svx}_{-} 1+\right.\right.$ $\operatorname{vp}[2] *$ svx_2 + vp[3]*svx_3));

$\mathrm{I}[3][2]=\mathrm{I}[2][3]=\operatorname{sumc}\left(\left(\operatorname{svx} \_2 . * \operatorname{svx} \_3\right) . / \exp (v p[0]+\right.$ $\left.\left.\operatorname{vp}[1] * \operatorname{svx} \_1+\operatorname{vp}[2] * \operatorname{svx} \_2+\operatorname{vp}[3] * \operatorname{svx}_{-} 3\right)\right)$;

$\mathrm{I}[4][4]=$ nobs $/ 2$;

$\mathrm{I}[5][5]=0.5 * \operatorname{sumc}\left(\operatorname{svx} \_1 . \wedge 2\right)$;

$I[5][4]=I[4][5]=0.5 * \operatorname{sumc}\left(\operatorname{svx} \_1\right)$;

$I[6][4]=I[4][6]=0.5 * \operatorname{sumc}\left(\operatorname{svx} \_2\right)$;

$I[7][4]=I[4][7]=0.5 * \operatorname{sumc}\left(\operatorname{svx} \_3\right)$;

$\mathrm{I}[6][6]=0.5 * \operatorname{sumc}\left(\operatorname{svx} \_2 .^{\wedge} 2\right)$; 


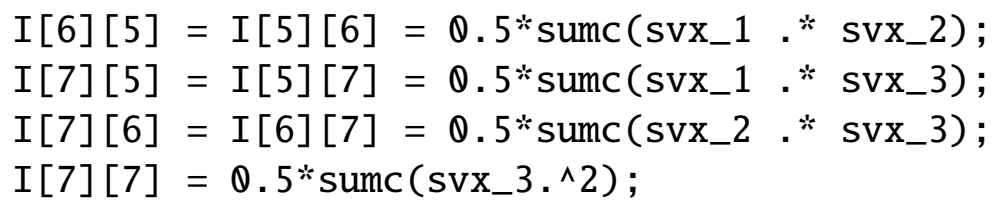




$$
\begin{aligned}
& \text { result }[2][1]=\operatorname{sumr}(\text { fabs }(z \operatorname{ztest} 2[0][]) .>\text { cvz5)/nrep*100;/* EGLS3 } 5 \% * / \\
& \text { result [3][1] = sumr (fabs (ztest3[0][]) .> cvz5)/nrep*100; } / * \text { HCQ } 5 \% * / \\
& \text { result }[4][1]=\operatorname{sumr}(\text { fabs }(z t e s t 4[0][]) .>\text { cvz5) } / \text { nrep*100; } / * \text { HC2 } 5 \% * / \\
& \text { result }[5][1]=\operatorname{sumr}(\text { fabs (ztest5[0][]) .> cvz5)/nrep*100; } / * \text { HC3 } 5 \% * / \\
& \text { result [6] [1] }=\operatorname{sumr}(\text { fabs }(z \operatorname{test6}[0][]) .>\text { cvz5) } / \mathrm{nrep} * 100 ; / * \text { HC4 } 5 \% * / \\
& \text { result [7][1] }=\operatorname{sumr}(\text { fabs (ztest7[0][]) .> cvz5)/nrep*100;/* HC4m 5\%*/ } \\
& \text { result }[0][2]=\operatorname{sumr}(\text { fabs }(z \operatorname{zest}[0][]) .>\operatorname{cvz} 10) / \mathrm{nrep} * 100 ; \quad / * \operatorname{EGLS} 110 \% * / \\
& \text { result }[1][2]=\operatorname{sumr}(\text { fabs }(z t e s t 1[0][]) .>\operatorname{cvz} 10) / \text { nrep*100; } / * \text { EGLS2 } 10 \% * / \\
& \text { result }[2][2]=\operatorname{sumr}(\text { fabs }(z \operatorname{test} 2[0][]) .>\text { cvz10)/nrep*100; /* EGLS3 } 10 \% * / \\
& \text { result [3] [2] = sumr }(\text { fabs (ztest3[0][]) .> cvz10)/nrep*100;/* HCO } 10 \% * / \\
& \text { result }[4][2]=\operatorname{sumr}(\text { fabs }(z t e s t 4[0][]) .>\operatorname{cvz} 10) / \text { nrep }^{*} 100 ; / * \text { HC2 } 10 \% * / \\
& \text { result [5][2] }=\operatorname{sumr}(\text { fabs }(z \operatorname{test5}[0][]) .>\operatorname{cvz} 10) / \text { nrep } * 100 ; / * \text { HC3 } 10 \% * / \\
& \text { result [6] [2] }=\operatorname{sumr}(\text { fabs (ztest6[0][]) .> cvz10)/nrep*100; /* HC4 } 10 \% * / \\
& \text { result [7][2] }=\operatorname{sumr}(\text { fabs }(z \operatorname{test} 7[0][]) .>\operatorname{cvz} 10) / \mathrm{nrep} * 100 ; / * \mathrm{HC} 4 \mathrm{~m} 10 \% * / \\
& / * \text { The quantiles of the different test statistics are calculated } \\
& \text { and used as exact critical values in the power simulations */ }
\end{aligned}
$$

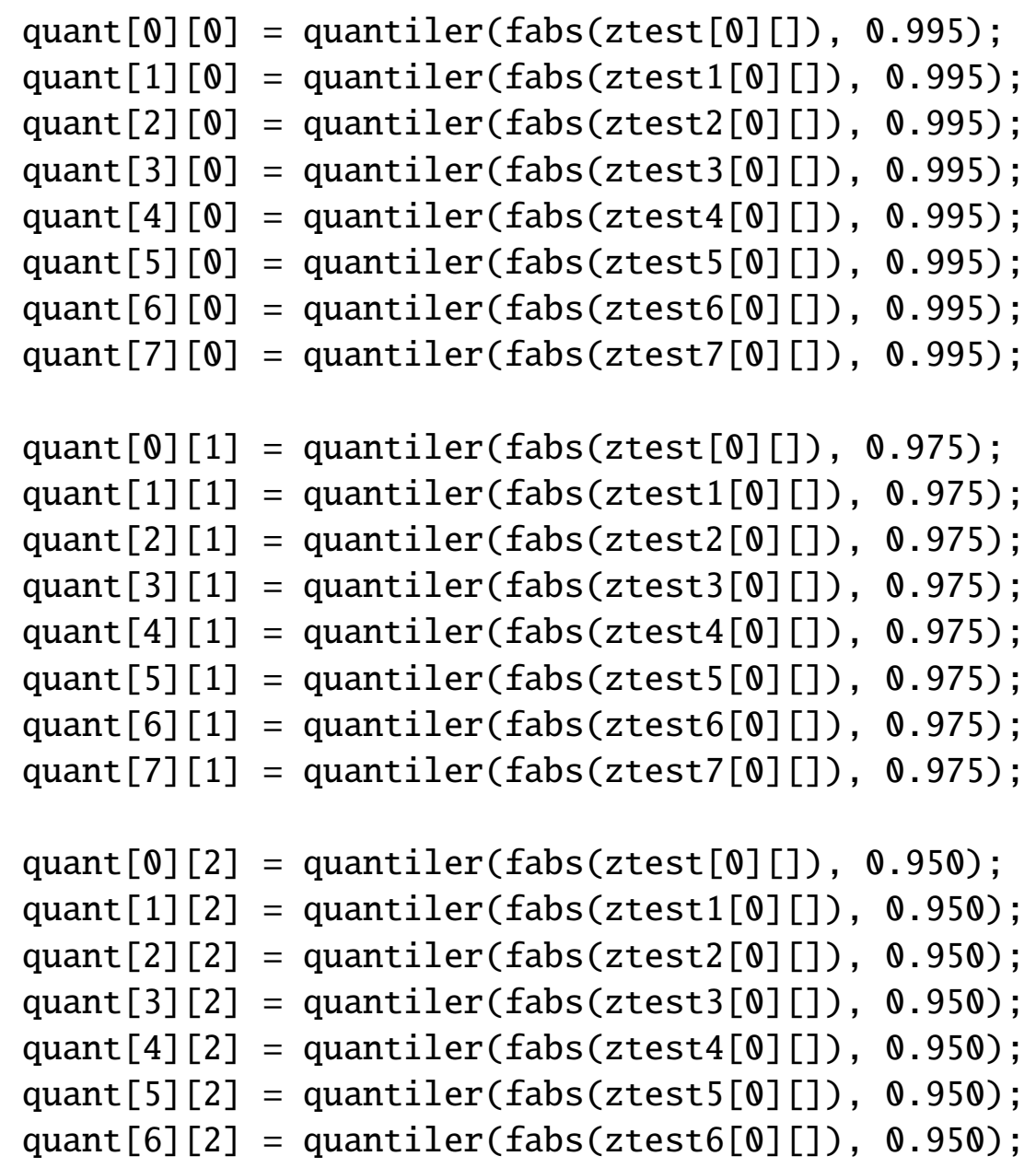




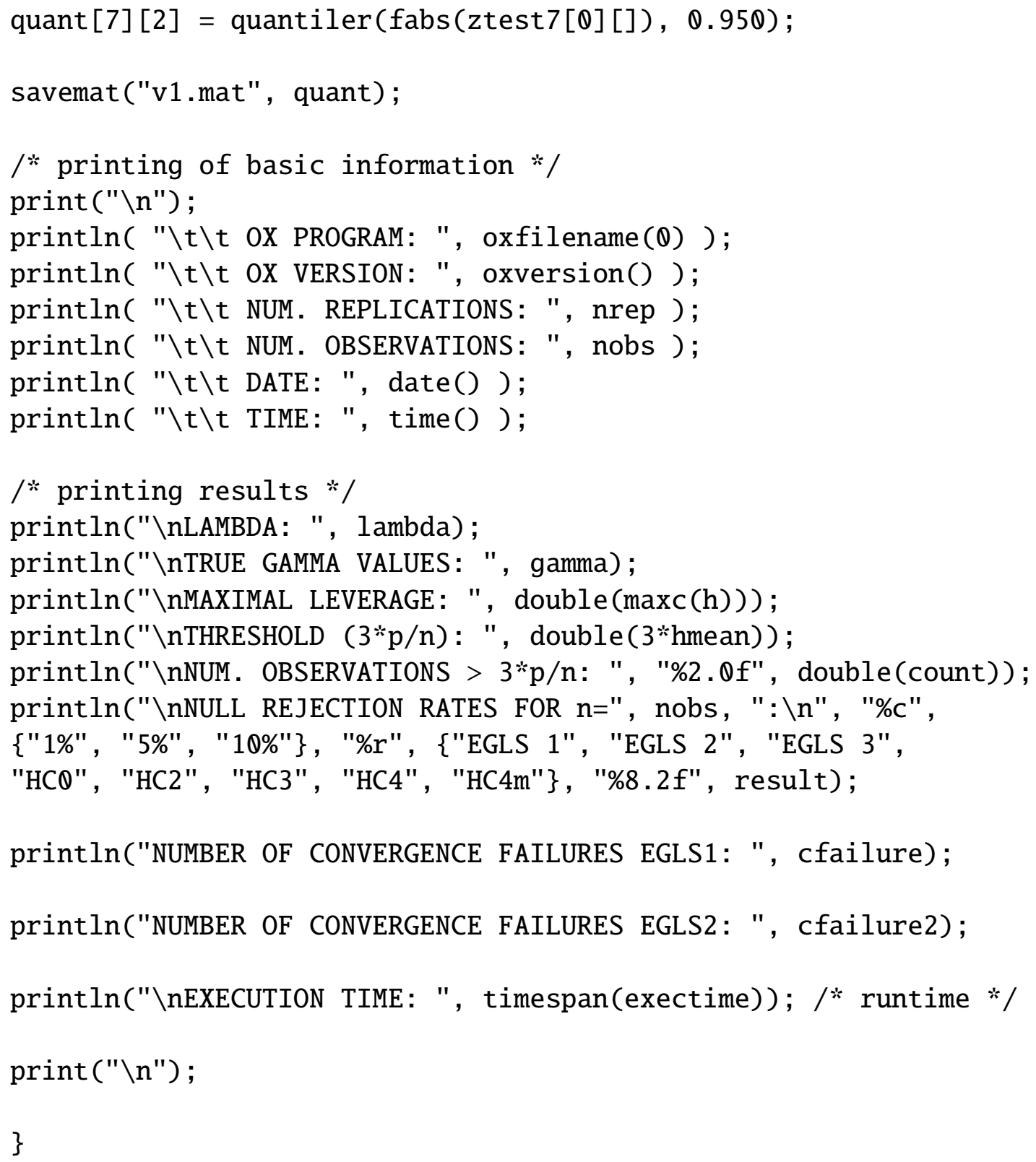




\title{
Appendix B - Power simulation
}

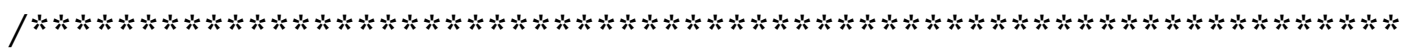 \\ DESCRIPTION: Monte Carlo simulation of quasi-t and $z$ tests under \\ heteroskedasticity. The choice of sample sizes are: 50, 100, 150, \\ 200. Multiple regression. Covariates generated from $|N(\theta, 1)|$ and \\ errors: normal and chi-square with 2, 5, 10 degrees of freedom. \\ AUTHOR: Inara Francoyse de Souza Pereira
}

LAST REVISION: October 01,2017

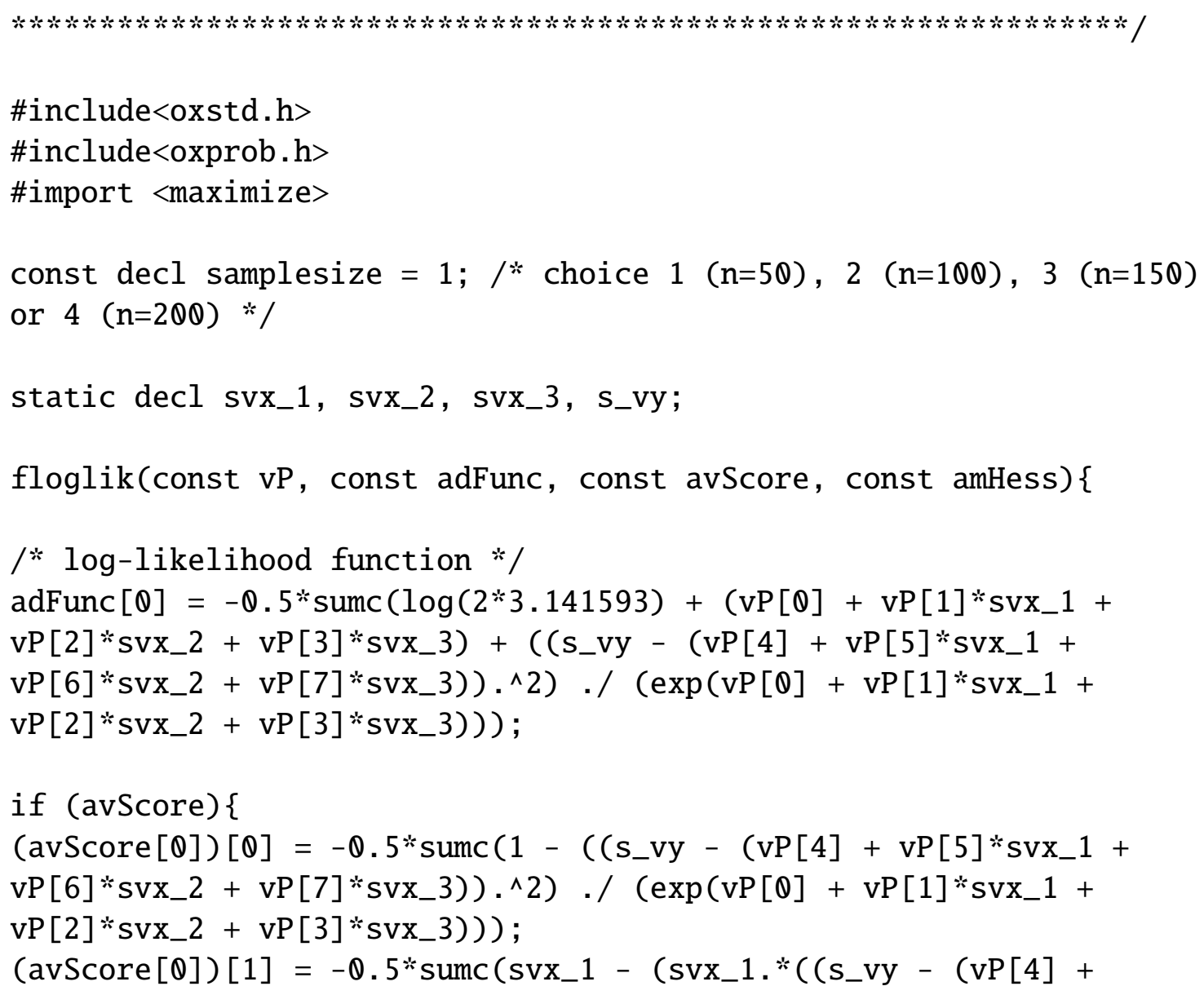




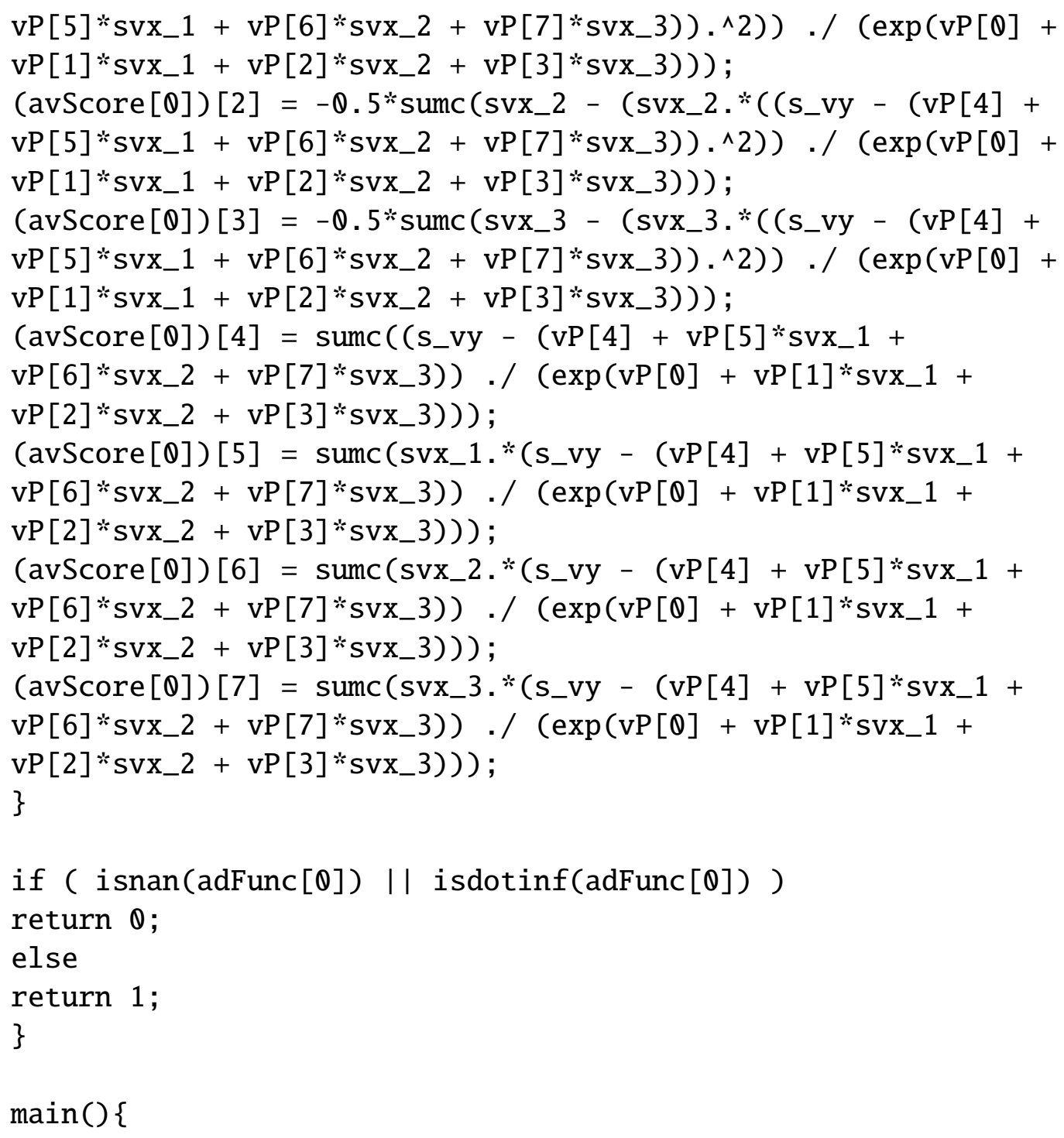
cvz5, cvz10, alphachapeu, phi, covm3, covm2, What, betahat2, ztest2, result, count, $\mathrm{HCO}, \mathrm{HC} 2, \mathrm{HC} 3$, HC4, ztest3, ztest4, ztest5, ztest7, betahathat, si2, weights, $\mathrm{Ww}, \mathrm{yw}, \mathrm{Xw}$, olsnumerator, Pt, matrixtemp, hmean, g4m1, g4m2, g4m, weight4m, HC4m, ztest6, I, I2, S, temp4, Psi, temp2, gammatil, temp3, ztest1, cfailure2, betahat3, betaw, mat1, mat2, mat3, mat4, mat5, mat6, mat7, mat8, mat9, mat10, mat11, mat12, mat13, mat14, mat15, mat16, mat17, mat18, mat19, mat20, mat21, mat22, mat23, mat24, mat25, mat26, mat27, mat28, mat29, mat30, mat31, mat32;

/* quantiles obtained in the size simulations */ 


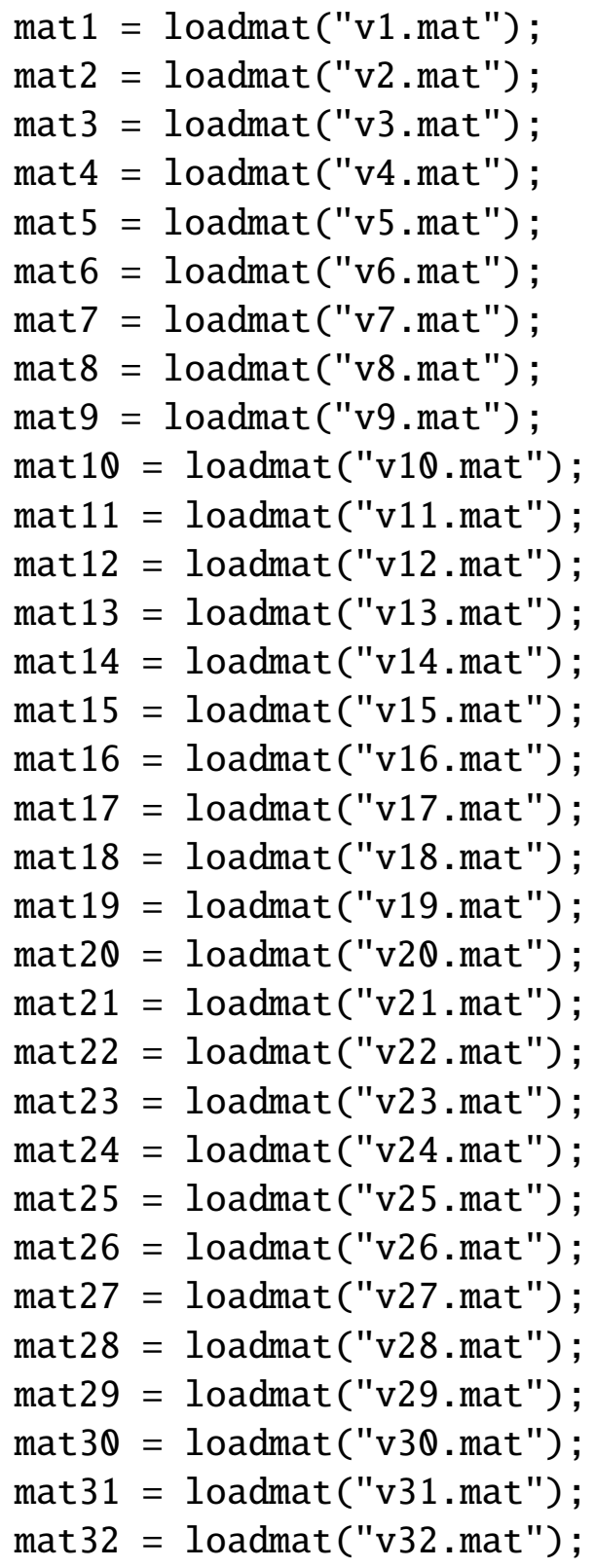

decl nobs $=50 ; / *$ number of base observations (replicate if greater than 50 ) */

nrep=10000; /* number of Monte Carlo simulations */

beta $=\langle 3.0 ; 3.0 ; 6.0 ; 3.0>; / *$ true beta values $* /$

gamma $=<0.935 ; 0.935 ; 0.935 ; 0.935>; \quad / *$ true gamma values $* /$ $/ /$ gamma $=<1.101 ; 1.101 ; 1.101 ; 1.101>; / *$ true gamma values */

cfailure $=0 ; \quad / *$ failure counter (maximum likelihood) */ 


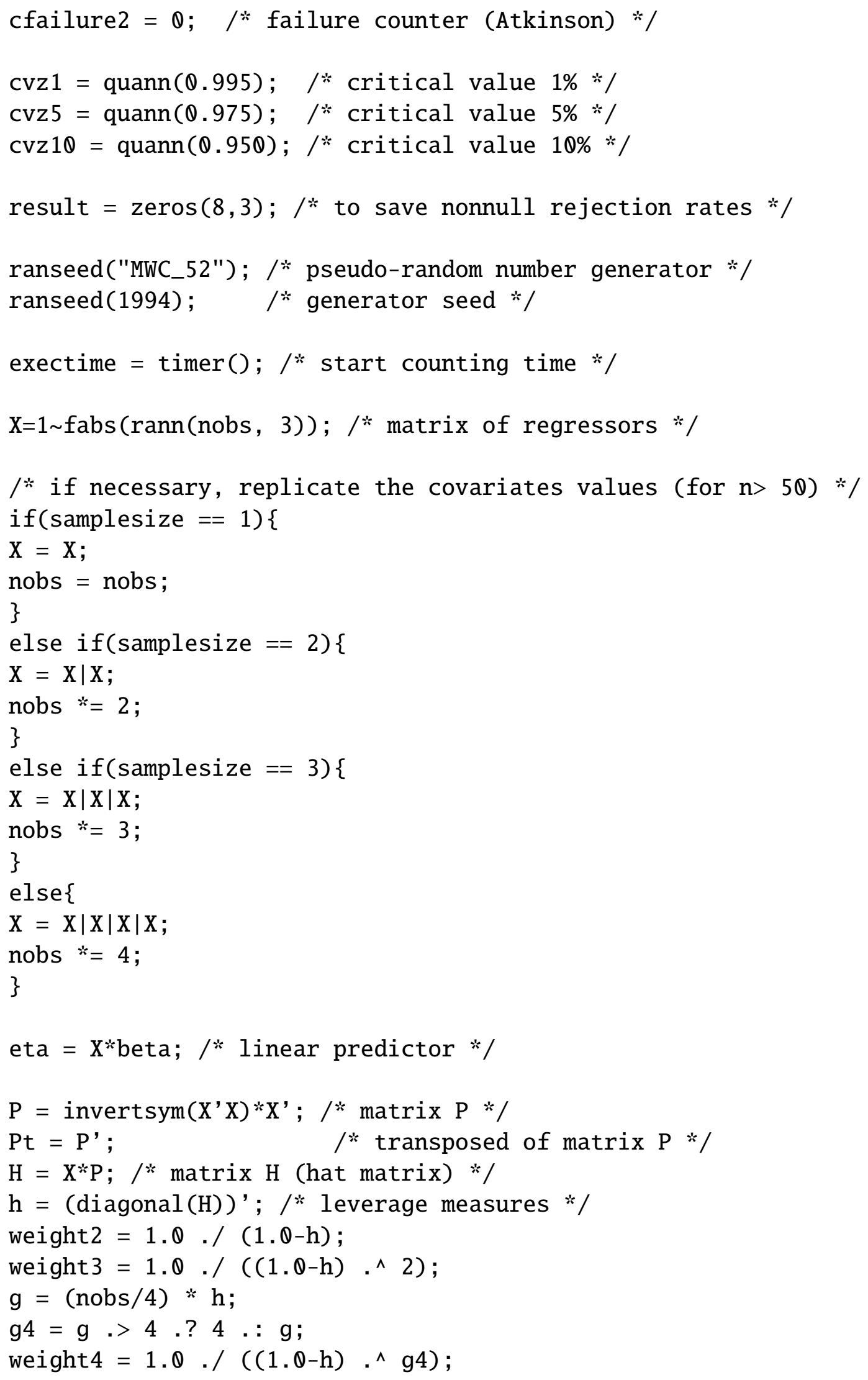




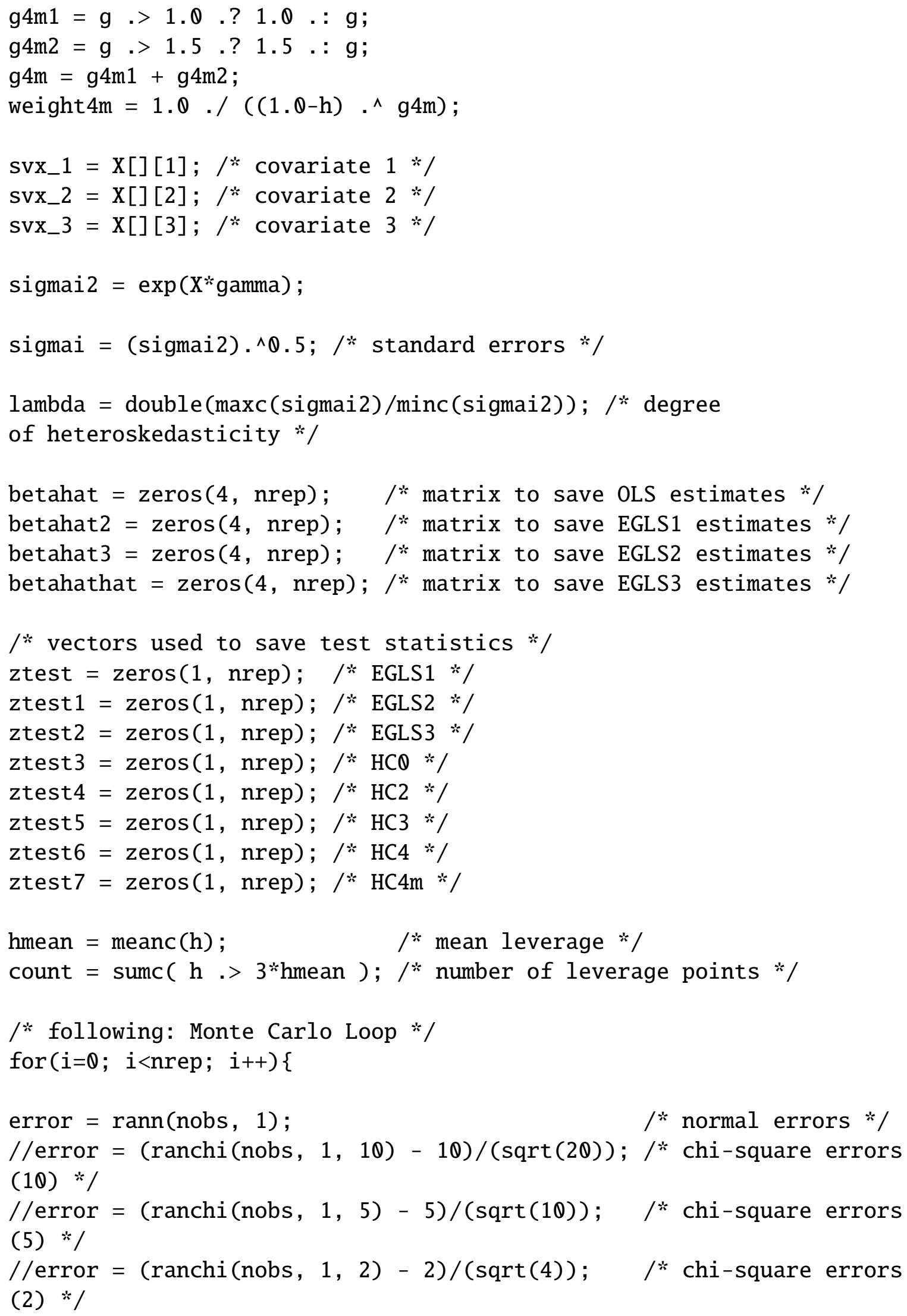




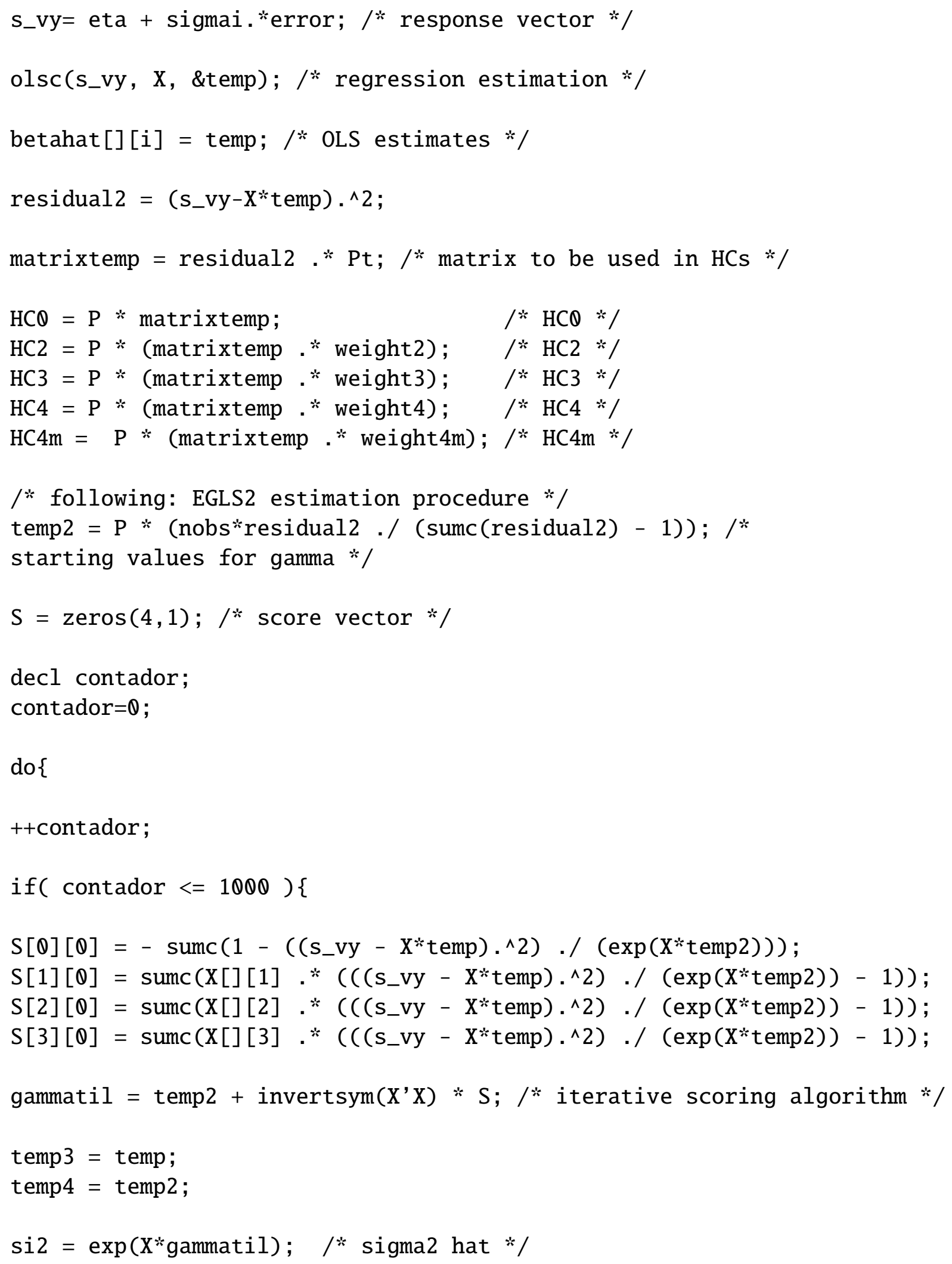




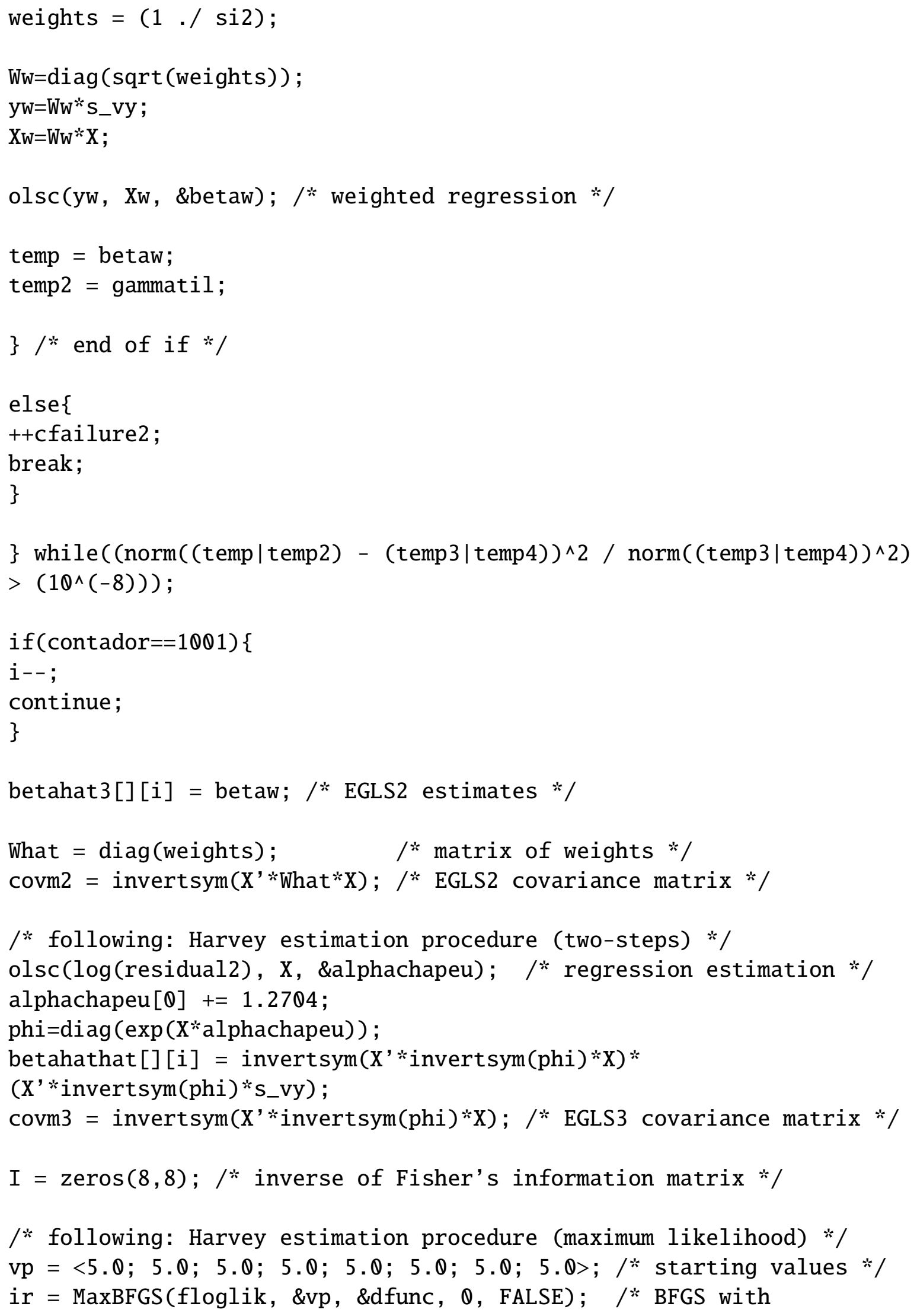




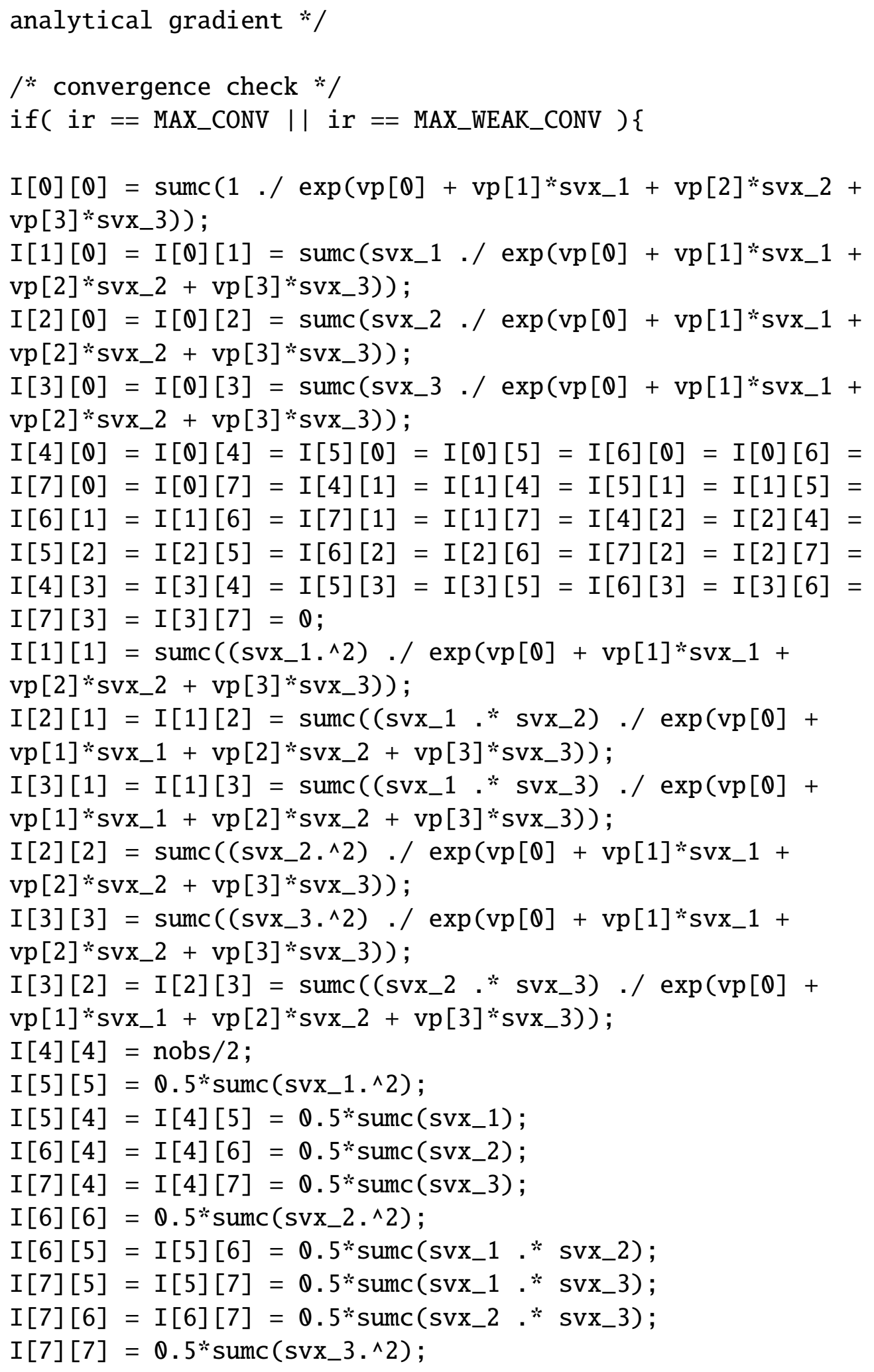




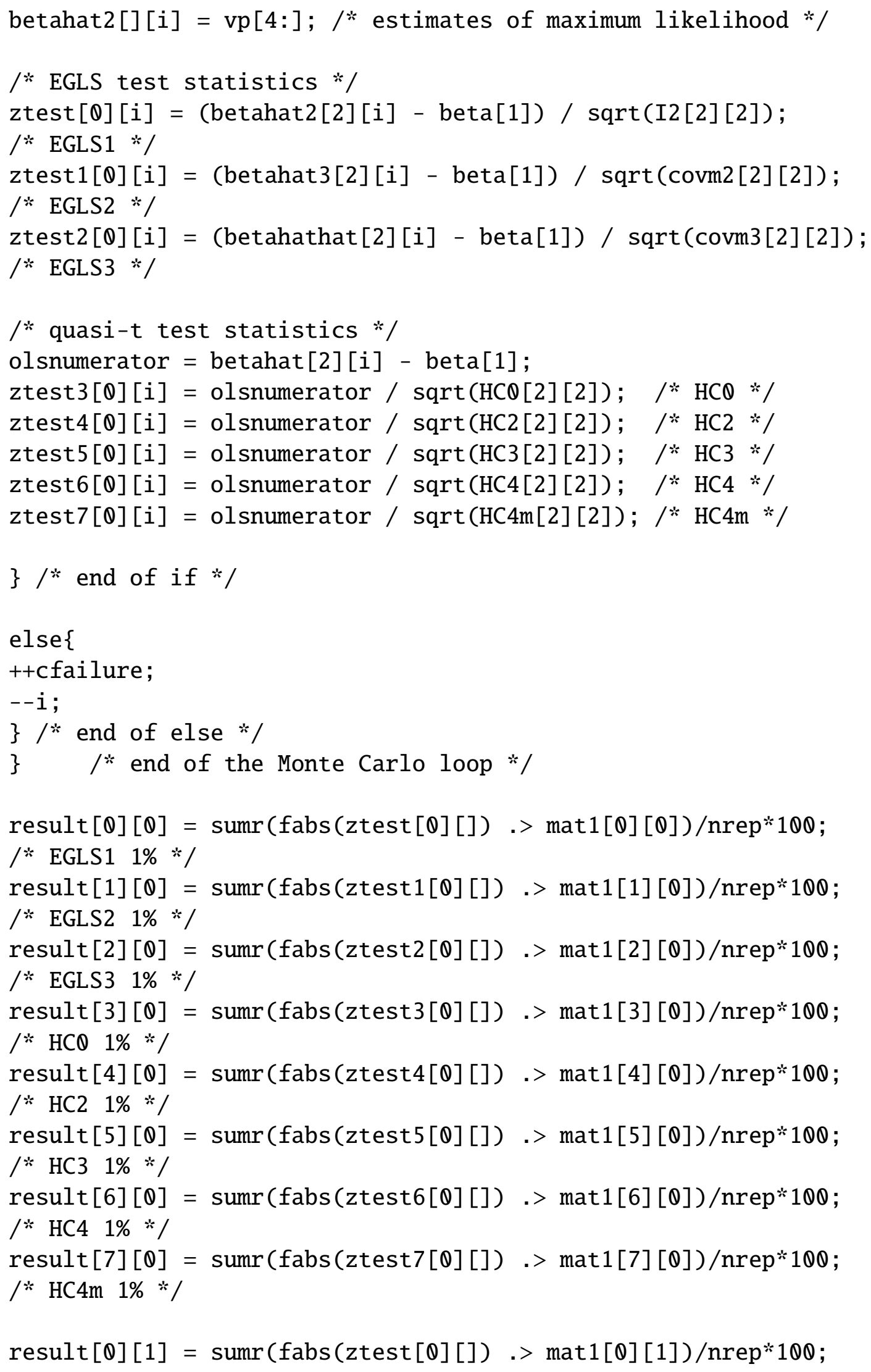




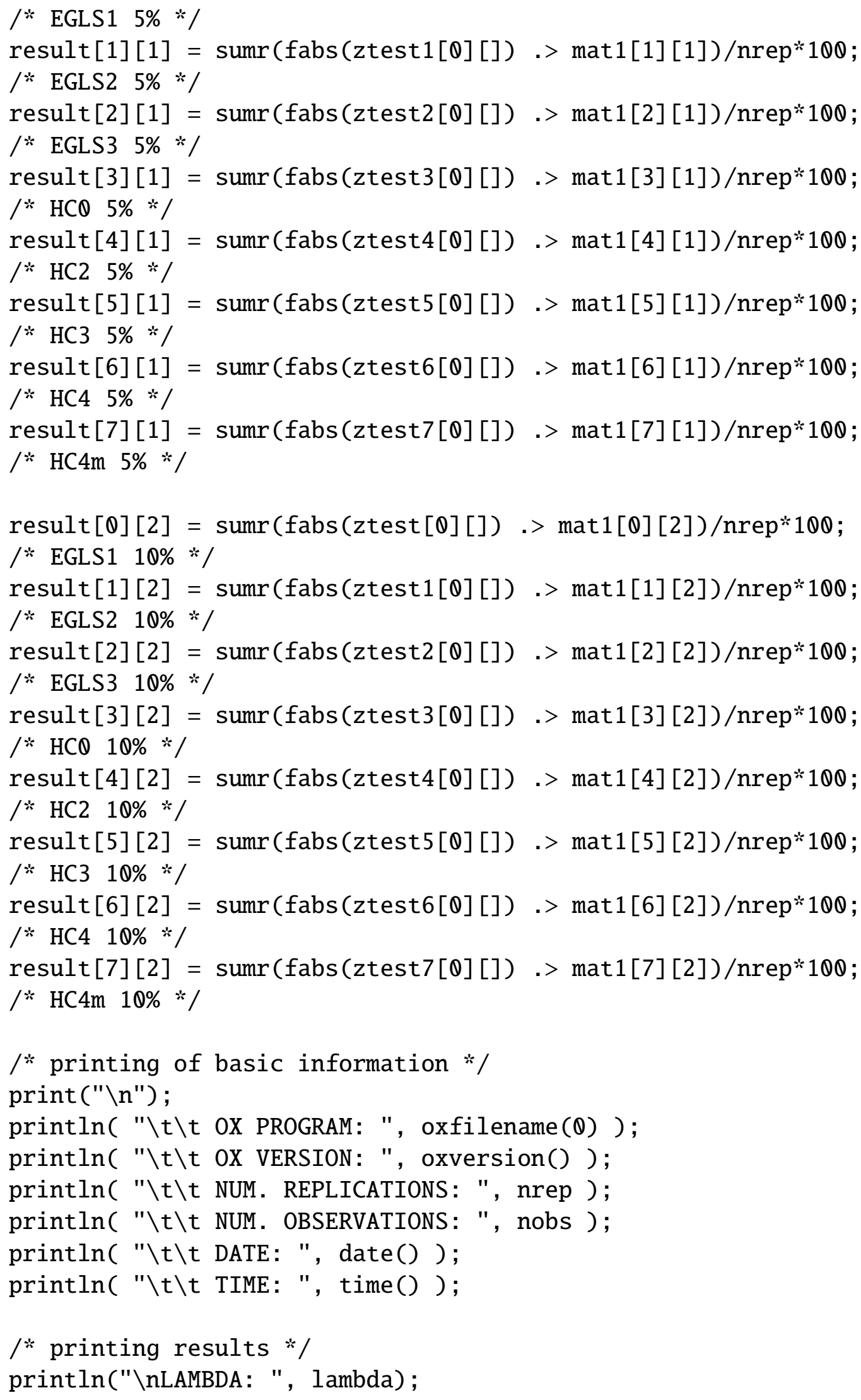


println("\nTRUE GAMMA VALUES: ", gamma);

println("\nMAXIMAL LEVERAGE: ", $\operatorname{double}(\operatorname{maxc}(\mathrm{h}))$ );

println("\nTHRESHOLD (3*p/n): ", double(3*hmean));

println("\nNUM. OBSERVATIONS > 3*p/n: ", "\%2.of", double(count));

println("\nNONNULL REJECTION RATES FOR $n="$, nobs, ": $\backslash n$ ", "\%c", \{"1\%", "5\%", "10\%"\}, "\%r", \{"EGLS 1", "EGLS 2", "EGLS 3", "HCO", "HC2", "HC3", "HC4", "HC4m"\}, "\%8.2f", result);

println("NUMBER OF CONVERGENCE FAILURES EGLS1: ", cfailure);

println("NUMBER OF CONVERGENCE FAILURES EGLS2: ", cfailure2);

println("\nEXECUTION TIME: ", timespan(exectime)); /* runtime */

print ("\n") ;

\} 